\title{
ELEMENTARY STUDENTS' UNDERSTANDING OF FRACTIONS WITH DYNAMIC TECHNOLOGY
}

\author{
A Dissertation \\ presented to \\ the Faculty of the Graduate School \\ at the University of Missouri-Columbia
}

In Partial Fulfillment

of the Requirements for the Degree

Doctor of Philosophy

by

SHEUNGHYUN YEO

Dr. Corey Webel, Dissertation Advisor

JULY 2020 
(C) Copyright by Sheunghyun Yeo 2020

All Rights Reserved 
The undersigned, appointed by the dean of the Graduate School, have examined the dissertation entitled

\section{ELEMENTARY STUDENTS’ UNDERSTANDING OF FRACTIONS WITH DYNAMIC TECHNOLOGY}

presented by Sheunghyun Yeo, a candidate for the degree of doctor of philosophy, and hereby certify that, in their opinion, it is worthy of acceptance.

Dr. Corey Webel, Committee Chair

Dr. Susan Empson, Committee Member

Dr. James Tarr, Committee Member

Dr. Samuel Otten, Committee Member

Dr. Isa Janke, Committee Member 


\section{ACKNOWLEDGEMENTS}

My heart is full of gratitude in acknowledging the people who make this dissertation possible. First of all, I would like to thank 30 students who volunteered to participate in this study and 10 students who participated in the pilot study. Without sharing your creative and brilliant thinking and precious time with me, this study would not have taken place. When you expressed joy and engagement in the study, it was a delightful and rewarding moment during this journey.

I would like to thank my advisor, Dr. Corey Webel, for his incredible and unlimited supports of me and my work. Over the past four years, he has been shaped my academic identity and honed my skills. He is a great mentor who listens to and challenges my ideas, which has helped me to refine my thinking about research. His insight, wisdom, expertise, passion, and encouragement allowed me to pursue my research interest, mathematics education and technology. I also have been fortunate to be his first advisee and to collaborate with him for the NSF-funded STEAM project. And I loved to hang out with Melissa, Emma Grace, Harrison, and Elliot.

I would like to thank Dr. Susan Empson who provided support throughout this study. She guided my exploration of the measurement approach to fractions, which provided an intersection with the use of technology. Her perspective and feedback on my work helped me refine my ideas and framing and improve the quality of my work. I had learned more about your work when translating your book into Korean, "Extending Children's Mathematics", which made me enter children's mathematical thinking.

I would like to thank Dr. James Tarr who helped me make a successful journey during the doctoral program. At every critical stage, he provided guidance and advice to 
wisely solve faced problems. Especially, during the job search, he advised all the detail for even small steps to prepare for interviews. I have learned a great deal not only from research meetings but also from our personal interactions. I would definitely miss your sense of humor.

I would like to thank Dr. Samuel Otten who has been a strong supporter and advocate. During the online PD project, 'Two Minute Teacher's Guide', he showed the process of how an interesting research idea could be formalized as doable research. He exposed me to online teaching, which has been essential to current my work, and he still contributes to extend my understanding of mathematical education through Math ED podcast. It was my honor to celebrate the 100th episode by introducing Vygotsky's book.

I would like to thank Dr. Isa Jahnke who inspired me to study educational technology. From her class, I had learned the consistency between teaching philosophy as an instructor and theoretical underpinning as a researcher. And I still wrestle with her questions, "How do students learn?", "How can technology be used meaningfully?" The process of answering those questions vitalized my journey for this study.

Many thanks to the faculty members and the graduate students with whom I have worked and studied: Dr. Kathryn Chval, Dr. John Lannin, Dr. Zandra de Araujo, Dr. Chuck Munter, Dr. Vickie Spain, Dr. Ruby Ellis, Chris E., Ruveyda, Erin, Kimberly, Chris A., Cara, Wenmin, Cris, Amy, Phi, Cassie, Heather, Brendan, Mitchelle, Courtney, Erica, Jessica, and JP. They have contributed to my learning and made the graduate student experience invaluable and memorable.

I would like to thank Mizzou Graduate school to provide academic and financial supports. Especially, It has been honorable to be a recipient of G. Ellsworth Huggins 
Scholarships. This was helpful to fulfill my professional ambition of becoming a faculty member in higher education.

Lastly, I would like to thank my parents, sister, and brother for their sincere dedication and emotional support. I also appreciate my father-in-law and mother-in-law for their endless prayer for my future. Yuna and Gina, I love your every kiss and every hug when I am studying. Both of you are the reason of my life. Last, but not least, Yoonjung, my wife, has always been my strength throughout the times. It would be impossible for me to express my appreciation from your sacrifice and even more impossible for me to thank you for making my dream come true together. 사랑해! 


\section{TABLE OF CONTENTS}

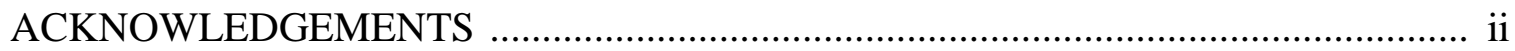

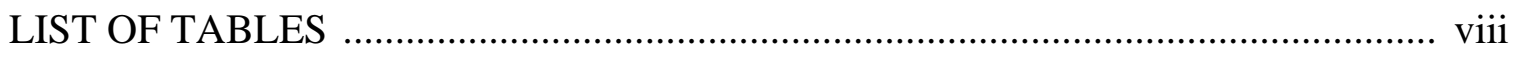

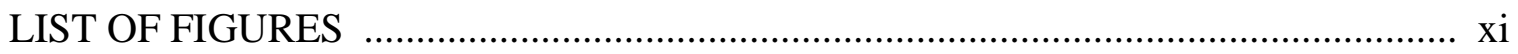

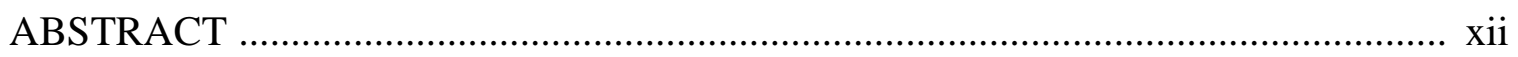

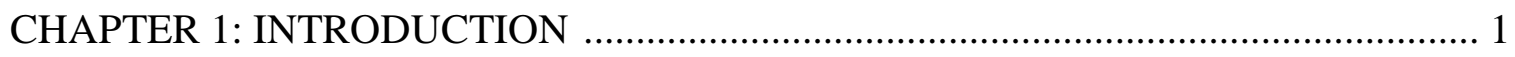

Measurement Approach to Fractions through Linear Representations .......................... 3

The Use of Dynamic Technology to Enhance Mathematical Understanding .............. 7

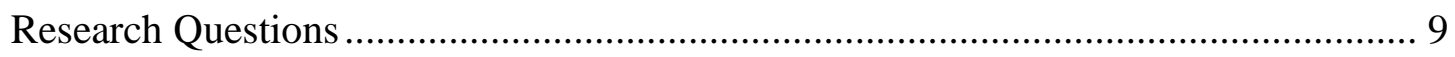

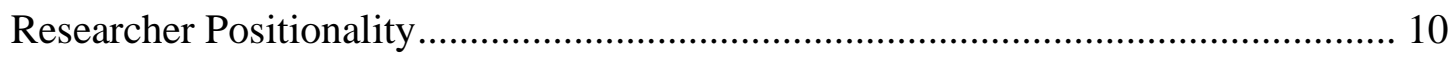

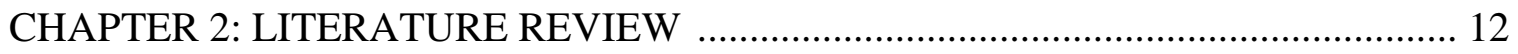

Conceptual Framework .................................................................................. 12

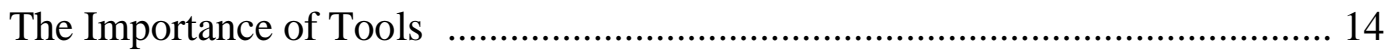

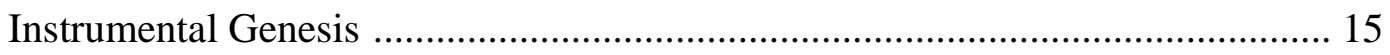

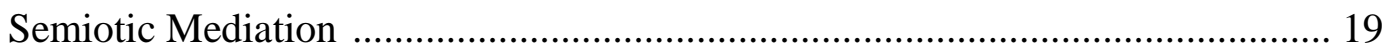

Instrumented Mediation ....................................................................... 22

A Measurement Approach to Fractions ………………………………………..... 24

Challenges and Alternative Approaches for Learning Fractions ….................... 24

Theoretical Learning From a Measurement Approach ...................................... 28

Key Constructs of Fraction as Measure ……………...................................... 32

Dynamic Mathematics and Principles of Dynamism ………..................................... 33

Developing Fraction Concepts With Digital Technology ........................................ 35

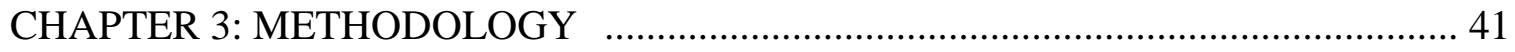




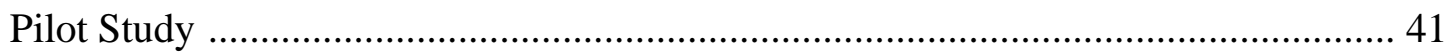

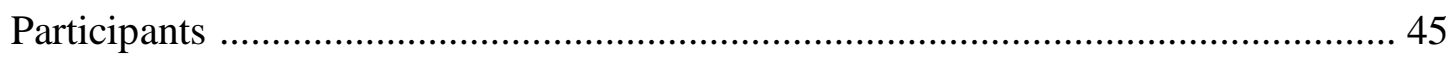

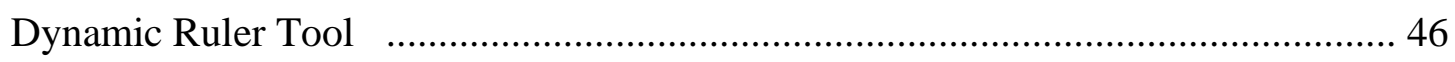

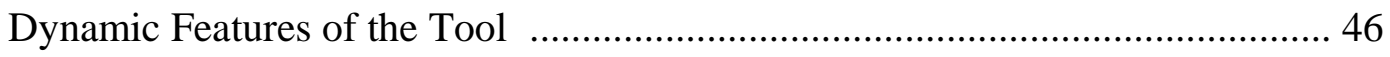

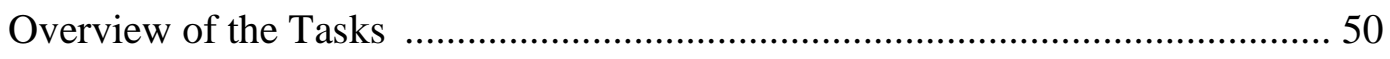

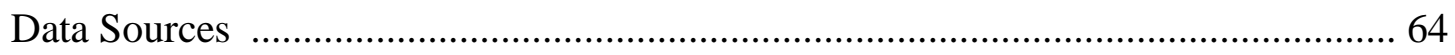

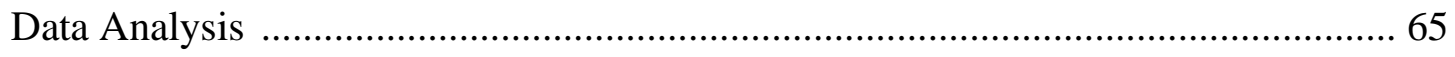

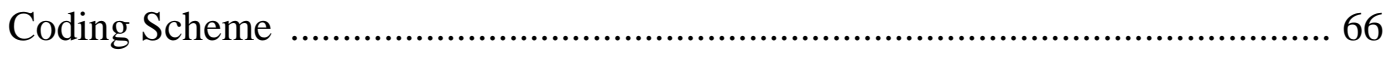

Development of the Strategy Types Framework ………………………............ 80

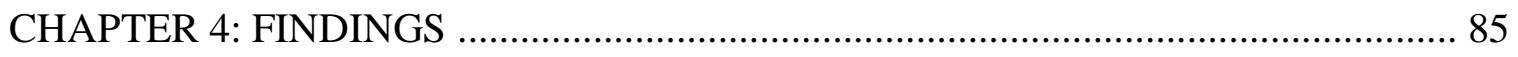

Students' Strategy Types Framework on Fraction as Measure ……........................... 85

Developing a Framework for Students' Strategies ................................................ 86

Fraction as Measure Using the Dynamic Ruler .................................................. 87

Illustration of Strategy types for Fraction as Measure ……………......................... 91

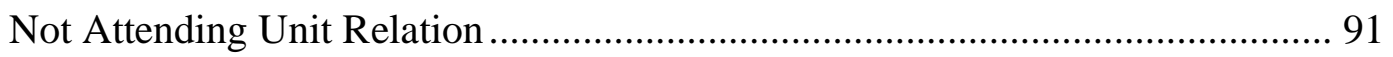

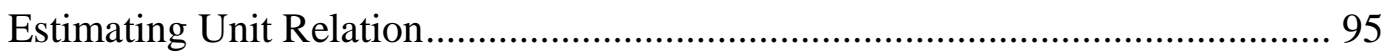

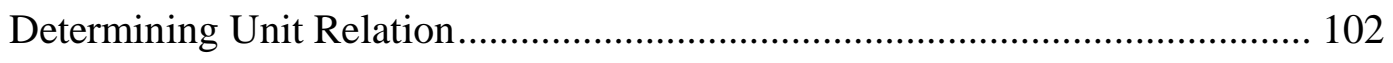

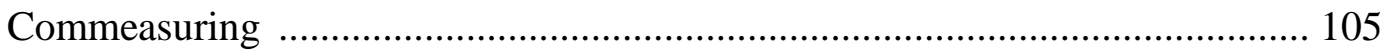

Patterns of Strategy Types Across Fraction-as-Measurement Tasks........................ 113

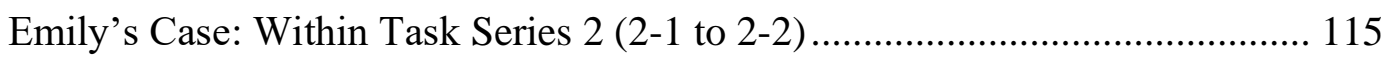

Sewoong's Case: Within Task Series 2 (2-2 to 2-4) ......................................... 123

Fran's Case: Within Task Series 3 (3-1 to 3-2) ................................................. 130

Alex's Case: Within Task Series 3 (3-2 to 3-4) …………............................. 137 
Charim's Case: Across Task Series 2 and 3 (2-4 to 3-1) ................................. 142

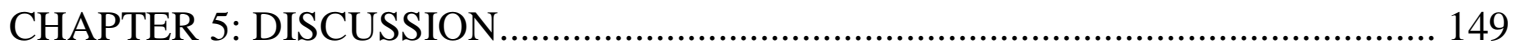

Instrumented Mediation ............................................................................. 150

Actions with Knowledge - Meaning Making ............................................. 152

Affordances and Constraints - Meaning Making ..................................... 153

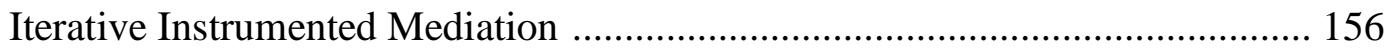

Directions for Future Research …................................................................ 158

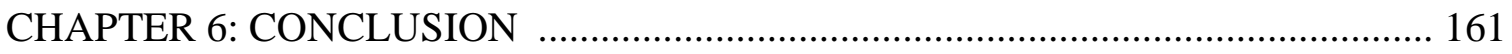

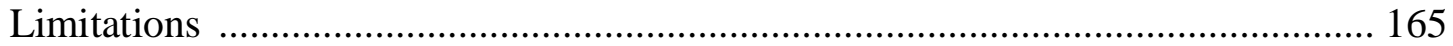

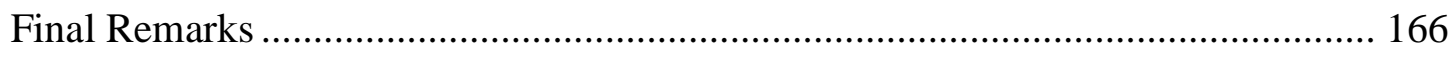

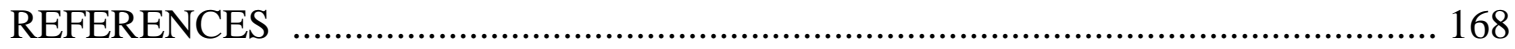

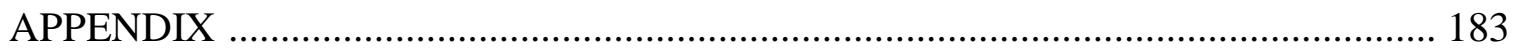

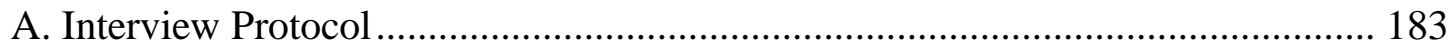

B. Initial Coding Scheme......................................................................... 188

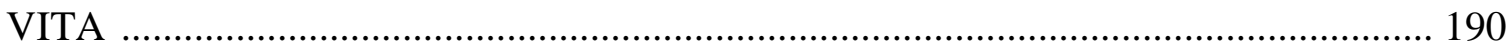




\section{LIST OF TABLES}

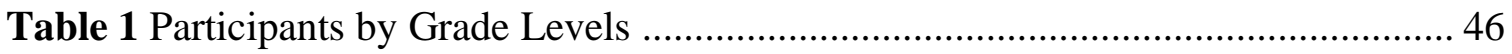

Table 2 Summary of Fraction as Measure Tasks ...................................................... 51

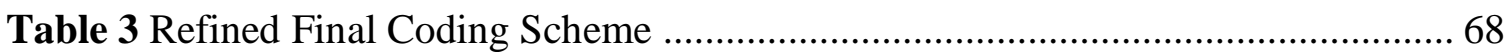

Table 4 Preliminary Framework for Strategy Types of Fraction as Measure ................. 82

Table 5 Types of Children's Strategies for Fraction as Measure Tasks ........................ 86

Table 6 Types of Students' Strategies About Fraction as Measure .............................. 88

Table 7 Distribution of Strategy Types by Tasks and Grade Levels ............................ 90

Table 8 Patterns of Strategy Types by Task Series ................................................... 114 


\section{LIST OF FIGURES}

Figure 1 Measuring Length A With a Single Unit (Left) and B With Two Units (Right) 6

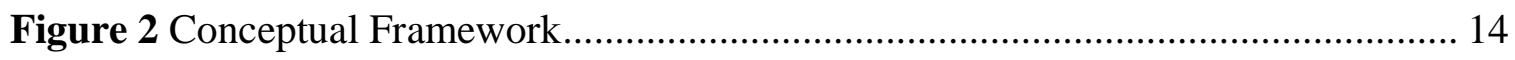

Figure 3 Fraction Hexagonal Task ................................................................... 26

Figure 4 Measuring A With Unit k and a Partial Unit ............................................ 28

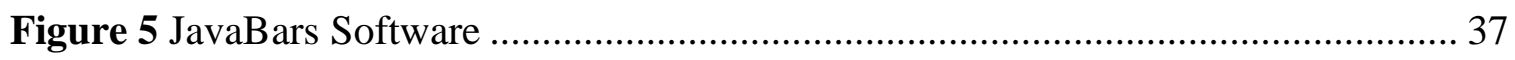

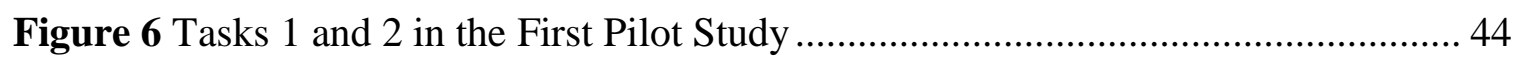

Figure 7 Screenshot of the Tutorial........................................................................ 47

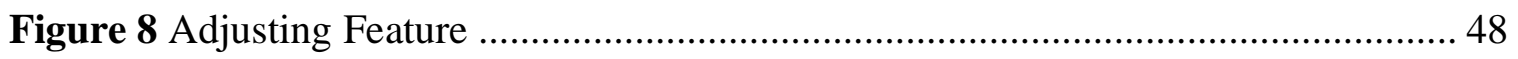

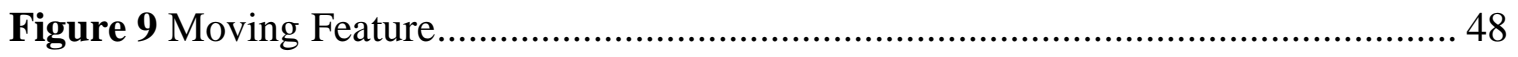

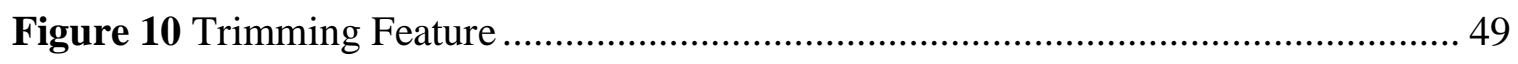

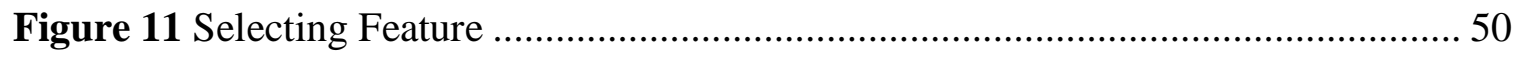

Figure 12 Examples of Task in Series 2 (2-1, 2-3, and 2-4) ..................................... 53

Figure 13 Key Terms in Fraction-as-Measurement Tasks ....................................... 55

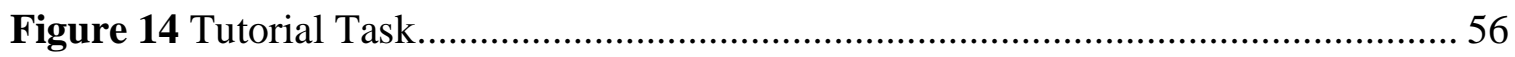

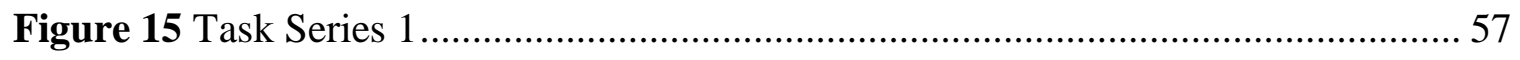

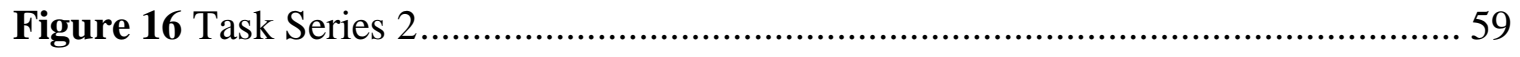

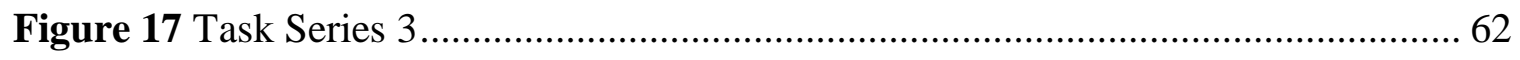

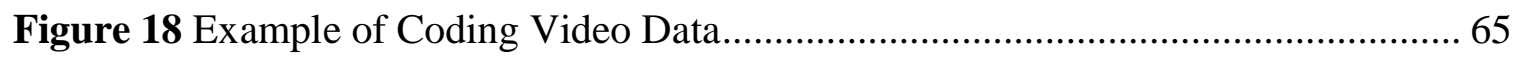

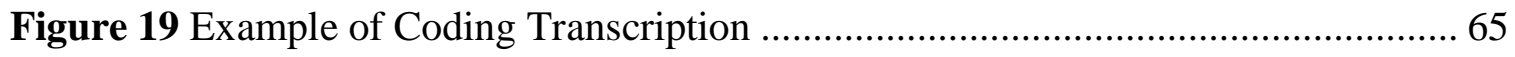

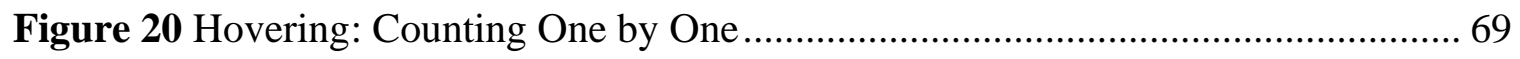

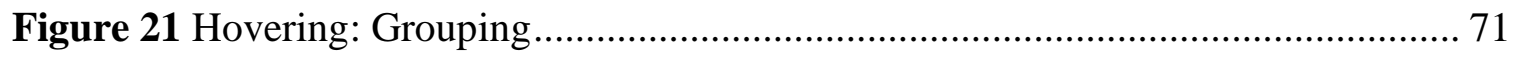

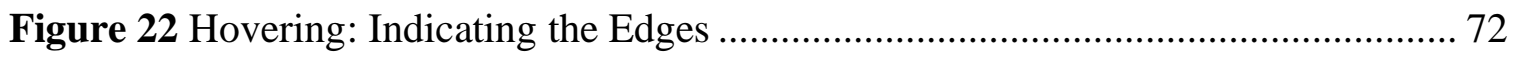




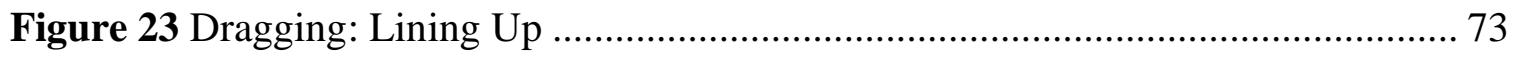

Figure 24 Dragging: Changing Sizes ................................................................... 74

Figure 25 Dragging: Commeasuring....................................................................... 76

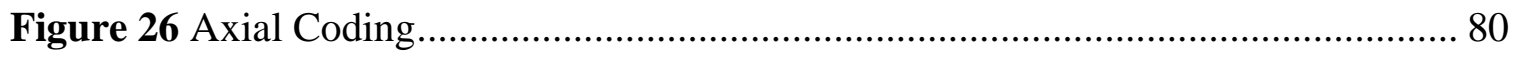

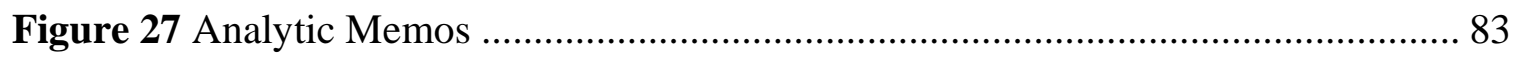

Figure 28 Mary's Use of the Default Size Unit ............................................................. 93

Figure 29 Sungho's Estimation of the Length With a Partial Unit .................................. 94

Figure 30 Lauren's Estimation of the Leftover.............................................................. 96

Figure 31 Eric's Estimation of the Leftover .............................................................. 98

Figure 32 Sumin's Use of an Intermediate Unit .......................................................... 100

Figure 33 Peter's Use of an Intermediate Unit ........................................................... 101

Figure 34 Charim's Use of an Intermediate Unit........................................................ 103

Figure 35 Sewoong's Use of Intermediate Units ...................................................... 104

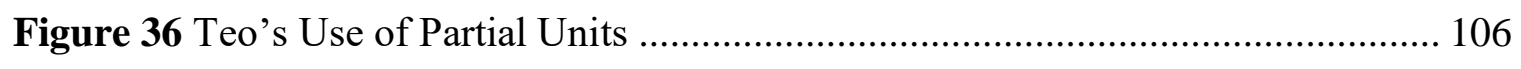

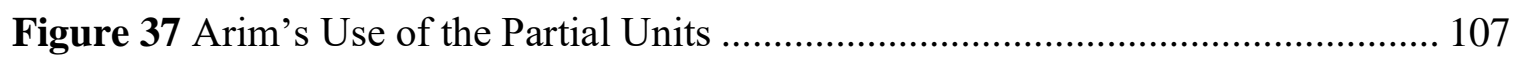

Figure 38 Gina's Use of the Given Unit ................................................................. 110

Figure 39 Alex's Use of Partial Units ....................................................................... 111

Figure 40 Emily's Change in Strategy Types, From Unit Length to Partial Units ........ 115

Figure 41 Emily's Measurement With a Whole Unit and a Partial Unit ....................... 116

Figure 42 Emily's Measurement With Other Partial Units........................................... 118

Figure 43 Emily's Measurement With a Partial Unit .................................................. 121

Figure 44 Sewoong's Unit Partitioning ………………........................................... 124 
Figure 45 Sewoong's Method of Checking the Leftover Length With an Additional

Dynamic Ruler

Figure 46 Sewoong's Subsequent Partitioning.

Figure 47 Sewoong's Comparison Between the Given Unit Size and the Equally

Partitioned Size

Figure 48 Fran's Change of Strategy Types from Determination to Estimation 131

Figure 49 Fran's Measurement With an Intermediate Unit

Figure 50 Fran's Measurement With a Given Unit Length and Partial Unit. 135

Figure 51 Alex's Change of Strategy TypesFrom a Single Unit to Multiple Units 138

Figure 52 Alex's Measurement With a Partial Unit

Figure 53 Alex's Measurement With Multiple Partial Units 141

Figure 54 Charim's Change in Strategy Types

Figure 55 Charim's Measurement With Green and Blue Dynamic Rulers 144

Figure 56 Charim's Measurement With Green and Blue Dynamic Rulers 146

Figure 57 Instrumented Mediation Process of Conceptualizing Fraction as Measure with a Digital Tool 151 


\begin{abstract}
This study examines students' mathematical strategies within a novel technological environment designed to support understanding of relationships between quantities and adjustable measuring units. In particular, 30 elementary students (grades 35) engaged in a series of fraction-as-measurement tasks to provide a cross-sectional snapshot of how they used a "dynamic virtual ruler" that could be continuously dilated. Screencast recordings were collected from a task-based clinical interview and analyzed to investigate children's mathematical actions and mathematical ideas. Students' strategy patterns were characterized using four distinct types (Not Attending, Estimating, Determining, and Commeasuring) based on their solution strategies. In addition, I attended to their change of strategy types depending on the characteristics of the tasks (within the same task series, across task series). I also articulated possible mechanisms for changing strategies across the tasks from both instrumental and semiotic approaches. My findings provide theory-driven, empirically-tested set of tasks that can be used to introduce fractions is an alternative way in elementary mathematics classrooms, and eventually drawing insight for students to use dynamic technology meaningfully.
\end{abstract}




\section{CHAPTER 1: INTRODUCTION}

Fractions are prevalent throughout everyday life (e.g., half a gallon of milk, one quarter of a dollar). When teaching mathematics in school, the Common Core State Standards for Mathematics suggests a longitudinal focus for fraction instruction from third grade to middle school. A strong foundation of fraction concepts is essential because fractions help children understand the nature of numbers, their interactions, and even advanced mathematical content areas (e.g., algebra, probability). Despite the importance of these concepts, in elementary mathematics classrooms, fractions have been viewed for decades as one of the most challenging topics for instructors to teach as well as for students to learn (Charalambous \& Pitta-Pantazi, 2007).

To illustrate, consider the following: What do you imagine when you hear the term "half?" You might come up with a circle that has a shaded half (D). Then, can you add one half and the other half (D)? Which result do you get among the possible answers of one, two halves, and two fourths? Definitely, the answer is either one or two halves. Some younger children, however, reply with the answer two fourths. Why do the children think this way? What kind of knowledge or understanding do the children draw upon when adding two fractional amounts? Even though other children may visualize fractional amounts or use mental or physical models to arrive at the correct answer, they may inappropriately link this intuitive thinking to additive operations in the rational number system.

The above example is an unsurprising story in mathematics classrooms. Researchers reported this intuitive understanding of fractions among younger students (Pitkethly \& Hunting, 1996), secondary students (Siegler \& Pyke, 2013), and even adults 
(DeWolf \& Vosniadou, 2015). In its 2008 final report, Foundations for Success, the National Mathematics Advisory Panel indicated that algebra is the gateway for later success in mathematics but also pointed out that U.S. students often struggle with algebra due to poor proficiency with fraction concepts.

What influences children's struggle to understand fractions? One possibility is the current dominant approach in textbooks of introducing fractions via a part-whole conception (Fuchs et al., 2013). Represented with static and pictorial representations, the part-whole conception describes a fraction as "a number of equal parts of a unit out of the total number of equal parts" (Lamon, 2012, p. 145). For example, the fraction $3 / 5$ would be interpreted as 3 parts out of 5 equal parts; a pair of numbers rather than a single value. Although research has shown cases where students seemed to achieve conceptual development through this interpretation (e.g., Piaget et al., 1960), other studies have provided counterexamples in which students struggled with flexibly applying the partwhole conception to other fraction domains (Brenner et al., 1999; Kieren, 1993). Because textbooks play a significant role in providing an opportunity to learn (Schmidt et al., 1997) and influencing achievement (e.g., Tarr et al., 2008), the dominant part-whole conception might limit children's understanding of a fraction as a quantity, measure, or amount (Behr et al., 1992).

Younger children especially might think of a fraction as an arrangement of two parts (e.g., its shaded parts and the equal-partitioned whole shown here: $\mathbf{O}$ ) rather than a single number or quantity. Looking back at the task of adding two halves, some students might incorrectly see two fourths as the answer. For these students, their visualized representation derived from the part-whole conception could limit their interpretations so 
that they conceive $\bigcirc$ as 2 out of 4 pieces without considering the referent unit $(\bigcirc)$. Furthermore, these students might struggle to understand that two fourths are equivalent to other fractions, such as a half or four eighths. In addition, children might have a difficult time transferring their understanding of the whole-number system to the rationalnumber system after learning counting, whole numbers, and basic arithmetic operations (Ni \& Zhou, 2005). Students' lack of opportunities to interpret the multifaceted concept of fractions might also constitute a negative influence on what students learn about advanced concepts and operations (Thompson \& Saldanha, 2003).

\section{Measurement Approach to Fractions through Linear Representations}

To develop a more robust understanding of fractions, children need more opportunities to develop mathematical proficiency with them, including an understanding of units and adaptive reasoning to judge the relative sizes of fractions (National Research Council, 2001). Children must use concept-rich activities to fully comprehend the relationships between two quantities with the goal of understanding a fraction as one quantity (Barnett-Clarke et al., 2010). It would be helpful to identify a new hypothetical learning trajectory that deviates from the traditional emphasis on the part-whole conception so that children's thinking about fractions develops into a more comprehensive understanding (Battista, 2012). In particular, there are reasons to expect that the use of linear representations and measurement tasks might support a more robust and flexible conception of fractions.

The Common Core State Standards for Mathematics emphasize the use of visual fraction models, such as a tape diagram, a number line, and an area model. However, to be effective, the explicit goal for using the models must be to represent fractional 
meanings; in other words, the students must purposefully use the models to demonstrate to themselves what a fraction means rather than just mechanically using the models (Webel et al., 2016). Providing rich experiences with various quantities (continuous and discrete) can play an important role in conceptualizing the meaning of fractions (Saxe et al., 2005). However, nonlinear continuous models (e.g., circle) have been found to promote limited understanding of fractions - for example, students have been found to develop an erroneous assumption that a fraction is always less than a whole (Pitkethly \& Hunting, 1996). There has been a call for examining an alternative approach with a linear continuous quantity (e.g., a number line and a tape diagram) rather than a nonlinear one or a discrete one (e.g., collections of individual objects). For example, Moss (2005) suggested introducing fraction concepts by using linear models based on multiplicative reasoning because other models might have limitations when used in the context of improper fractions. In the current study, I focused on a linear model to explore relationships between units and quantities, which is useful in making and identifying proper and improper fractional quantities.

A body of literature has investigated alternative approaches to developing a fundamental understanding of fractions as quantity, not merely an arrangement of whole numbers. Ni and Zhou (2005) identified two distinct alternative approaches in learning and teaching fractions: an equal sharing approach and a measurement approach. The equal sharing approach (Empson, 1999; Empson et al., 2006) focuses on the coordination of quantities (the number of items to be shared and the number of people sharing them). For example, students might engage in the following problem: If four children equally share 3 lbs of clay, how much clay does each child get? A student might solve this task 
by first partitioning $2 \mathrm{lbs}$ of clay into halves and then partitioning the last pound of clay into four parts. They would yield a half and a fourth per child as the solution. Another student might partition each pound of clay into four pieces and apportion three quarters as each child's equal share. Students can learn fractions as a different kind of quantity with this equal sharing approach, which prompts students to map the action and the result of partitioning into fractions (Empson, 1999).

In contrast, the measurement approach (Davydov \& Tsvetkovich, 1991) emphasizes a fraction as a single quantity. There are various definitions of measurement approach to fractions. Kiren (1976) was the first to conceptualize the five interrelated subconstructs of fraction concepts, which include part-whole, measurement, quotient, ratio, and operator. From this fraction as measure interpretation, students might represent fractions on a number line with a subdivision of a length unit (Lamon, 2012). However, there are several distinctions from the instructional approach by Davydov and Tsvetkovich (1991) around the idea of fraction as measure compared to Kiren's (1976) definition of fraction as measure.

First, the researchers' approach (Davydov \& Tsvetkovich, 1991) was not restricted to only fraction content but penetrated the whole-number system and rational numbers. Consider, measuring the length A (Figure 1-left) with four iterations of a single unit $(k)$. The length of $\mathrm{A}$ is 4 times the unit $k(\mathrm{~A}=4 k$ or $\mathrm{A} / k=4)$, so length $\mathrm{A}$ can be represented as the whole number 4 in terms of unit $k$. If length B (Figure 1-right) is measured with the unit $m$, there is a leftover part. This remainder could be measured with a new smaller unit $(n)$ and compared with the original unit $(m)$. If $n=1 / 2 m$, then $\mathrm{B}=m+m+n=2 m+n=2 m+1 / 2 m=(2+1 / 2) m=2 \frac{1}{2} m$. In other words, the length of $\mathrm{B}$ 
can be represented as the fraction $2 \frac{1}{2}$ in terms of the unit $m$. Both whole numbers (in the case of length A) and fractions (in the case of length B) have the same approach of generating numbers using measurement.

\section{Figure 1}

Measuring Length A With a Single Unit (Left) and B With Two Units (Right)
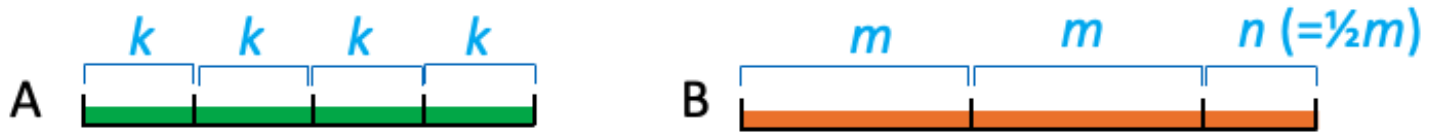

Second, using the approach defined by Davydov and Tsvetkovich (1991), students can experience cognitive dissonance between whole numbers and fractions by exploring the relationships between units and quantities that cannot be measured using whole iterations of the unit. As in the example of length $\mathrm{B}\left(=2 \frac{1}{2} \mathrm{~m}\right)$ in Figure 1 , the initial fraction concept is introduced by defining the relationship between the original unit and a new unit.

In this study, I drew upon the measurement approach to fractions (Davydov \& Tsvetkovich, 1991), which focuses on the relationship between the units of measure and the magnitude of quantities. This approach not only emphasizes children's experiences and the meaning of fractions but also mirrors the historical development of fractions as a consequence of measuring continuous quantities. As a long-term goal, the overarching question I had pursued was how we could provide meaningful learning opportunities for students to explore this quantitative relationship using a measurement approach. Therefore, in this study, I created a technological tool embedded the features of 
measurement approach, then designed a series of measurement tasks, and examined how children were engaged fractional understandings with the tool.

\section{The Use of Dynamic Technology to Enhance Mathematical Understanding}

Due to the accelerating pace of social and technological change, students need to develop a deeper understanding through critical thinking, not just rote memorization. The incorporation of technological tools into classroom learning has been investigated as a way to help students visualize mathematical relationships and to provide a foreground for displaying, acting upon, and observing the relationships (Heid \& Blume, 2008). These tools allow us to explore a new way to conceive math concepts that we could not explore without them (Hollerbrands, 2007; Webel \& Otten, 2015). The National Council of Teachers of Mathematics (NCTM, 2014) emphasized tools and technology as one of the essential elements to support effective learning for all students. It also has been recommended that technology helps students make sense of mathematical ideas and reason mathematically.

In a traditional pen-and-paper environment, students might generate a single fraction value at a time with a static representation (e.g., fraction strips). However, in a dynamic technology environment, students can generate multiple and continuous values merely by dragging a single point. During such manipulation, students could discover a certain property across the generated examples and could develop mathematical concepts through this mediation of tools (Roschelle et al., 2017). This dynamic technology environment can change the way students learn, including discourse and learning strategies (Jahnke, 2015). 
To examine how technology enhances mathematical learning, the instrumental perspective and the semiotic perspective provide useful theoretical lenses. More specifically, the instrumental genesis considers how a tool changes from an artifact to an instrument in the hands of a student. Semiotic mediation refers to how a tool mediates the child's mathematical understanding. They share the same starting point: the Vygotskian perspective, which posits that artifacts play an essential role in developing knowledge (Vygotsky, 1978). Each framework provides a distinctive construct to delineate a wholistic picture relevant to the instructional use of artifacts.

Many researchers have discussed the importance of fraction learning using different approaches. Many have suggested that dynamic tools are promising in forming students' conceptual understanding of fractions as well as their procedural knowledge. These researchers' findings raise a question: How might technological tools support students' learning about fractions through a measurement approach? Relatively little research has examined elementary students' understanding of fractions from a measurement approach, and much less research has investigated elementary students' understanding of fraction as measure within a technological environment (Simon et al., 2018).

Therefore, the current study examined how elementary students coordinate units and quantities in situations that measure length when solving a series of fraction tasks. The study used a dynamic tool as a new pathway for understanding fraction concepts. In terms of theoretical contributions, this study (a) initiates new discourse about how children construct fraction concepts in measurement situations, (b) identifies the potential use of dynamic technology in discovering mathematical properties with continuous 
variation, and (c) develops a holistic framework for mathematical concepts and the use of dynamic technology for further study and analysis. From a practical perspective, this study may help practitioners to understand mathematical learning in a dynamic technology environment. Practitioners (e.g., teachers, researchers, and curriculum developers) could be ready for implementing this measurement approach to fractions via technology in their fields with basis on the understandings of the nature of students' conceptualization of fraction as measure. Beyond instructional potential, this study can build a bridge between technology and educational access and equity by providing highquality learning opportunities for all students (Schoenfeld, 2002).

\section{Research Questions}

This study employed a series of tasks focused on eliciting and supporting fraction understanding through measurement situations (Davydov \& Tsvetkovich, 1991). I

developed tasks that employed Dynamic Rulers (Yeo, 2020)—a set of measurement tasks that I created for this dissertation with the New Cabri software (Laborde, 2016) — which allowed study subjects to explore mathematical relationships and properties with dynamic actions. Like a ruler, this tool was designed to measure unspecified lengths with adjustable units; adjustments to one unit of a Dynamic Ruler simultaneously changed the unit size throughout the whole ruler. From the instrumental and semiotic perspectives, I examined how students coordinated and described the relationship between quantities while using the Dynamic Rulers. In particular, I addressed the following questions:

1. What are the different ways that children use the Dynamic Ruler tool in approaching each fraction-as-measurement task? 
2. What patterns emerge in children's strategies across the series of fraction-asmeasurement tasks?

\section{Researcher Positionality}

Since 2006, as a former elementary school teacher in South Korea, I have conducted a variety of research projects about how to use cognitive and social technology for better learning and teaching in mathematics classrooms. During my doctoral program, my positionality has shifted from that of a practitioner to a novice researcher who pursues research to focus on not only practical solutions but also theoretical ideas in order to contribute to both communities. Through these teaching and learning experiences, I have honed my abilities to develop my own tool for a specific purpose, to understand mathematical discourses emerged by the use of a tool with a wide range of individual students, and to analyze collected data with a rich and thick description from the viewpoint of both an insider and outsider.

I came to the US to study mathematics education as an international student and observed plenty of elementary classrooms as a newcomer. These experiences forced me to reflect on what was taken for granted related to cultural and linguistic factors in learning. In this study, I ended up with recruiting a diverse set of participants from diverse ethnicities, however, I was not drawing conclusions from a population that only mirrored my own beliefs and background. Conversely, this diverse recruitment strategy might allow me to create new paths to understand complicated and abstract concepts in the fraction content area by investigating a variety of students' strategies and mathematical actions, which brought to the surface their strategies with a tool more universally regardless of their ethnic backgrounds. Furthermore, I also could draw a 
contextualized insight to support those students who have different cultural and social backgrounds. 


\section{CHAPTER 2: LITERATURE REVIEW}

The purpose of this study was to investigate how the children's concept of fraction as measure emerged when working with dynamic technology. This study also focused on how the tool mediates such mathematical conceptions. In this chapter, I first describe an integrated framework for conceptualizing mathematical understanding that arises from the use of technology. I used two theories for the framework: instrumental genesis and semiotic mediation. I then elaborate on the challenge of a typical approach to fraction learning and teaching in schools. Afterward, I provide a synthesis of the theoretical and empirical literature concerning how the concept of fractions develops through a measurement approach. Then I elucidate the dynamic features of technology and review the use of digital tools to develop fraction understandings.

The conceptual framework first described in this chapter relates to the integration of the study's technological tool with an understanding of mathematical concepts from both instrumental and semiotic perspectives. The synthesized literature is describing how students can build up mathematical concepts by using a dynamic technology environment.

\section{Conceptual Framework}

This study's conceptual framework relates to the interaction between students and technological tools for developing mathematical understanding, largely inspired by Drijvers et al. (2010). They provided a historical overview of theoretical frameworks regarding the integration of technology in learning and teaching mathematics, which has contributed to a new perspective in the mathematics education field (e.g., the perspective of a microworld within the digital environment where mathematical concepts can be 
explored). Drijvers et al. (2010) specifically focused on the theory of an instrumental approach and the theory of semiotic mediation "to guide the design of teaching, to understand learning, and to improve mathematics education" (p. 90). Both theories have the same starting point: the Vygotskian learning theory, which leverages the impact of tools. The instrumental perspective (Verillon \& Rabardel, 1995) emphasizes the transformation of an artifact to an instrument in relation to students' action schemes and the tool's affordances and constraints. The semiotic perspective (Bartolini Bussi \& Mariotti, 2008) focuses on how a psychological tool mediates children's mathematical meaning making. Therefore, building an understanding of mathematical concepts with tools can be thought of as analogous to considering the iterative interactions between students and tools (Figure 2).

For this study, I integrated the two theories into the conceptual framework because they had a complementary relationship with each other (Maschietto, 2015). First, the instrumental genesis theory took into account students' cognitive processes when they are involved in tool-based mathematical activities. Second, the semiotic mediation theory supported how tool-based mathematical tasks can foster the emergence of mathematical meanings through the use of signs. 


\section{Figure 2}

\section{Conceptual Framework}

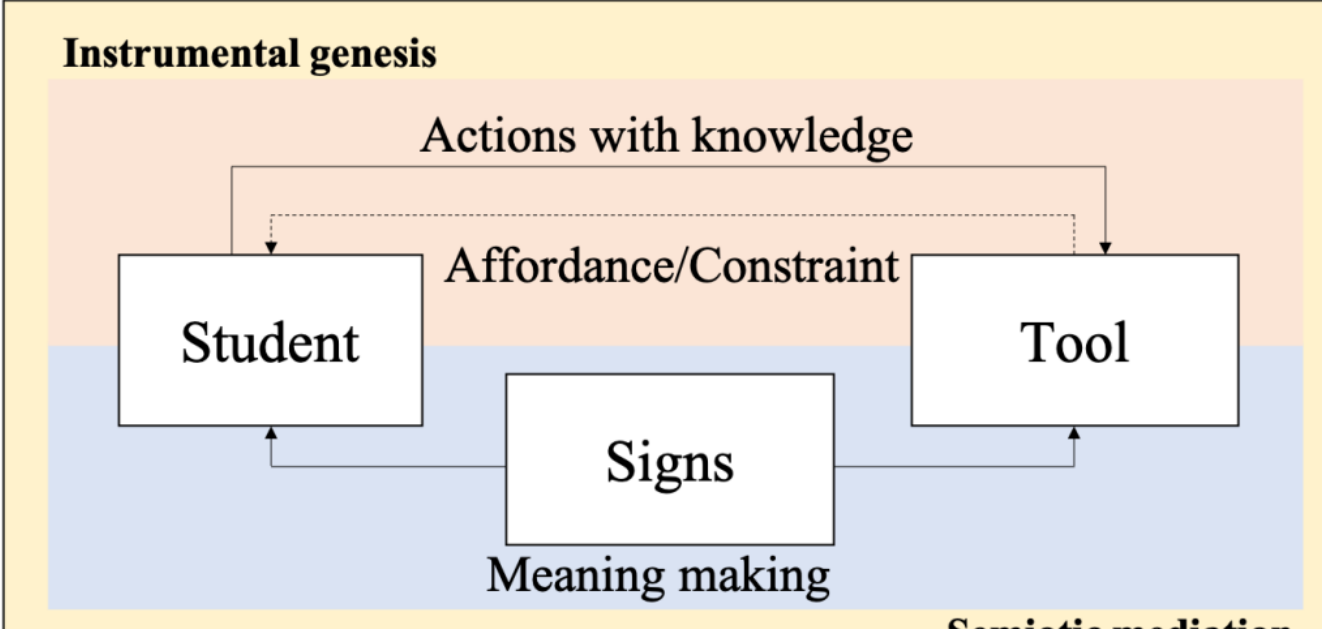

\section{The Importance of Tools}

Vygotsky $(1978,1981,1987,1997)$ elaborated a theory about how children learn, how they develop their minds, and the interconnection. He said that children's minds develop through social experience with special psychological tools (e.g., language, symbols, and signs) rather than through individual growth. Such tools are provided by more knowledgeable people through joint activities. The children use the tools at the external type and then internalize the lessons learned. Vygotsky (1978) illustrated this development with the example of pointing a finger. A child might initially perceive the pointing motion as meaningless. However, if other friends and adults respond to the gesture, it becomes a sign that has meaning, such as going to the pointed direction. In particular, the pointing gesture represents an interpersonal connection between individuals with shared meaning. Vygotsky (1997) elaborated upon how children make meaning, explaining that meaning making is the unification of thinking and speech: 
Meaning is not the sum of all the psychological operations which stand behind the word. Meaning is something more specific - it is the internal structure of the sign operation. It is what is lying between the thought and the word. Meaning is not equal to the word, nor equal to the thought. This disparity is revealed by the fact that their lines of development do not coincide. (p. 133)

Therefore, the acquisition of psychological tools is a critical factor that impacts the development of children's minds and meaning making. Teachers can support this acquisition process by organizing appropriate learning activities for children to acquire and effectively use psychological tools, such as language and symbols.

Building on the Vygotskian foundations, in the following sections, I elucidate two main theories: instrumental genesis and semiotic mediation. Then I integrate the two theories to form the conceptual framework for this study.

\section{Instrumental Genesis}

The theory of instrumental genesis is the first part of the framework. It refers to the process of an artifact transforming into an instrument (Artigue, 2002; Drijvers \& Gravemeijer, 2005; Drijvers \& Trouche, 2008; Trouche, 2004). For example, if an artifact influences a student's mental processes and impacts the student's actions (e.g., mathematical activity), then the artifact can be connected to the students' cognitive processes and becomes an instrument (artifact + scheme). Here, it is important to distinguish between the artifact and the instrument (Verillon \& Rabardel, 1995). The artifact is a physical or psychological object that has an apparent and practical role for achieving a given task, whereas an instrument is transformed from the artifact when it is 
used purposefully. Drijvers et al. (2013) exemplified the difference between an artifact and an instrument:

As long as we have no idea about what letters stand for, a pen is a useless artefact for writing. As soon as we learn to write, the pen becomes more than an artefact for drawing, and changes into an artefact that we also use for writing. Together with the developing skills, the pen becomes an instrument for writing (p. 26). Then what is the process of becoming an instrument from an artifact? Consider a child who is measuring the length of a pen with a ruler that features inches on one side and centimeters on the other. The child measures the pen and finds out that the length is 3 in. $(7.62 \mathrm{~cm})$. This finding guides the child's thinking toward seeing the measurement of the pen as three iterations of inch units. Later, the child measures the same pen with the centimeter side of the ruler, encountering an opportunity to realize the unit relation between inches and centimeters. Furthermore, the child decided to mark millimeters at the side of centimeters to measure the length of the pen more precisely, then come to realization of the relationship between inches, centimeters, and millimeters. During instrumental genesis, the artifact (e.g., a ruler) and the child establish a bidirectional relationship: instrumentation and instrumentalization. Instrumentation is when the child's understanding of mathematical concepts is shaped by the tool's affordances and constraints. In the above example, this could be seen when the child first measured the pen in inches to quantify the length with the ruler only. Then, the student had an opportunity to perceive a relationship between the size of the initial unit, the measure, and alternative units by comparing the measurement in centimeters as well as millimeters. That is, the child's knowledge guided how the tool is used and shaped the 
tool for the purpose, which is known as instrumentalization. During this process of instrumental genesis, the child's thinking can be shaped by the artifact and shape the artifact as well (Hoyles \& Noss, 2003).

In addition to an individual type of the instrumental approach, students construct their own instrumental genesis then collaborate with their classmates in a classroom. Trouche (2004) coined the term instrumental orchestration, which means that teachers intentionally and systematically organize various artifacts to develop students' instrumental geneses. Therefore, in digital technology learning environments, it is required for teachers to design instrumental orchestration to use a given tool meaningfully.

When students are given an artifact to solve a mathematical task, the process of internalizing (instrumentation) and externalizing (instrumentalization) is reflexive between the student and the artifact. For example, Freiman et al. (2017) investigated how computer-based story problems help students analyze mathematical structures and relationships with algebraic thinking rather than with calculations. They believed younger children could solve a story problem with generalized strategies using letters in the expressions. Within the computer environment, students solved a task: "Sarah had $s$ apples. She ate $f$ apples in $d$ minutes. How many apples does she have now?" The task's goal was to make a mathematical expression by dragging the letters $(s, f, d)$ and operational symbols $(+,-, \times, \div)$ into the correct equation. The solution of the task was $s-f$. This computer-based environment situated students so that they could construct an algebraic expression with variables without any calculation (instrumentation). Even though students had an option to replace the letters with numerical values, some of them 
were able to keep focusing on the analysis of the problem and the general additive structure with the algebraic expressions. The computer environment could shift students' attention from numerical computations to figuring out algebraic relationships. As the process of instrumentalization, before moving to the algebraic expression on the computer, students drew a number line model to understand the underlying relationships between a minuend, a subtrahend, and a difference in the problem situation. This was helpful to understand mathematical structure of the given problem and eventually influenced the way to solve the computer tasks by using letters.

In another example, Soury-Lavergne and Maschietto (2015) studied the use of a digital graphical space as a bridge between the physical space and the geometrical space. They examined the use of the digital space designed by the Cabri Elem software (graphical space) to connect a green-carpet activity (physical space) in the classroom to the concepts of lines and intersections (geometrical space), focusing on grids. In the actual classroom, students solved a spatial problem by tracking the position or path of an object using a grid on a green carpet. Subsequently, the students solved digital tasks grouped under the title "Cabri and the Frog," manipulating a frog through the digital grid to reach Cabri (cabri means "goat" in French). Through these tasks, the students recognized the grid as an object that belongs to the digital graphical space and explored potential obstacles (e.g., gray squares and clouds) and affordances (e.g., changing the point of view from $2 \mathrm{D}$ to $3 \mathrm{D}$ and vice versa) via instrumentation. The students could utilize their strategies gleaned from the classroom green-carpet activities via instrumentalization. For example, some students used a reference-points strategy to navigate Cabri using black lines or gray squares at the corners. In this example, the use of 
digital technology supported instrumental genesis by constructing connections between the physical carpet activity and the geometrical concept of a grid.

In summary, prior studies related to the instrumental approach highlighted the process of instrumentalization and instrumentation. The studies evidenced ways to utilize technology according to one's own purpose and understanding of mathematics and identified affordances and limitations to influence their understanding. In this study, I also focused on how children's strategies about fraction concepts influenced the manipulation of a dynamic tool. This study further examined which of the tool's affordances and limitations impacted the children's understanding of fractions.

\section{Semiotic Mediation}

In the second part of the framework, semiotic mediation refers to the process of meaning making from signs. Vygotsky (1978) differentiated between tools and signs as follows: "The sign acts as an instrument of psychological activity in a manner analogous to the role of a tool in labor" (p. 52). Signs are symbolic tools (e.g., speech, number) to be used in mental work while tools are practical, material tools (e.g., computer, ruler) to be used in physical work. These symbolic and material tools can mediate children's understanding. Let us suppose that a child is solving an addition problem. The child might manipulate a snap cube (material tool) or use a number expression with mathematical signs (symbolic tool).

When it comes to the use of technology in particular, the signs generated by manipulating technological tools are internalized and directed to other signs (e.g., words, drawings, and gestures) in the social activity. Bartolini Bussi and Mariotti (2008) described semiotic mediation as a process of meaning making through internalizing the 
signs that are produced from external, interpersonal activities. In the case of drawings with Dynamic Geometry Software (DGS), a goal-oriented activity such as dragging and tracing in DGS can be internalized to construct individual meanings. The use of dragging and tracing tools also facilitates whole-class discussions to mediate the collaborative meaning-making process in the activities.

Extending the Vygotskian perception that signs are symbolic tools, Bartolini Bussi and Mariotti (2008) suggested three categories of signs: artifact, mathematical, and pivot signs. Artifact signs are generated in the context of using an artifact and are close to the artifact itself. Mathematical signs are generated in abstract mathematical contexts. Pivot signs "express a first detachment from the artefact, but still [maintain] the link to it in order not to lose the meaning" (Bartolini Bussi \& Mariotti, 2008, p. 757). In other words, pivot signs form a bridge between artifact signs and mathematical signs. In the classroom community, certain words and tools can mediate the meaning-making process. Arzarello and Robutti (2008) termed this collection of signs and their reciprocal relationships as the semiotic bundle. Therefore, the goal of teaching can be to transform tool-generated signs to mathematical signs through learners' meaning-making processes.

Semiotic mediation through technological tools is illustrated by a teaching experiment by Bartolini Bussi and Baccaglini-Frank (2014), who investigated the use of a programming robot (the bee-bot) to transition children's perceptions of paths from a dynamic perception to a geometric perception (i.e., seeing paths as geometric figures such as squares and rectangles). They analyzed three different signs from the programming activities. On a paper, children wrote down arrows $(\curvearrowleft, \uparrow, \downarrow, \curvearrowright)$, which stemmed from the command-icons on the bee-bot's back (i.e., artifact signs). The arrows 
that the children wrote on paper represented paths as sequences of commands (e.g., a square: $\uparrow \uparrow \curvearrowright \uparrow \uparrow \curvearrowright \uparrow \uparrow \curvearrowright \uparrow \uparrow \curvearrowright$ ). Here, corresponding mathematical signs (e.g., a right angle of a square), the turning arrow $(\curvearrowright)$ become the pivot sign that derived from the artifact signs (e.g., the command-icons on the bee-bot, the action of turning right in a path on the floor). Children could enhance their perceptions of a path with the periodic eye-blinking and beep sounds of the bee-bot. Because the bee-bot produced the blinking and beeps at starting and ending points, those visual and audible patterns prompted children to reflect on the action of the bee-bot related to geometrical objects.

Digital tools can generate dynamic mediators that invoke mathematical relationships and properties to support students' understanding. Ng and Sinclair (2015) investigated how children's geometric conceptions of symmetry emerged and transformed through language, gestures, and the use of technology. They studied the semiotic role that dynamic digital technology has in learning symmetry. Children manipulated squares and/or a symmetry line in an activity titled "The Symmetry Machine.” The children dragged squares and observed what happened to corresponding symmetry points, which were positioned on the other side of the line of symmetry on an interactive whiteboard. The actions of moving the squares served as the pivot signs and produced both artifact signs (the movement of a particular square) and mathematical signs (the square as a mathematical object with the line of symmetry). The following statement from one of the participants in Ng and Sinclair's study (2015) provided an example of how their dynamic digital technology enhanced the participant's concept of symmetry: "It will move like opposite, like this one will move to the windows, and this one will move to the wall” (p. 432). 
In the process of semiotic mediation, visual representations, gestures, spoken words, and written languages are obviously crucial mediators to facilitate discussions among the whole classroom (Battista, 2008; Kaur, 2015). More importantly, the use of technology can activate new types of potential mediators. The dragging tool used in $\mathrm{Ng}$ and Sinclair's study (2015) mediated children's comprehension using the dynamic action of the line of symmetry and the image squares. This tool allowed the children to attain an understanding of the main features of symmetry. The shape of a component on one side of the line of symmetry is the same as its counterpart on the other side of the line; a component is the same distance away as its counterpart; the preimage and image make a pair.

Findings from these studies highlighted the important role of words and mediators in semiotic activities when solving tasks in technological environments. In this study, I investigated how children transformed and evolved visual representations into mathematical words to make a mathematical meaning by manipulating a dynamic tool in a digital environment.

\section{Instrumented Mediation}

The integrated framework that connects instrumental genesis to semiotic mediation provides a useful way of understanding of children's use of digital tools. In this study, I called this integrated approach instrumented mediation. When solving technology-embedded tasks, the students who participated in my study needed to solve the tasks based on their mathematical knowledge and to manipulate a digital tool within a digital microworld. As a result of the manipulation, the students generated new signs 
such as words, symbols, and visual representations. The new signs allowed them to make mathematical meaning through instrumented actions.

Instrumental genesis has a strong orientation toward the cognitive process of how the use of instruments mediates mathematical learning and teaching. In contrast, semiotic mediation is a theoretical approach focused on how the process of meaning making occurs through the internalization of different types of signs. However, some researchers have investigated ways to use both theories as one framework. For example, Drijvers et al. (2013) used the two theories to analyze one episode of students' learning with a computer algebra system. By comparing the two theories' principles and methods, they reflected on the potential benefits and pitfalls of applying the theories to analyze the learning episode. For example, from the instrumental perspective, manipulation was presented as the observable part of a student's mental scheme, which the student used to solve a given type of task. On the other hand, from the semiotic perspective, the meaningmaking process was understood as a sequence of actions governed by rules and oriented toward a goal. Even though the notion of manipulating tools was focused on the use of tools, the notion of meaning making was broader. Therefore, Drijvers et al. (2013) concluded that the notions of manipulation and meaning making could provide a comprehensive view of how students use tools to develop understanding.

Similarly drawing on the relationships between the two theories, Maschietto and Soury-Lavergne (2013) used teaching experiments to examine the use of a physical manipulative (the Pascaline) and a digital counterpart (the e-Pascaline). Manipulating a gear train of five wheels, both tools allow numbers to be written in the decimal position system and arithmetical operations to be performed. When analyzing the use of the tools, 
the authors identified how a participant could use a forefinger to turn wheels (one-toothat-a-time gesture). This manipulation could support some mathematical meanings (e.g., place value and operations), and the tools also provided a chance to make meaning from linguistic expressions (e.g., turn $n$ times), which were related to iteration. This evidence suggested that tools could help the researchers understood how participants were constructing mathematical meaning through instrumental genesis (i.e., asking a participant to manipulate the machine) and semiotic mediation (i.e., prompting the participant to argue why they chose the manipulation that the did).

Following prior studies' efforts to integrate the two theories, this study also focused on how children derive mathematical meaning from the relationship between mathematical ideas arising during situated tasks and mathematical actions performed within a dynamic digital environment. In other words, this study focused on instrumented mediation which integrated theories of instrumental genesis and semiotic mediation.

In the next sections, I discuss research on the development of fraction concepts from measurement approach and the features of dynamic technology. Then, I reviewed relevant studies to develop fractions concepts with digital technology.

\section{A Measurement Approach to Fractions}

\section{Challenges and Alternative Approaches for Learning Fractions}

Many mathematics education researchers have documented that learning fractions are one of the most complicated areas for younger students (Charalambous \& PittaPantazi, 2007). When fractions are initially introduced, students sometimes struggled with applying concepts and properties transferred from the whole-number system and had a limited understanding of fractions as a relationship between two numbers rather than as 
a single quantity (Behr et al., 1992; Clarke \& Roche, 2009; Ni \& Zhou, 2005; Tzur, 1999). The difficulty in learning fractions arises from whole-number bias (Ni \& Zhou, 2005) and confusion about the referent unit (Tzur, 1999). For example, due to their lack of understanding that a fraction is a quantity, children may see the numerator and denominator as an arrangement between two separate numbers, not as a single quantity, amount, or measure. Consequently, they may only pay attention to the number of objects, not the size -1 out of 4 objects rather than one part that is one fourth of the size of the whole.

Instruction can exacerbate such inherent difficulty by employing the doublecounts treatment in the classroom (i.e., the denominator is counting how many parts in the total and the numerator is counting how many parts are shaded; Kieren, 1994). This type of instruction focuses only on the part-whole meaning of fractions (Lamon, 2007; Mack, 2001; Olive \& Vomvoridi, 2006). The double-counts treatment seems to have intuitive effects for students, from which they can build fraction understanding of an existing counting scheme and can introduce fractional words (e.g., "three over five", "three out of five". or "three fifths"). Chval et al. (2013) illustrated how the double counts approach causes confusion about discrete quantities. Their Fraction Hexagonal Task (p. 50, Figure 3) illustrated the different ways that students understand fractions by perceiving a hexagon and its subcomponents as an area model. For example, one student named Diana answered the question and agreed with Mary's statement that the green triangle represented one third of the pieces: "Yes, there are 3 pieces and the triangle takes up 1 part." However, Diana disagreed with Michelle's statement: "No, there are not 6 pieces total, she said". The student seemed to think of the model as discrete that the 
triangle is $1 / 3$ of the pieces rather $1 / 3$ of the area and to interpret the denominator as the number of pieces rather than as an equal-partitioned area. The lack of opportunities for students to interpret fractions in other ways (measure, ratio, operator, and quotient) might be a negative influence on what students learn about advanced concepts and operations (Ni, 2001; Thompson \& Saldanha, 2003).

\section{Figure 3}

\section{Fraction Hexagonal Task}

Q. Read what Mary and Michelle say about the green triangle.

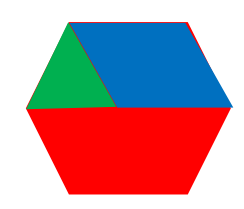

Mary: The green triangle represents $1 / 3$ of the pieces.

Michelle: The green triangle represents $1 / 6$ of the hexagon.

(a) Do you think Mary is correct? Explain your thinking.

(b) Do you think Michelle is correct? Explain your thinking.

Researchers have explored alternative instructional interventions to develop a fundamental and conceptual understanding of fractions. Two distinguished approaches have emerged. One is the equal sharing approach (Empson, 1999; Empson et al., 2006; Streefland, 1991, 1993), which emphasizes the coordination of quantities in equal sharing problems (e.g., 4 children share 5 pancakes so that everyone gets that same amount. How much pancakes can each child have?). The other is the measurement approach (Davydov \& Tsvetkovich, 1991; Morris, 2000; Simon et al., 2018), which emphasizes mathematical generalities across whole numbers and rational numbers. More specifically, the fundamental idea for the equal sharing approach is partitioning with multiplication and 
division. Empson and Levi (2011) suggested that students require the opportunity to understand the meaning of fractions prior to introducing fractional notation. Focusing on the development of children's mathematical thinking, they posed equal sharing problems and studied how students constructed fraction concepts by "slicing, splitting, distributing, measuring, and combining quantities" (Empson \& Levi, 2011, p. xxii). Equal sharing tasks generate the fractional amount as shares and allow students to reflect on the relationship between the denominator (the number of pieces in a whole object) and the numerator (the number of pieces each sharer gets).

On the other hand, the measurement approach is based on the concepts of measurement units and unit hierarchies, which draws not only on children's experience and the meaning of fractions but also on the historical development of fractions through measurement (Davydov \& Tsvetkovich, 1991). This interpretation emphasizes the relationship between the units of measure and the magnitude of quantities. To illustrate, suppose a student measured length A with unit $k$ (Figure 4). After two iterations of unit $k$, the student found a smaller unit was necessary to measure the remainder, and this new unit was a third of the size of the original unit. This approach might be helpful for children to understand fractions with an emphasis on the concept of measurement units and the relationships between them. 


\section{Figure 4}

Measuring A With Unit $\mathrm{k}$ and a Partial Unit

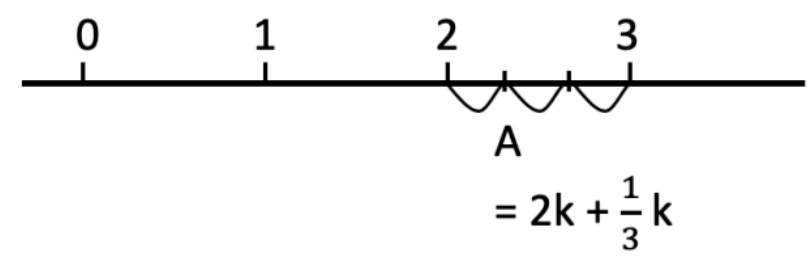

\section{Theoretical Learning From a Measurement Approach}

Some researchers have argued for considering measurement as the source of number concepts, including all number systems in general and fractions in particular (Davydov \& Tsvetkovich, 1991; Brousseau et al., 2014). Historically, fractions were invented to measure quantities or amounts, such as how many grains or how much liquid, which have been measured since ancient Egypt (Ifrah et al., 2000). In school mathematics, the units of measure and the magnitude of quantities remain fundamental constructs to understand the relationships between numbers and operations (Kaput, 1985; Schwartz, 1988). However, this idea of measurement as the source of number concepts has been supplanted in school by the part-whole interpretation due to the development of modern algebra from integers to rational numbers. In addition, as the field of modern algebra grew in prominence, rational numbers became more formalized by holding their consistent properties as a new number system across integers and real numbers. As a result, educators came to view measurement as unnecessary to contribute to the formation of rational number concepts, which obfuscated their practical origins.

Davydov and Tsvetkovich (1991) focused on promoting students' understanding of mathematical structure rather than extending number systems in a prescribed order (e.g., whole number, then rational number, etc.). That is, if students learn a generalized 
and abstract structure of a mathematical number system from experiences that focus on essential and conceptual understanding, they might apply this structure across all number systems. For example, students might approach the following operations differently using conventional number systems: $1+2,1 / 3+2 / 3,0.1+0.2, x+2 x$. However, these operations have the same structure across different number systems: one unit + two units.

The idea to approach problems in a general way is supported by Davydov's (1988) two basic ways to develop students' learning: empirical learning and theoretical learning. Empirical learning includes comparing various objects, figuring out commonality, and formulating a general concept (Karpov \& Bransford, 1995). As a constraint, this empirical approach might result in erroneous concepts. For example, preschoolers might tend to believe that "all small objects sink," and younger elementaryschool students might believe that "the whale is a fish" (Karpov \& Bransford, 1995, p. 62). On the other hand, theoretical learning provides psychological tools, which are general and essential methods for solving concrete problems and would be internalized by students in the process of the application. For example, kids could investigate the importance of density to understand why the size of objects does not matter when considering its propensity to sink. They also could think about the criteria for classifying mammals and fishes: respiration, circulation, reproduction, etc. Students need to not only acquire scientific knowledge as they explore their world but also master the processes underlying the concepts they have begun to formulate. It is essential for students to be able to apply the concepts to concrete problems.

Drawing on this theoretical learning perspective, Davydov and Tsvetkovich (1991) implemented a teaching experiment based on the measurement of quantities as the 
origin of the fractional number system. Children first explored direct comparison in the context of length, width, height, or weight. They compared two quantities by recording the results with mathematical symbols such as,$+>,=,<$. Then, the children were introduced by new situations that made it difficult for the children to use direct comparisons. So, the children were encouraged to measure the quantity with concrete objects (e.g., stick, pieces of string). The relationship between the quantity and unit was represented with whole numbers. Next, the children encountered situations where they could not measure with whole numbers alone but had to measure a remainder with a new partial unit. If a smaller unit fitted a quantity to be measured $n$ times and the original unit $m$ times, the ratio between $m$ and $n$ could be noted as $n / m$. This measurement approach provided an abstraction of the relationship between quantities to introduce fractions as theoretical learning. The children could learn to appreciate generalized ideas across the whole- and rational-number systems, such as measure and unit of measure.

Drawing upon the measurement approach (Davydov, 1975; Minskaya, 1975), researchers developed a curriculum called Measure Up and explored elementary mathematics in grades 1-5 (Dougherty et al., 2004). A body of studies investigated the use and impact of the innovative curriculum for a variety of topics, including early algebra (Dougherty, 2008), unit concept development (Dougherty \& Venenciano, 2007), fraction multiplication (I et al., 2015), and proportional reasoning (I et al., 2018). The studies introduced children to the concept of numbers in the context of measurement by using continuous and nonspecified quantities (e.g., water, paper strips). Children explored the combination of physical, diagrammatic, and symbolic representations in measuring activities, which provided them with an opportunity to understand the structure and 
properties of mathematics. For example, Dougherty and Venenciano (2007) designed a series of comparison tasks (e.g., length, mass, volume, area) suggesting three steps for representing quantitative relationships through algebraic expressions: (1) comparing various quantities by direct measuring (e.g., $\mathrm{A}>\mathrm{B}, \mathrm{C}=\mathrm{D}$ ); (2) assessing the extent, quality, and value (e.g., A > B, B > C, so A > C); and (3) quantifying continuous quantities with a certain standard (e.g., $\mathrm{A}+\mathrm{A}+\mathrm{A}+\mathrm{A}=\mathrm{B}, 4 \mathrm{~A}=\mathrm{B}, 3 \mathrm{~A}=\mathrm{B}-\mathrm{A}$ ). From the symbolic expressions, children recognized the relationship between units (e.g., A/B = 4, and $\mathrm{A} / \mathrm{C}=6$, so $\mathrm{B}$ is bigger than $\mathrm{C}$ ) and even between unit and measure (e.g., the bigger the unit, the fewer units are needed to measure).

Extending this body of studies, other researchers have incorporated a measurement approach for fraction learning in teaching experiments (e.g., Simon et al., 2018) and classroom settings (e.g., Schmittau, 2005; Schmittau \& Morris, 2004). For example, Simon et al. (2018) designed a curriculum for teaching fractions based on a measurement approach and conducted a one-on-one teaching experiment with fourth graders. One task they posed was to measure a beam with a given length unit. Then a prepartitioned unit was used to measure the leftover in relation to the given unit. For example, a child copied a unit A (one of the gray units) to measure the given beam and figured out that the length was seven units of A. Then, the child measured another unit, called unit B (the yellow bar), determining it equaled 4 times unit A. With this information, the child could figure out the length of the beam with various units: seven units of $A$, or 1 unit of B plus 3 units of $A$, or $13 / 4$ units of B.

Students in the Simon et al. study (2018) developed an initial concept of fraction as a measure, and they were invited to further consider the relationship between 
measurement and iteration. For example, Kylie was able to conceive of a unit fraction as a partial unit that measured a whole unit (fraction as measure), "It's one third. ... Because, I'm measuring that - three of these measures one unit." She also conceived that the unit fraction was a part that iterates to a unit a particular number of times.

Findings from such studies highlight the promise of measurement approaches to fraction concepts. Students who have developed a concept of fraction as measure can think about fractions quantitatively rather than numerically (Thompson \& Saldanha, 2003).

\section{Key Constructs of Fraction as Measure}

From the measurement approach, the key constructs are related to how children think about units, measures, and the relationships between units and/or measures. The measures could be represented as whole numbers or rational numbers. However, in the case of a rational number, it would be necessary to use a smaller unit to precisely express a given quantity. When relating the new unit to the original unit, it would be an entry point to introduce the concept of fraction as a way of describing this relationship. In this section, I briefly describe which measurement concepts are crucial to extend children's understanding to fractions: length measurement, unit coordination, and multiplicative reasoning. I review the literature concerning these concepts in this section. Also, I used these concepts as the foundation for this study's preliminary data analysis framework, which is described in Chapter 3.

Length measurement consists of three parts: identifying a unit length, partitioning the object by that unit, and placing that unit iteration alongside the object (Clements \& Stephan, 2004). Unitizing is the mental process of assigning a unit to measure a given 
quantity (Lamon, 1996). In a length measurement situation, students must determine the size of one unit so that they can coordinate the unit to quantities. Partitioning means mentally or physically slicing an object into same-sized units. Unit iteration is the ability to think of the unit length as part of the length of the object to be measured and to place the unit length repeatedly along with the object to obtain the object's length (Kamii \& Clark, 1995).

Unit coordination refers to students' ability to build units and maintain their relationships with other units that they contain (Steffe, 1992). In order to conceptualize fractions, students must perceive the appropriate unit hierarchy (Hackenberg \& Tillema, 2009). At more sophisticated reasoning levels, students must operate with a number as a composite unit, which refers to a unit of units because the units might have multiple layers in the hierarchy.

Thompson and Saldanha (2003) emphasized a multiplicative structure in fraction concepts, which refers to quantifying an object with identical copies of some quantity (Vergnaud, 1994). In more sophisticated reasoning levels, students must understand a fraction as scaling $(m / n=m$ of $1 / n$ unit) rather than additive reasoning $(m / n=1 / n+1 / n$ $+1 / n+\ldots+1 / n)$. The measurement approach to fraction also provide the opportunity to consider the denominator as a unit and the numerator as a result of measuring. Therefore, students could develop multiplicative reasoning in solving the measurement tasks.

\section{Dynamic Mathematics and Principles of Dynamism}

Digital technology has been increasingly integrated into mathematics classrooms. This integration has piqued researchers' interests in understanding the role of technology in teaching, learning, and doing mathematics. Studies have given plenty of evidence that 
digital technology supports students' abilities to visualize mathematical ideas (e.g., Moreno-Armella et al., 2008), organize and analyze data (e.g., Konold et al., 2007), and investigate various content areas (e.g., Dick \& Hollerbrands, 2011).

Researchers have also investigated which fundamental characteristics of technology enhance students' understanding. Kaput (1992) argued that this would be possible because "the new electronic media now afford a whole new class of dynamic, interactive notations of virtually any kind" (p. 522). He stated that dynamic media make it easy to offer students a multitude of variations; interactive media generates an instance followed by a user's physical action. It could be argued that watching a video is more dynamic than reading a written a book because as video combines generic dynamic factors (e.g., digital, interactive, multimedia) to help students engage in the activity. However, dynamic mathematics requires a particular principle of dynamism. Jackiw and Sinclair (2009) proposed two principles: dynamism's mathematical aspect and pedagogical aspect. In dynamic mathematics software, mathematical representations are powerful and temporalized with continuity and continuous change (mathematical aspect) and embodied immediately with direct interaction (pedagogical aspect). For example, using a dynamic graphing tool (e.g., Desmos), a student explores the property of a slope in a linear graph by manipulating a slider. When the student moves the slider to the bigger number, the slope becomes steep closed to y-axis. When moving to the negative number, the direction of graph becomes opposite. Here, such visual representations of the graphs are dynamically changed (mathematical aspect) through the immediate interaction with the use of the slider (pedagogical aspect). 
Building on Kaput's foundation, Roschelle et al. (2017) asserted that such dynamism helps students attain a clear mathematical relationship as a new path to conceptual understanding. For example, geometry is a representative domain for dynamic mathematics software such as GSP (e.g., Hollebrands, 2007) and Cabri (e.g., Laborde, 1993). Through the use of dynamic geometry software (DGS), students can construct geometrical figures, measure lengths or angles of the figures, or manipulate elements of a figure that holds the same geometrical features. In addition to geometry, there are other content areas enhanced by dynamic representation, such as algebra (e.g., SimCalc; Roschelle et al., 2000) and statistics (e.g., TinkerPlots; Konold et al., 2007). However, there is few empirical studies about the meaningful use of technology in elementary mathematics classrooms compared to its use in secondary classrooms (Young et al., 2018). Some researchers extended the use of DGS for elementary students to support their understanding of geometrical concepts (e.g., Ng \& Sinclair, 2015) and early numbers (e.g., Sinclair \& Crespo, 2006). But these explorations are limited, applying in only a few specific content areas such as plane geometry.

\section{Developing Fraction Concepts With Digital Technology}

In the domain of fractions, several studies have used digital technology. Although hands-on manipulatives (e.g., fraction strips, pattern blocks) are more likely to be available to children in the classrooms, digital technology distinctively provides a powerful and flexible environment for them to represent fractions in different modes (Kaput, 1992). For example, Hunting et al. (1996) conducted a teaching experience with young children to investigate fraction learning and the role of their whole-number 
knowledge. They provided an operator-like technology called Copycat, which instantiated the fraction as an operator interpretation of rational numbers. Children were encouraged to determine which fraction was the appropriate numerical input and corresponding output or to determine what would be a possible input (or output) through the operation with selected fractions in the Copycat environment. This study showed that the technological environment could help stimulate and extend children's whole- and rational-number knowledge. Even though this study used discrete representations, such as dots, the children had measurement- and partition-based understandings of fractions.

Suh et al. (2005) also conducted a teaching experiment in a fifth-grade classroom through virtual fraction manipulatives from the National Library of Virtual Manipulatives (http://matti.usu.edu/nlvm/index.html). Also, the researchers used one applet from the NCTM online resources (https://www.nctm.org/Classroom-Resources/More-OnlineResources-from-NCTM/). The researchers examined students' learning of equivalent fractions (e.g., $5 / 7=10 / 14=15 / 21$ ) through circular representations. Students could manipulate up- and down-arrow buttons to change the number of partitions of the whole. When using the arrow buttons, students could explore multiple representations very quickly and make connections to symbolic representations. In addition, students could test their conjectures about patterns in fractions related to the changing number of pieces. Ultimately, this tool allowed fifth graders to identify a mathematical relationship related to factors and multiples in denominators and numerators.

Steffe and Olive (2002) designed JavaBars (Figure 5), which is software primarily aimed at examining the type of representations of fractions constructed by children and related understandings. Students could use menu buttons to operate and create bars of any 
size to represent a specific quantity through unitizing, segmenting, partitioning, and iterating. A physical model could not manipulate part of a partitioned whole, leaving the whole intact, whereas JavaBars enabled students to operationalize the part-to-whole relation (Olive \& Labato, 2008). A number of studies have used JavaBars to examine its effects on students' learning of fractions (Tirosh, 2000; Tzur, 1999), multiplicative relationships (Hackenberg, 2010), preservice teachers' fraction knowledge (Chinnappan, 2000), and teachers’ pedagogical content knowledge (Taylan \& da Ponte, 2016).

\section{Figure 5}

JavaBars Software

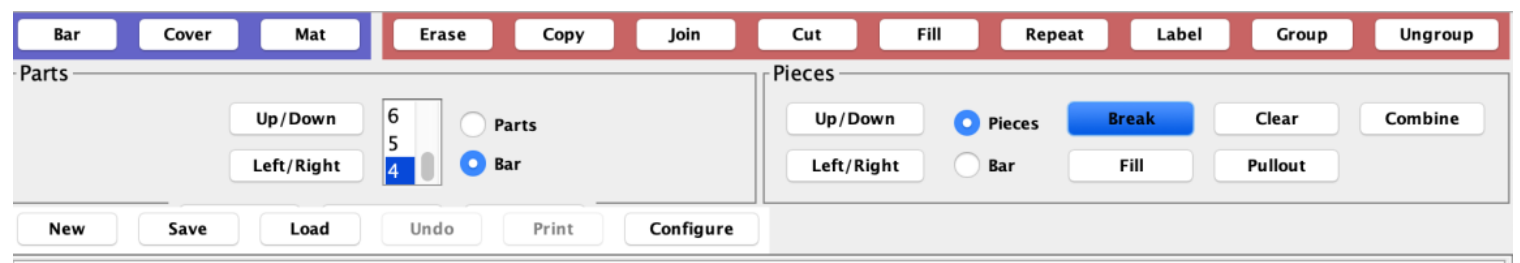
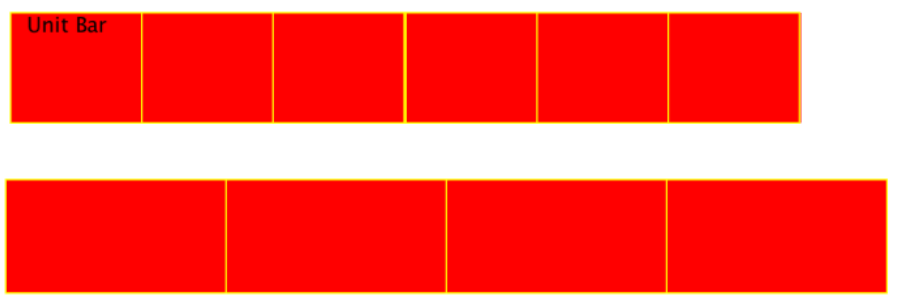

In terms of dynamic principles, JavaBars and other technologies seem to pertain to the pedagogical aspect of dynamic mathematics through immediate feedback; however, the methods of manipulating objects are less direct and less intuitive than the 
manipulations typical in the real world. When children use a physical tool (e.g., ruler or compass), they can directly operate and maneuver the tool with their own actions and intentions. However, the digital tools in those studies often required children to click menu buttons or to operate function keys to manipulate and generate objects on the screen. For example, in JavaBars, students often need to already decide the number of partitions they want in order to input the number. In this study, I provided a dynamic digital environment that allowed participants to manipulate representations directly through their actions (the pedagogical aspect of dynamism). In addition, I included continuous changes of representations (the mathematical aspect), which the previous studies lacked. From such continuous variation of mathematical objects, students have new opportunity to explore mathematical concepts more dynamically. The tool is described in detail in Chapter 3.

To sum up, students strive to understand fraction meaning with their prior knowledge even though fractions have an innate complexity. However, one critical problem with the current instructional approach seems to be the emphasis placed on the part-whole interpretation stemming from whole-number knowledge. It might reinforce students' limited understanding of fractions. An alternative approach would be to focus on measurement as a source of fraction concepts.

In this study, I devised a dynamic tool to examine children's thinking about fractions based on the measurement approach and investigated how elementary students engaged in a series of technology-based tasks. I also used this study's digital tool to help children extend their unit concept not only with prepartitioned measurement tools but also with flexible units that could vary continuously. The tool was mainly developed to 
support children's fraction understanding by allowing them to dynamically explore the relationships between quantities and units. The dynamic tool has the potential to provide a vehicle for students to understand fraction conceptions generally. Also, it presents an opportunity for students to experience something new about fractions by exploring the fundamental but long-neglected epistemological origin of fraction concepts: measurement.

However, more fundamentally, I wondered how children were influenced by the characteristics of this study's dynamic tool. What kinds of knowledge and skills would they draw on to solve tasks? How did they build up the meaning of fractions from the dynamic representations and mathematical actions provided in the dynamic tool's environment? Therefore, to understand how children develop the concepts of fraction as measure using digital technology, the theoretical framing in this study was instrumented mediation, which integrated the theories of instrumental genesis and semiotic mediation. This framework allowed me to see the tool's impact on fraction concepts, assessing both the technology and mathematical meaning at the same time. I could appreciate the whole picture of how students used and interpreted dynamic representations.

Through the comprehensive framework, I interrogated the interactions between children and the tool and the process of meaning making from the signs generated by the tool. With regard to the theory of instrumental genesis, I examined first how children appropriated the dynamic tool's different affordances or constraints when solving a series of tasks. Then I analyzed how they reasoned quantitatively between units and measures with the tool and drew mathematical knowledge (e.g., fractions, measurement). I also scrutinized how the use of the tool was connected to their understanding of fractions. 
Specifically, I delved into how the student produced visual, artifactual, and mathematical signs within a dynamic software program (e.g., long and short bars, fraction words) and how those signs contributed to making meanings related to fractions. This analysis provided insights into how students make sense of fraction concepts via the tool's dynamic representations. 


\section{CHAPTER 3: METHODOLOGY}

In this chapter, I describe how I gathered and analyzed data to answer the research questions. There are five sections in this chapter. The first section contains a description of and reflection on my pilot study, in which I tested a prototype of the tasks and tool used in the study. The second section is a description of the study's participants for this dissertation study. The third contains a description of the dynamic tool, including technological features and targeted mathematical content. The fourth describes the sources of the data collected. Finally, the last section explains the multiple rounds of analysis and the various analytical methods used in this study.

I employed an exploratory case-study method (Yin, 2014) to investigate children's understanding of fraction as measure. The case studies examined how understanding might change by using the dynamic tool developed for this study across a series of task-based clinical interviews. I hypothesized that the dynamic task would influence different types of strategy so that each solution was regarded as a unique case of mediated understanding. During the interviews, two kinds of data were collected primarily: transcribed explanations and screencast videos to track students' manipulations on the computer screen. The data were analyzed to identify both what kinds of mathematical ideas children have and how they use a dynamic tool.

\section{Pilot Study}

Prior to the dissertation study, I piloted a prototype of a digital tool and an interview protocol with an initial group of participants to gain insights about the tool's design and implementation, and also began to test an approach to data analysis (the first pilot: March 2019, the second pilot: April 2019). Because little research has been 
conducted on how children conceptualize the meaning of fraction as measure through the use of dynamic tools, it was crucial to investigate plausible ways to use the tool and to think about mathematical concepts prior to the main study.

From the first pilot study, I obtained feedback on how to revise the digital tools' prototype and the interview protocol, and I contemplated the best way to analyze the data. As a prototype, I had devised 10 tasks to examine children's basic measurement concepts (Tasks 1-3), their conceptualization of a fraction as a measure (Tasks 4-6), and fraction operations (Tasks 7-10). Young children, who did not have a formal instruction of fractions at schools, tried to solve all tasks during individual interview. After interviewing, I noted several structural and technical issues to be solved before implementing the main dissertation study. For example, in the first three tasks about measurement concepts, I designed the tasks to measure virtual objects with prepartitioned or size-adjustable rulers. When measuring a pencil (Task 1; Figure 6-left), some students initially estimated the length of the pencil in centimeters (" 4 centimeters"). When asked to use a prepartitioned ruler tool to measure the pencil, they did not manipulate the tool directly because they were not familiar with the tasks and the tool. When directed to use the tool, a second-grade student described one red segment of the ruler tool this way: "It was a half of the length of the pencil. So, two red strips." This particular interview informed me of the children's potential to internalize basic fractional terms from everyday situations (e.g., time and money contexts) or from other mathematics content areas (e.g., measuring length). Although this task contained the prepartitioned tool to measure the pencil with little cognitive demands, I noted that the children needed a 
tutorial before solving the main series of tasks so that they could become familiar with the tool's environment.

In addition, the first pilot study influenced the written prompt and interview protocol. For example, in Task 4 (Figure 6-right), the written prompt was "Measure the difference between length S and T through Dynamic Ruler." Corresponding to this prompt, children often utilized a measuring strategy with fractional units by counting the number of segments for each length and identifying the difference between two quantities. One second-grade student measured $\mathrm{S}$ and $\mathrm{T}$ with the red ruler tool. The student indicated the difference between $\mathrm{S}$ and $\mathrm{T}$ was four red segments. Then, the student was asked to measure $\mathrm{S}$ with $\mathrm{T}$ as a unit. This student pulled up the green ruler tool, adjusted a segment with the same length as T, and concluded three green segments were needed to measure S. Here, I noted the prompt was not direct enough to use $\mathrm{T}$ as the unit. What would be a better way to prompt children to demonstrate their understanding of fractions? I decided to ask more directive questions to focus on the meaning of a unit (T) and the measurement of a length (S) - e.g., "How many yellow rods (T) are needed to fit into the blue rod (S)?" Also, in the interview protocol, I included diverse questions to fully utilize the dynamic tool and to elicit the students' mathematical thinking relevant to fractions. For example, if a child did not adjust the size of a ruler, I would ask whether the child was able to change the size of the ruler to measure the given length. 


\section{Figure 6}

Tasks 1 and 2 in the First Pilot Study
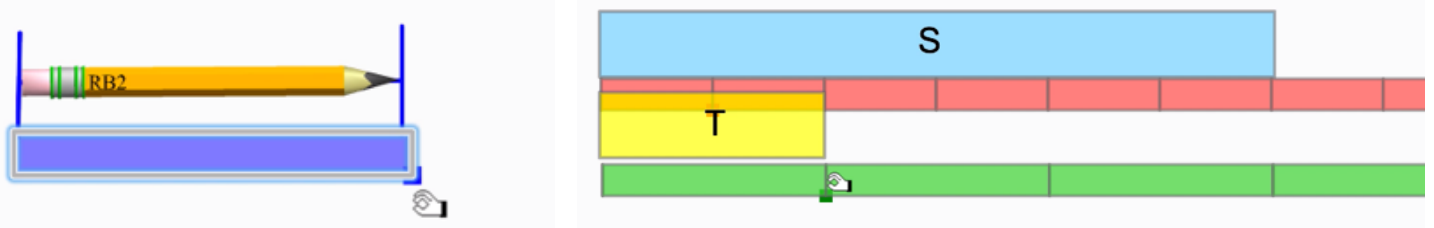

Prior to the second pilot study, I reduced the number of tasks to focus exclusively on how to conceptualize and develop children's concept of fraction as measure (what were Tasks 4 through 6 in the first pilot study). For that, I devised four tasks for each task series with different number combinations.

During the second pilot study, I further investigated interview methods by piloting interviews with a group of children and others with an individual child. In the case of the group of children, I recruited four third graders and interviewed with individual devices. I also recruited two third graders for individual interviews.

When observing the group interaction, it was interesting to see different interpretations of the same representations and actions with verbal exchanges. However, some students did not have a chance to explain how they approached a task and used the tool. Because my theoretical underpinning was related to the mediation process between mathematical words and the use of tools, I concluded that a group approach might not be ideal for collecting and subsequently analyzing how the students used words to describe their mathematical conceptualization. For this reason, for the main dissertation study, I chose to conduct interviews with individual children as they worked on a personal device. 


\section{Participants}

In this study, participants were recruited from a single school district in the Midwest region. From nine elementary schools, a total of 30 students participated in this study: 13 students in grade 3, eight in grade 4, and nine in grade 5. Potential participants were recruited through a digital flyer (https://peachjar.com/), and I interviewed individual kids after receiving the online consent from parents. In this study, because $43 \%$ of participants $(N=13)$ were English language learners (ELLs), it was more essential to reveal how their thinking when manipulating technology, even if their academic English was not perfect.

Children from multiple grade levels were recruited for maximum variation in test subjects (Table 1). This diversity provided a cross-sectional snapshot of how students thought about fractions when using the tool. After interviewing 30 students, I reached data saturation (Guest, Bunce, \& Johnson, 2006), as the remaining pool of potential subjects seemed to be generating strategies similar to those already analyzed. All participants were expected to have informal and formal knowledge of fractions drawn from everyday life and school mathematics (Mack, 2001). The duration of each one-time interview ranged from 45 to 81 minutes. The interviews were conducted after regular school hours in the school facility or outside of school to work with students' schedules. 


\section{Table 1}

Participants by Grade Levels

\begin{tabular}{ccc}
\hline Grade 3 $(\boldsymbol{N = \mathbf { 1 3 } )}$ & Grade $\mathbf{4}(\boldsymbol{N}=\mathbf{8})$ & Grade 5 $(\boldsymbol{N}=\mathbf{9})$ \\
\hline Brian & Anthony & Alex \\
Chansue & Arim & Darim \\
Emily & Charim & Gina \\
Fran & Eric & Hunter \\
Jack & Richard & Juli \\
Kijin & Sumin & Kein \\
Lauren & Sungho & Molly \\
Mary & Teo & Sandol \\
Minki & & Semi \\
Peter & & \\
Rogan & & \\
Sewoong & & \\
Youngjun & & \\
\hline
\end{tabular}

Note. All names are pseudonyms.

\section{Dynamic Ruler Tool}

In this study, I designed a series of tasks (see Appendix A) focused on building the mathematical structure to support students' understanding of fractions from measurement situations (Davydov \& Tsvetkovich, 1991; Dougherty, 2008). The tasks were designed to use the interactive software New Cabri (Laborde, 2016), which allows users to explore mathematical relationships and properties with interactive actions. I called this tool the Dynamic Ruler (Yeo, 2020).

\section{Dynamic Features of the Tool}

Like a physical ruler, this Dynamic Ruler was designed to measure a length displayed on a computer screen. What makes this tool dynamic? What is different from other manipulatives, such as fraction strips or Cuisenaire rods? Four interactive features were offered in the Dynamic Ruler environment (Figure 7): (a) adjusting the size of a 
unit, (b) moving a Dynamic Ruler, (c) trimming (or recovering) the number of pieces comprising a Dynamic Ruler, and (d) selecting other Dynamic Rulers from the menu of rulers. Each element is described in more detail in Figures 8-11.

\section{Figure 7}

\section{Screenshot of the Tutorial}

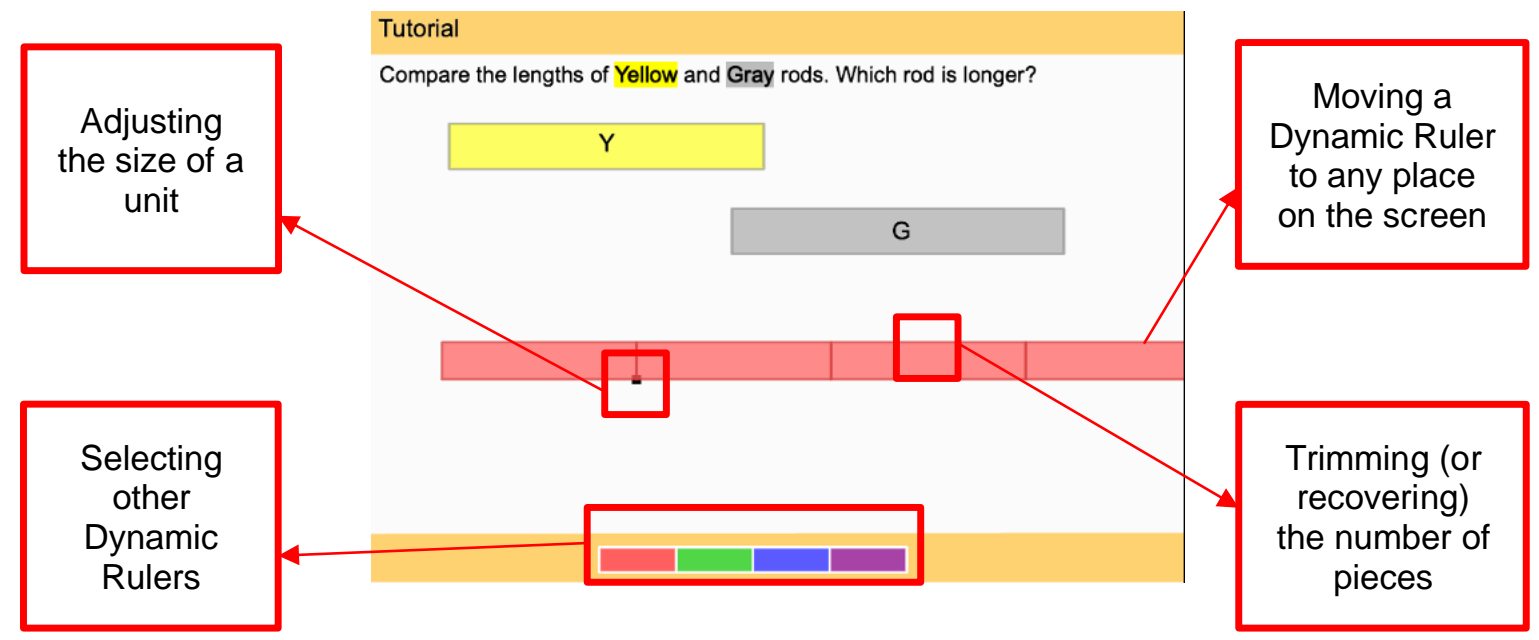

First, by adjusting the size of a unit by moving the designated point (Figure 8), all the units on the given ruler through dilation. For example, in Figure 8, the Blue Dynamic Ruler is below length Y and above G. All units on the Blue Dynamic Ruler are resized when the designated point is used to adjust the size of just one blue unit. Students could use this feature to explore various relationships between the measure and the units. The sequence of the study's tasks was also designed to encourage the eventual use of partial units to describe the multiplicative relationship between the unit and the measured quantity by adjusting the size of the units in the Dynamic Rulers. 


\section{Figure 8}

\section{Adjusting Feature}
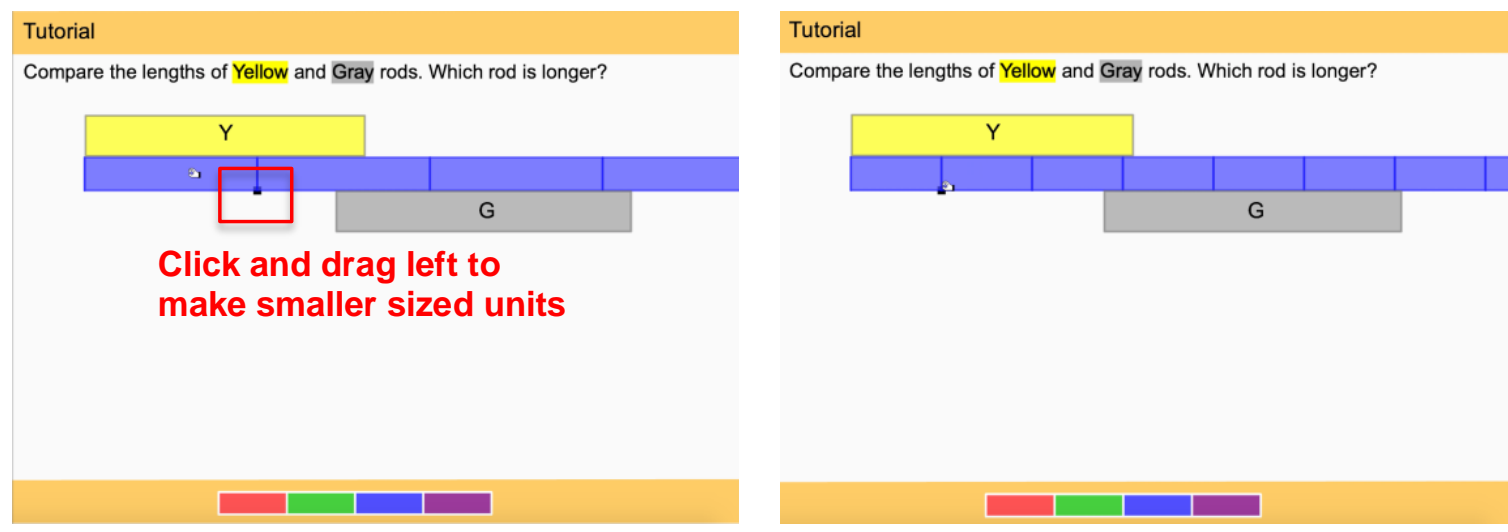

Second, students could move Dynamic Rulers from one place to another on the screen (Figure 9). They might measure a given length by putting a Dynamic Ruler next to an object. Because the color of a Dynamic Ruler was translucent, it was possible to place a Dynamic Ruler in the background of an object and overlap multiple Dynamic Rulers (Figure 9- right).

\section{Figure 9}

\section{Moving Feature}

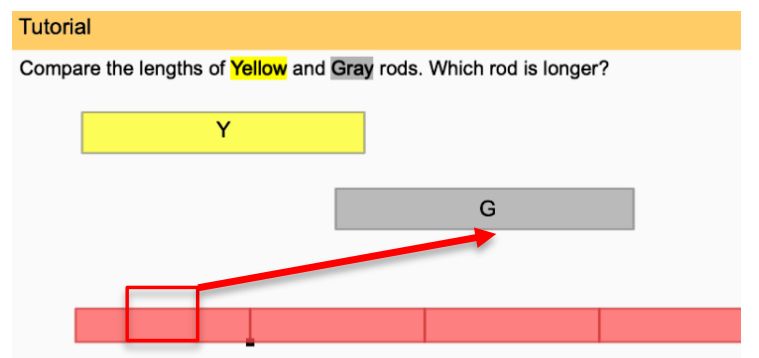

\section{Tutorial}

Compare the lengths of Yellow and Gray rods. Which rod is longer?

Click and drag

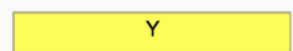

G

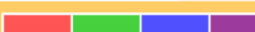

Note. The gray rod overlapped by the Red Dynamic Ruler, which is why the letter G appears in horizontal alignment with the ruler. 
Third, students could choose the number of pieces to display on the ruler by clicking a part of a Dynamic Ruler (Figure 10) to trim it. For example, when a student needed to represent exactly five pieces, the student could click the fifth piece, and then the segments to the right of the fifth one would disappear. By clicking the fifth one again, the rest of the Dynamic Ruler would appear once again.

\section{Figure 10}

\section{Trimming Feature}
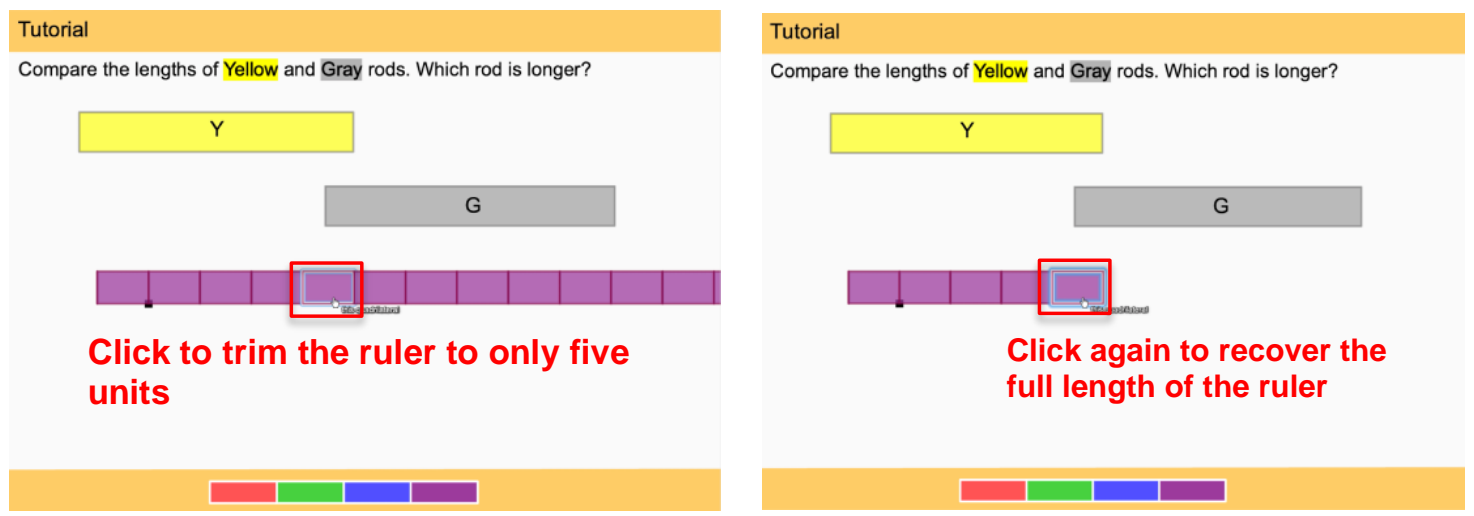

The fourth and final feature of the Dynamic Ruler environment was that a student could use multiple Dynamic Rulers (Figure 11) to compare units (or a unit and a part).

Students were empowered to decide for themselves if one Dynamic Ruler was enough to complete the given tasks. Students could choose from four Dynamic Rulers (red, green, blue, and purple) at the bottom of the screen and pull up whichever rulers they wanted whenever they wanted to use them. 


\section{Figure 11}

\section{Selecting Feature}
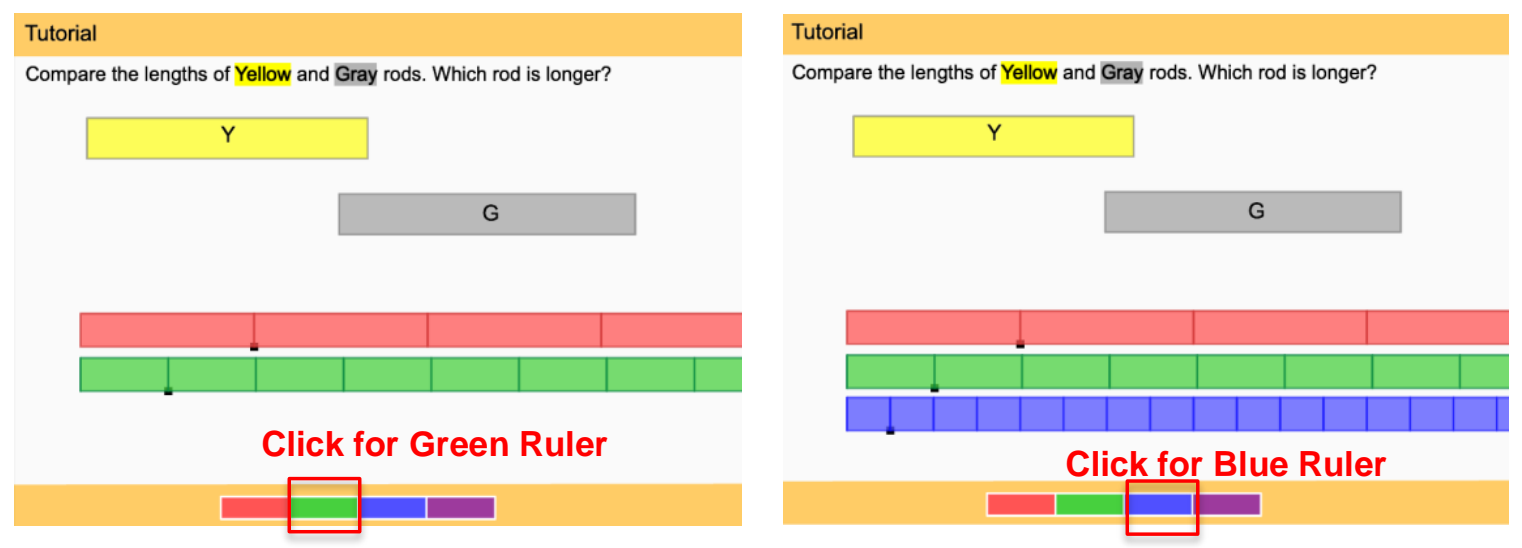

\section{Overview of the Tasks}

The tasks (Table 2) stemmed from prior research on the teaching and learning of fractions from a measurement approach (Davydov \& Tsvetkovich, 1991; Dougherty, 2008; Morris, 2000; Simon et al., 2018) and were refined through the two pilot studies with individual students as well as a group of students. I developed four task series, including the Tutorial Task (exploring major features of the tool: adjusting, moving, trimming, and selecting), Task Series 1 (using units to measure a length), Task Series 2 (using partial units to measure a length less than two whole units; the leftover part as a unit fraction of the given unit), and Task Series 3 (using partial units to measure a length greater than two whole units; the leftover part as non-unit fraction). Each task series consisted of four tasks. The first task in Task Series 1, Task Series 2, and Task Series 3 was an entry task and used an initially longer adjustable unit of the Dynamic Ruler. The second task in each of the series was more difficult in terms of the fractional part and encouraged students to use shorter adjustable units to explore different sizes of units compared to the first task. The third task in each series was an optional task for students 
who were struggling with previous tasks. It provided a pair of fixed rulers to ensure that students examined two possibilities when selecting Dynamic Rulers and considered the relationship between the two rulers. The fourth task in Task Series 1-3 used a longer adjustable unit and presented a more difficult ratio, such as one third.

\section{Table 2}

Summary of Fraction as Measure Tasks

\begin{tabular}{|c|c|c|c|}
\hline $\begin{array}{c}\text { Structure } \\
\text { of tasks within a } \\
\text { series } \\
\end{array}$ & Task Series 1 & Task Series 2 & Task Series 3 \\
\hline Prompt & \multicolumn{3}{|c|}{ How many $[$ unit] rods are needed to fit into the [quantity] rod? } \\
\hline Unit hierarchy & $\begin{array}{c}\text { Multiple whole } \\
\text { units }\end{array}$ & $\begin{array}{l}\text { Single whole unit }+ \\
\text { single partial unit }\end{array}$ & $\begin{array}{c}\text { Multiple whole units }+ \\
\text { multiple (or single) } \\
\text { partial units }\end{array}$ \\
\hline [quantity] : [unit] & Blue : Yellow & Orange : Brown & Pink : Gray \\
\hline $\begin{array}{l}\text { First Task } \\
\text { (default > unit) }\end{array}$ & 4 & 3 & 5 \\
\hline \multirow{2}{*}{$\begin{array}{l}\text { Second Task } \\
\text { (default < unit) }\end{array}$} & 3 & 5 & 11 \\
\hline & 1 & 4 & 4 \\
\hline \multirow{2}{*}{$\begin{array}{l}\text { Third Task } \\
(\text { default }=\text { fixed })\end{array}$} & 4 & 3 & \multirow{2}{*}{$\begin{array}{l}5 \\
2 \\
\end{array}$} \\
\hline & 1 & 2 & \\
\hline \multirow{2}{*}{$\begin{array}{l}\text { Fourth Task } \\
\text { (default > unit) }\end{array}$} & 5 & 4 & 8 \\
\hline & 1 & 3 & 3 \\
\hline
\end{tabular}

Note. The default size of the Dynamic Ruler varies by the task.

The tasks were designed with the idea of participatory and anticipatory stages (Simon et al., 2016; Tzur \& Simon, 2004). In the participatory stage, "learners develop an abstraction based on engagement in a particular activity" (Simon et al., 2016, p. 64). These activities include mental actions and physical manipulations. Students might 
develop mathematical knowledge but not need to possess established knowledge to anticipate the result. On the other hand, in the anticipatory stage, the learner can use the tool more purposefully. Prior to engagement with the tool, they might call upon the abstraction, then implement the abstraction with the tool. The distinction between the two stages is based on a reflective abstraction when learning mathematical concepts.

From this perspective, a student's work is defined by the situation in a sequence of tasks and by the given tool's environment, rather than by a written prompt on a paper or screen. The participatory stage might not require students to use relevant abstractions that were cued by a specific task situation. For example, Task 2-1 (Figure 12-top) begins by asking the user to treat the bottom rod as a unit size and to determine how many units are needed to measure the longer rod: "How many orange rods are needed to fit into the brown rod?" Students were asked to use the Green Dynamic Ruler (or other Dynamic Rulers) to figure out the relationship between the orange and brown rods. Students might learn by trial and error that they can make a Dynamic Ruler with units that approximate the length of the orange rod and then use that ruler to measure the brown rod. Furthermore, they might continue to explore and discover that they can use partitions of the orange rod to measure the brown rod. Because they did not start by strategically creating equipartitions of the orange rod, we would consider their work to be in the participatory stage; they were playing within the environment without a specific plan in might.

On the other hand, the anticipatory stage uses more general questions without a hint of how to solve the tasks. Students working in the anticipatory stage must summon a mathematical abstraction before they use the dynamic tool. Building on the previous 
example, once successfully employed in Task 2-1, students might begin to use anticipatory reasoning by applying the same strategy on subsequent tasks, strategically testing different partitions of the given unit to measure the given length. I designed specific features of the Dynamic Ruler environment to encourage students to develop various strategies as they used the tool. For example, students could use optional fixed rulers (Task 2-3, Figure 12-middle), which prompted students to measure lengths using units that are already formed as equipartitions of the given unit. In these tasks, they might develop and solidify the idea of equipartitioning the unit (participatory) in a way that could be applied to later tasks (anticipatory). In Task 2-4 (Figure 12-bottom), students might try to use the knowledge gained from the previous task to solve the length measurement task with anticipatory reasoning. However, if the previous strategy (or knowledge) did not work, students could return to the participatory stage by exploring other possible ways to solve the task.

\section{Figure 12}

Examples of Task in Series 2 (2-1, 2-3, and 2-4)

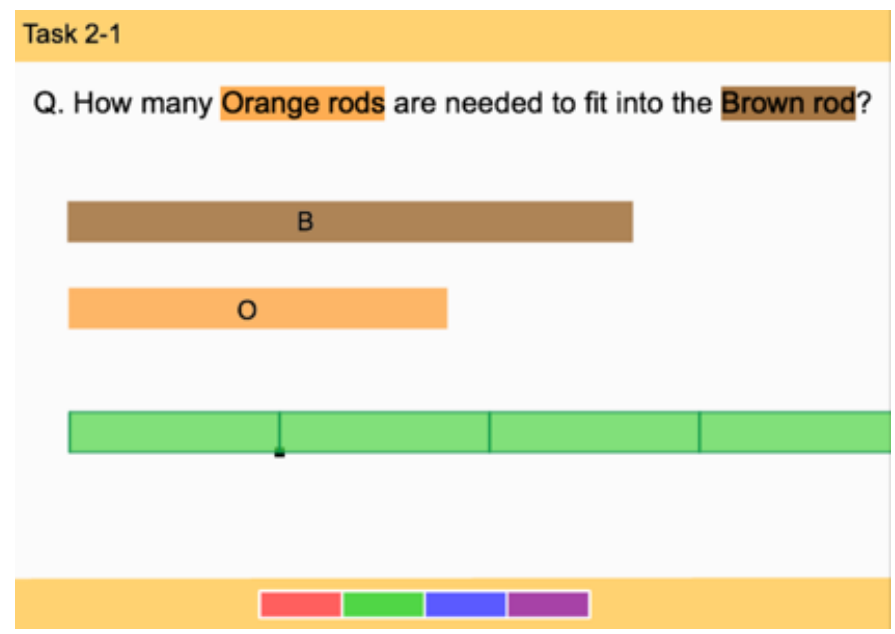




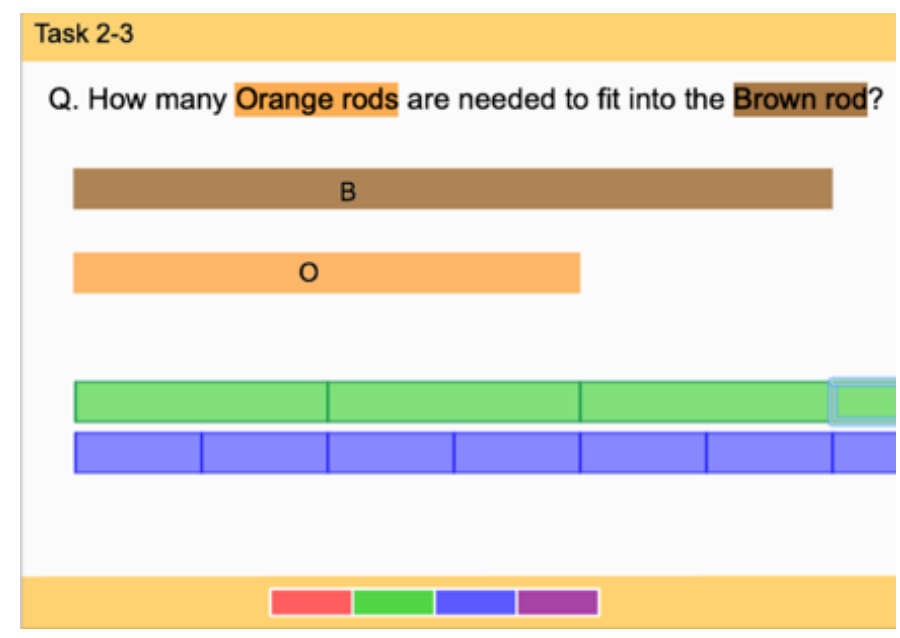

Task 2-4

Q. How many Orange rods are needed to fit into the Brown rod?

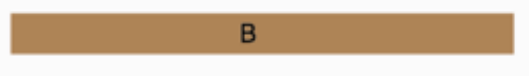

o

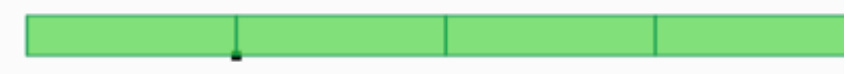

Before delving into each task series, I describe the meaning of key terms related to units and measurement in the fraction-as-measurement tasks (Figure 13). A quantity is a linear object to be measured which is static and placed on the top of the screen (pink rod). A given original unit refers to the unit-sized rod which is also static and placed under the quantity (gray rod). A leftover refers to a remaining length that does not equal a whole given unit (the length difference between the pink and gray rods) - i.e., it is too short or too long to measure with exactly a given unit. A partial unit refers to a scaled unit length (a piece of the red ruler). This partial unit could measure a quantity as well as a given original unit. An intermediate unit refers to a unit for measuring a leftover part 
only (a piece of the green ruler). Students can generate a given-sized original unit, a partial unit, an intermediate unit with the Dynamic Ruler tool as measuring units (a blue piece, a red piece, a green piece).

\section{Figure 13}

Key Terms in Fraction-as-Measurement Tasks

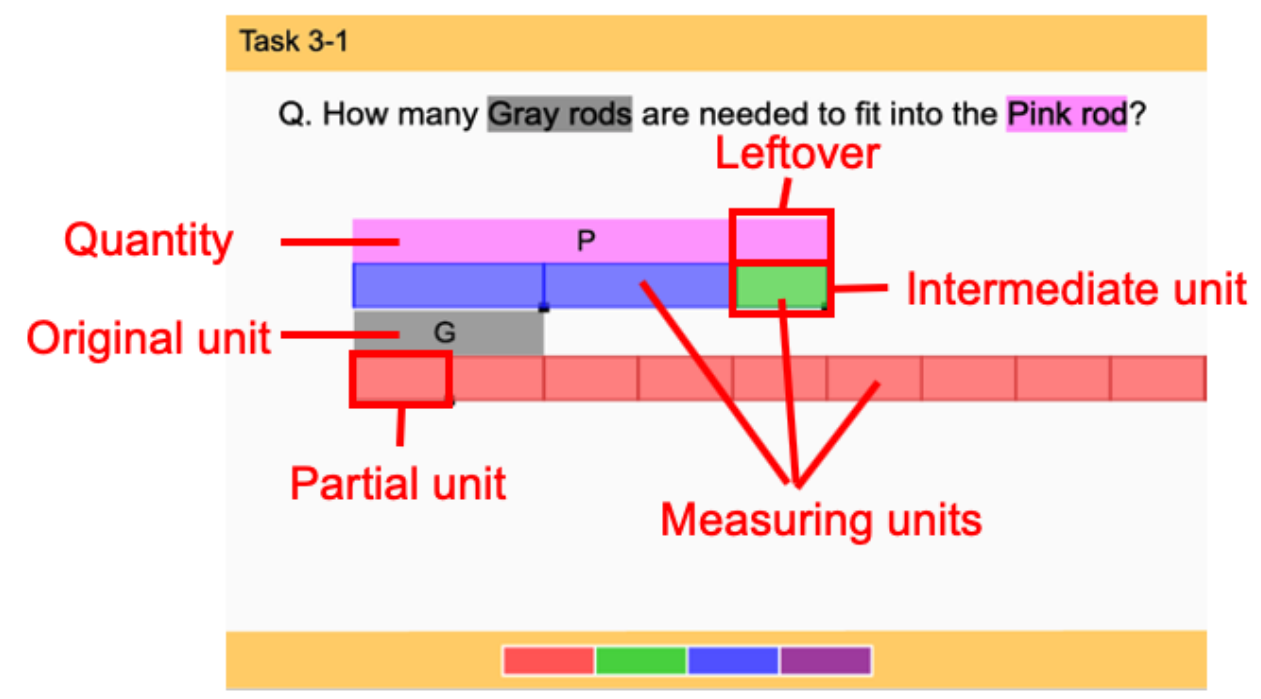

Drawing upon participatory and anticipatory ideas, I developed four tasks series for task-based interviews: Tutorial, Task Series 1, Task Series 2, and Task Series 3. In the Tutorial Task, children could explore the tool freely at first (Figure 14). Because the yellow and the gray rods were static, students had to use the Red Dynamic Ruler for the comparison. If they did not recognize the interactive features of the tool (adjusting, moving, trimming, and selecting), I indirectly asked them to use some of these features by questioning (e.g., If the student did not independently realize that they could adjust the units on a Dynamic Ruler, I asked, "What does the black point do on the Dynamic 
Ruler?"). In addition, the Tutorial Task mathematically involved the concept of informal measurement by using nonstandard units.

\section{Figure 14}

Tutorial Task

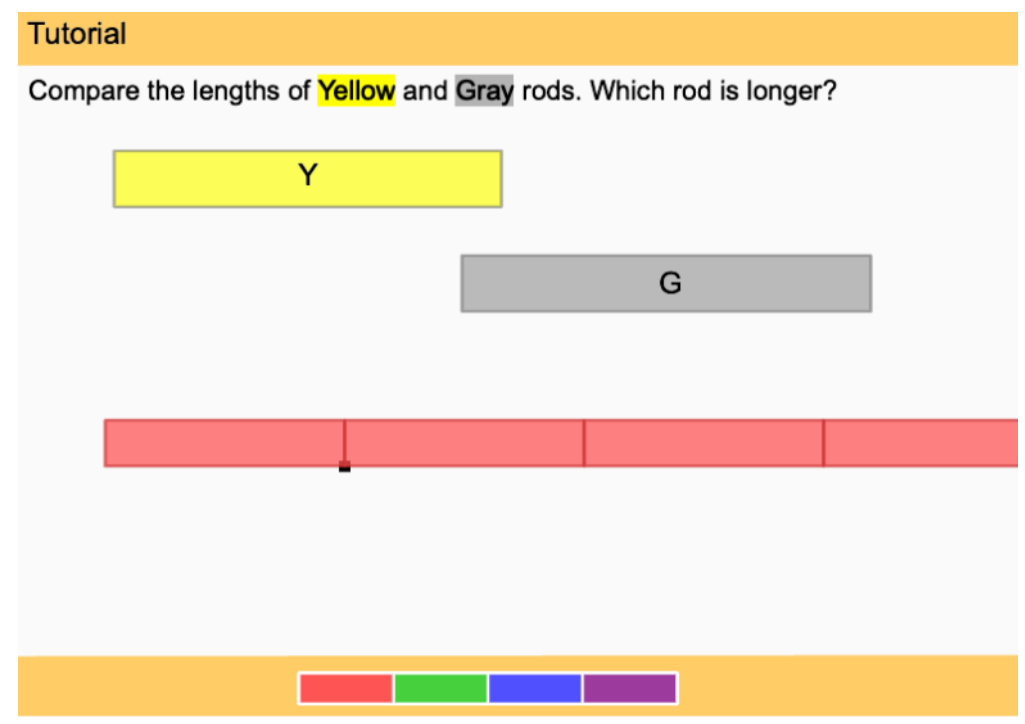

In Task Series 1 , the children were asked to measure the linear quantity with the given unit length to elicit a fundamental idea of measuring length (Figure 15). If they unitized the yellow rod, the measure was a whole-number result with no leftover length. Aligning and matching the Dynamic Ruler with the bottom yellow rod in Figure 15, the students might have counted the number of the pieces by indicating pieces with their mouse cursor or using skip counting. In so doing, students could recognize that the number obtained from length measurement was dependent on the size of the unit. This approach gave the students a chance to reason that the smaller the unit used to measure, the larger the number of the units needed for the measure. The ratio between the two rods 
in each task varied: the ratio in Task 1-1 was 4:1; Task 1-2, 3:1; Task 1-3, 4:1; and Task 1-4, 5:1.

\section{Figure 15}

Task Series 1

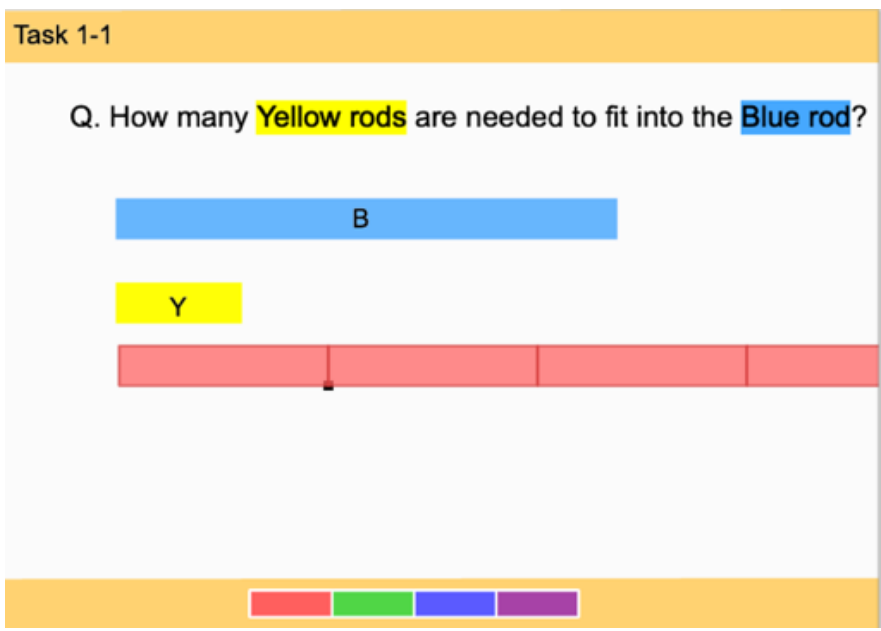

Task 1-2

Q. How many Yellow rods are needed to fit into the Blue rod?

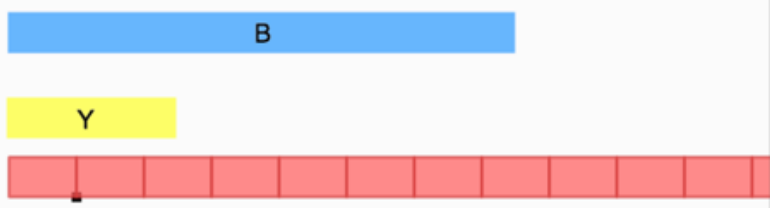




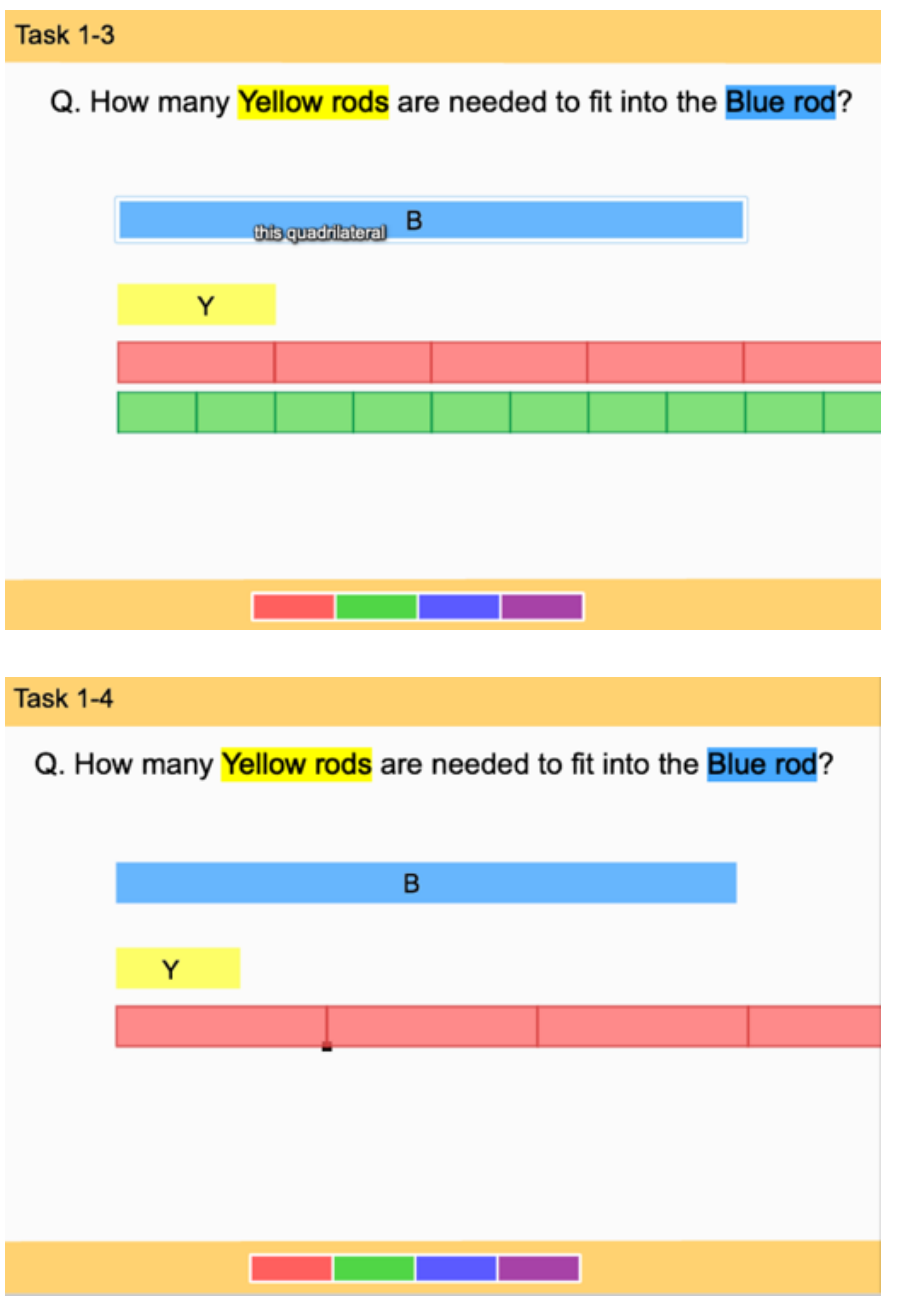

In Task Series 2, children were supposed to use partial units to measure a length, and the measure was less than two whole units of the given unit with the leftover as a unit fraction $(1 / n)$ of the given unit (e.g., the orange rod in Figure 16). Students might have known easy fractional terms before this section (e.g., a half). However, the tasks were designed to elicit more complicated fractional amounts and to represent a difference between two quantities by reasoning multiplicatively. Students were required to use a new unit to measure the leftover length between two quantities (the orange and brown rods). When measuring the longer rod with the shorter rod as the given unit, it was insufficient to use whole-number multiples of the shorter rod because a leftover length 
remained when the students attempted to fit one or more whole "short-rod units" into the longer rod. This feature of the tasks pressed students to make unit relations between the given units of measure and the leftover parts. The ratio between two rods varied by task: the ratio in Task 2-1 was 3:2; Task 2-2, 5:4; Task 2-3, 3:2; and Task 2-4, 4:3.

In solving Task Series 2, students were encouraged to think about the relationship between the given unit length defined in each question (e.g., the shorter orange rod in Figure 16), the quantity to be measured (the longer brown rod), and the new unit (the leftover). For example, in Task 2-1, students could use a mixed number (e.g., 11/2) or improper fractions (e.g., $3 / 2$ ), or to describe the difference in terms of whole-number ratios (e.g., "It takes two blues to measure the orange rod and three blues to measure the brown rod.”). Whenever students use the fractional terms, I asked them to clarify the referent unit for the fractions (e.g., "Half of what?") and where the fractional terms came from.

\section{Figure 16}

Task Series 2

Q. How many Orange rods are needed to fit into the Brown rod?

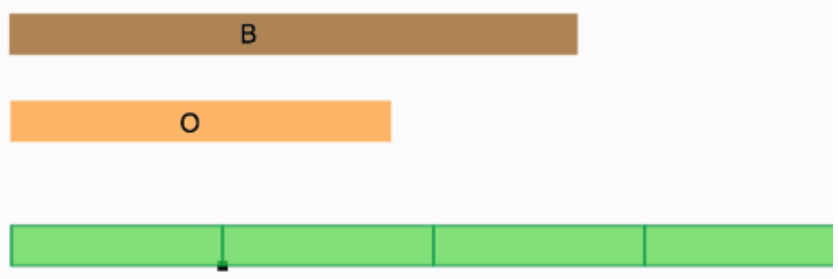


Task 2-2

Q. How many Orange rods are needed to fit into the Brown rod?
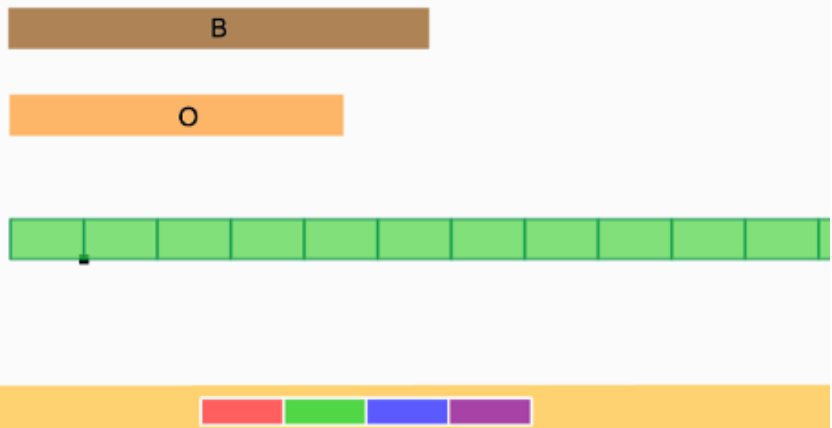

Task 2-3

Q. How many Orange rods are needed to fit into the Brown rod?

B

○
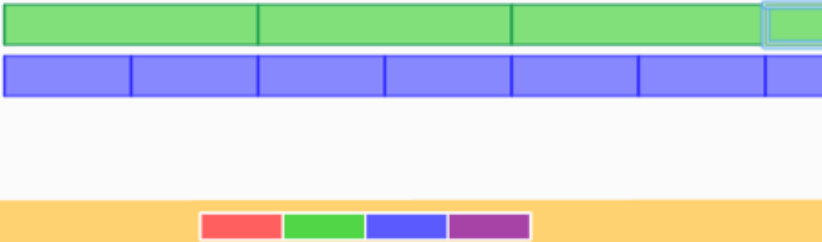

Task 2-4

Q. How many Orange rods are needed to fit into the Brown rod?

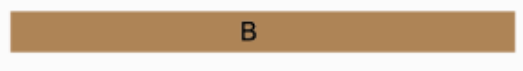

O
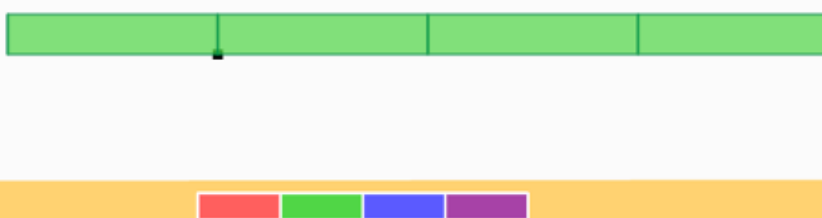

The last set of tasks in the Dynamic Ruler environment comprised Task Series 3, which challenged students to use partial units to measure a length. Each correct answer 
was greater than two whole units with the leftover as a non-unit fraction $(\mathrm{n} / \mathrm{m})$ of the given unit (Figure 17). Task Series 3 was an extended version of Task Series 2 in many ways. The most striking difference between the two series centered on the ratio between the two lengths in each task. The ratios increased the difficulty type of determining the correct length when compared to Task Series 2. From a perspective of iterating multiple units (Steffe \& Olive, 2010), Task Series 3 might seem easier than the previous series. However, using the Dynamic Ruler tool is not the same as using unit iteration because of the Dynamic Ruler's adjusting feature. As one of the main interactive features of a Dynamic Ruler, children are able to adjust the size of one of the ruler's pieces and, thus, simultaneously adjust the size of the rest of its pieces.

In addition, the leftover lengths in Task Series 3 were not easy to estimate by merely eyeballing the differences in the rods' lengths because the leftover part was not a unit fraction that equipartitioned the given unit. In Task Series 2, the given unit length (e.g., the orange rod in Figure 17) might not directly measure the requested quantity (brown rod) through unit iteration. Still, children could measure both rods with the leftover as a new partial unit (the leftover equals half of the orange rod; the orange rod equals two halves; the brown rod is $3 / 2$ ). On the other hand, the leftovers in Task Series 3 made it hard to estimate a fractional amount and were even longer than the gray rod (see Figure 17; e.g., the leftover part is $3 / 2$ of the gray rod). Therefore, there was the potential that the students might not measure any rod using only the given unit length. These characteristics of the tasks pushed students to try different partitions and to relate the leftovers and the given unit lengths by finding new partial-measure units. 
If a student struggled with the initial task in Task Series 3, then I had the option of skipping to Task 3-3, which included a predetermined partial-measure unit that equaled the leftover length. By presenting a ruler at the beginning of the task that equaled the leftover length, Task 3-3 lessened the students' cognitive load and allowed them to make connections between the given unit length, the available Dynamic Rulers, and the longer rod that the students must measure. The ratios between the two rods in each task were more complicated than Task Series 2: Task 3-1 (5:2), Task 3-2 (11:4), Task 3-3 (5:2), and Task 3-4 (8:3).

\section{Figure 17}

Task Series 3

Task 3-1

Q. How many Gray rods are needed to fit into the Pink rod?

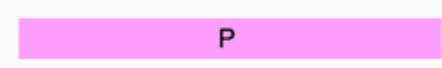

G

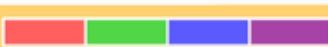


Task 3-2

Q. How many Gray rods are needed to fit into the Pink rod?

$$
\text { P }
$$

G
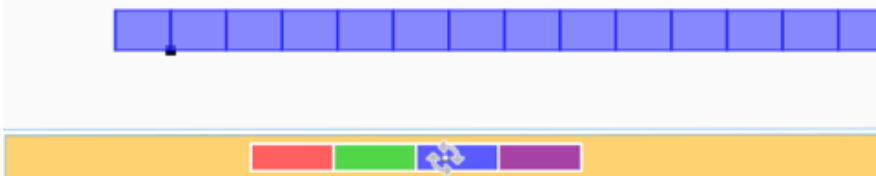

Task 3-3

Q. How many Gray rods are needed to fit into the Pink rod?

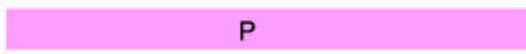

G
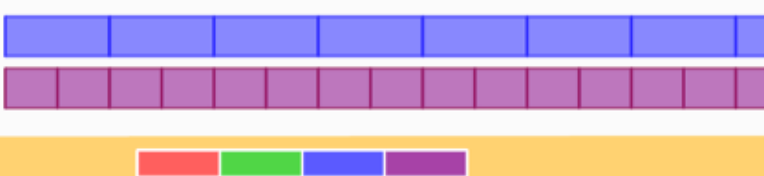

Task 3-4

Q. How many Gray rods are needed to fit into the Pink rod?

\section{$P$}

G

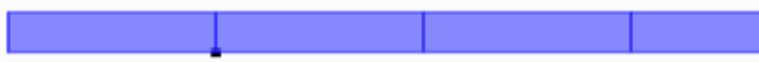

\begin{tabular}{|l|l|l|}
\hline & 口 \\
\hline
\end{tabular} 


\section{Data Sources}

In this study, task-based clinical interviews (Ginsburg, 1997) were conducted with individual students during their one-time interviews. Following the suggestions of Ginsburg (1997), I delved into students' understanding by requiring reflection, asking clarifying questions, and challenging the students with extending questions. In addition, the follow-up questions were nondirective, and the interview protocol (Appendix A) included as many contingencies as possible (Goldin, 1997). These contingencies were mainly drawn from the two pilot studies.

As a primary data source, each interview was recorded by a screencasting tool (QuickTime Player) and transcribed to track how individual students interacted with the dynamic tool when they were engaged in the tasks. During the transcription of the video, I tentatively marked instances that seemed to involve mathematical words relevant to fraction concepts as well as mediated understanding through the use of the dynamic tool. I used MaxQDA2020 software to include the time dimension in transcriptions, which allows for every single statement to be transcribed with the exact time connected to the recorded videos (Figures 18 and 19). I also collected field notes throughout the interviews, focusing on students' potential to understand the tasks and on their nonverbal expressions. 


\section{Figure 18}

\section{Example of Coding Video Data}

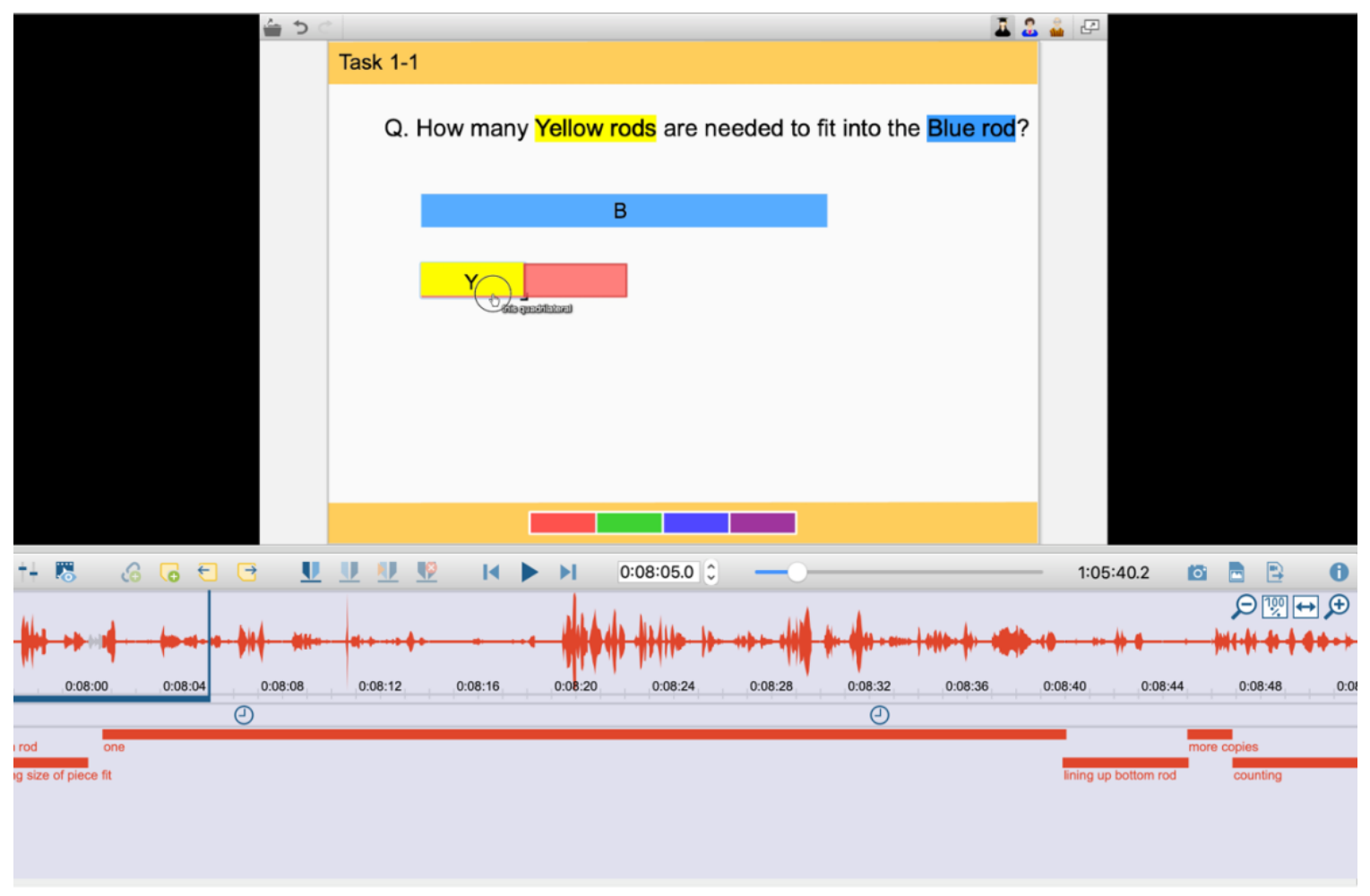

\section{Figure 19}

\section{Example of Coding Transcription}

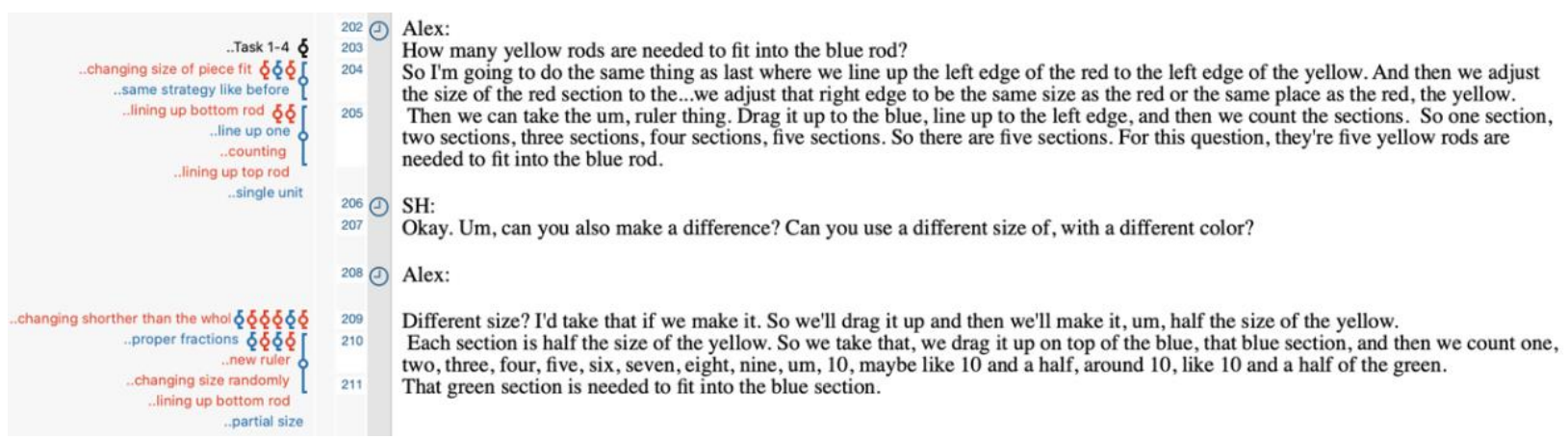

\section{Data Analysis}

I conducted multiple phases of analysis in order to focus on two major aspects of the data, mathematical actions and mathematical ideas. Mathematical actions are related 
to an observable part of how children solve a given task involving the instruments.

During the children's initial activity, I paid attention to their purposeful actions to explore the Dynamic Ruler tool and to manipulate the tool in approaching the measurement tasks. The mathematical actions are varied by the users' previous knowledge (instrumentalization) and influenced by affordances and limitation of the tool (instrumentation) (Trouche, 2004). Mathematical ideas represented mathematical objects in discourse (e.g., fractions, measurement concepts) and connoted mathematical thinking related to the concept of fraction as measure. After the initial exploration of the Dynamic Ruler tool, children were asked to describe their strategy to solve the tasks with verbal explanations. This activity invites them to produce their personal artifact signs and mathematical signs (Bartolini Bussi \& Mariotti, 2008). Because the artifact signs use their reference to their artifact directly and relate to mathematical meanings, they are centralizing on the use of the artifact and contributing to the process of meaning making. The mathematical signs are related to mathematical meaning as commonly shared in school mathematics and including mathematical terminology, definitions, and properties. In the following section, I describe the three phases of the data analysis: the iterative process of coding, the development of a framework about strategy types, and the design of conceptual profiling.

\section{Coding Scheme}

I used iterative methods of coding (Miles et al., 2013) in this study. In the first phase of coding, I analyzed interview data in terms of mathematical actions and mathematical ideas with open coding (Corbin \& Strauss, 2015). In the second phase, I employed axial coding by analyzing episodes of coding cooccurrences, and I identified a 
relationship between mathematical actions and ideas. This iterative coding was a cyclic process of questioning and reflecting on the data while comparing and refining categories and subcategories.

In the first phase of coding, I watched videos of the interviews several times. Then I coded each student's interview data to categorize their understanding of fractions while they used the Dynamic Ruler. The categories were based on an initial coding scheme (Appendix B) that was drawn from relevant literature and were as follows: fraction and assigning a number to a quantity (Webel \& Deleeuw, 2016), unit coordination (Steffe, 1992), partitioning (Confrey, 1994; Empson, 1999), additive reasoning (Carpenter et al., 2014), multiplicative reasoning (Thompson \& Saldanha, 2003), and clicking and dragging (Hollebrands, 2007).

The coding scheme was iteratively refined by reviewing transcripts of the students' tasks and by assessing the actual coding process. The final coding scheme consisted of mathematical actions and mathematical ideas. For the mathematical actions, I used process coding, using gerunds to present actions in the data to look for ongoing actions and interactions as responses to situations or problems. I used descriptive coding for the mathematical ideas. It "summarizes in a word or short phrase - most often as a noun - the basic topic of a passage of qualitative data" (Saldaña, 2016, p. 88). In Table 3, I briefly describe the categories, subcategories, and codes of the refined final coding scheme. 


\section{Table 3}

Refined Final Coding Scheme

\begin{tabular}{|c|c|c|}
\hline Category & Subcategory & Codes and description \\
\hline \multirow{6}{*}{$\begin{array}{l}\text { Mathematical } \\
\text { actions }\end{array}$} & \multirow[t]{3}{*}{ Hovering } & Counting ones: counting one by one \\
\hline & & Grouping: clustering a set of units \\
\hline & & $\begin{array}{l}\text { Indicating the edges: moving vertically to } \\
\text { check the alignment between edges }\end{array}$ \\
\hline & \multirow[t]{3}{*}{ Dragging } & $\begin{array}{l}\text { Lining up: positioning the Dynamic Ruler to } \\
\text { align the edges of the rods (lining up one of } \\
\text { two rods, lining up both rods) }\end{array}$ \\
\hline & & $\begin{array}{l}\text { Changing size: adjusting the size of pieces of } \\
\text { the Dynamic Ruler by dragging the black } \\
\text { dot (making the piece longer or shorter than } \\
\text { the default size, adjusting) }\end{array}$ \\
\hline & & $\begin{array}{l}\text { Commeasuring: measuring the given unit } \\
\text { length and quantities at the same time }\end{array}$ \\
\hline \multirow[t]{6}{*}{$\begin{array}{l}\text { Mathematical } \\
\text { ideas }\end{array}$} & \multirow[t]{3}{*}{ Unit } & $\begin{array}{l}\text { Unitizing: assigning a unit to measure a given } \\
\text { length (whole, double, partial, random) }\end{array}$ \\
\hline & & $\begin{array}{l}\text { Unit relation: relationships between units (two } \\
\text { units, three units) }\end{array}$ \\
\hline & & $\begin{array}{l}\text { Partitioning: partitioning the object by the unit } \\
\text { (partitioning objects; } n \text { parts make a whole) }\end{array}$ \\
\hline & \multirow[t]{3}{*}{ Fractions } & $\begin{array}{l}\text { Fraction notation: the way to express } \\
\text { fractional values (proper fraction, improper } \\
\text { fraction, mixed number, decimal) }\end{array}$ \\
\hline & & $\begin{array}{l}\text { Equivalent fractions: recognizing the same } \\
\text { value between different notations } \\
\text { (equivalence between notations, same size) }\end{array}$ \\
\hline & & $\begin{array}{l}\text { Fraction operations: constructing fractions } \\
\text { based on various operations (additive, } \\
\text { multiplicative, proportional) }\end{array}$ \\
\hline
\end{tabular}




\begin{tabular}{|c|c|c|}
\hline Category & Subcategory & Codes and description \\
\hline & $\begin{array}{l}\text { Fraction as } \\
\text { measure }\end{array}$ & $\begin{array}{l}\text { Unit fraction: identifying one segment as } 1 / n \text {th } \\
\text { (halving; } n \text { of } n \text {th }=1 \text { ) }\end{array}$ \\
\hline & & $\begin{array}{l}\text { Unit coordination: assigning the selected unit } \\
\text { length to measure the given length (using a } \\
\text { singleton unit, composite unit) }\end{array}$ \\
\hline
\end{tabular}

As shown in Table 3, the mathematical actions category focused on the purposeful use of technological tools: hovering and dragging. Students' hovering of the mouse cursor was related to counting the pieces of a Dynamic Ruler one by one, grouping them, and checking the alignment between rods. First, children moved the mouse cursor to count the number of pieces of the Dynamic Ruler (e.g., "So that's one, two, three, four, five, six, seven, eight."; Figure 20). Hovering on each piece of the Dynamic Ruler, they drew a circle or a check-mark with the mouse cursor to figure out the number of the pieces.

\section{Figure 20}

Hovering: Counting One by One

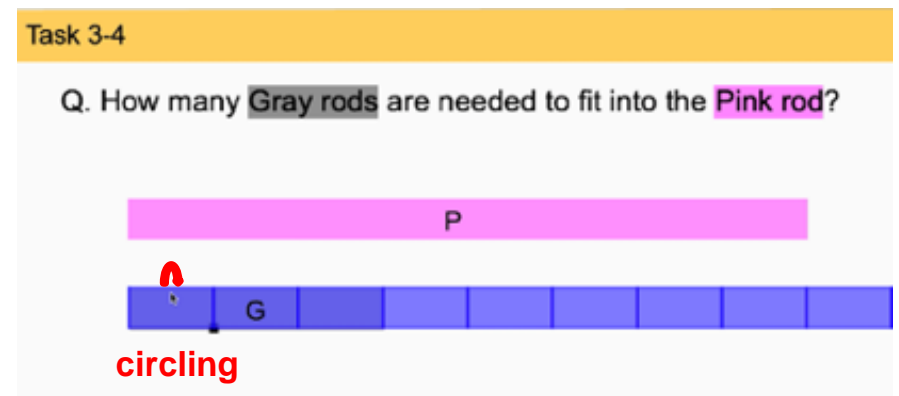



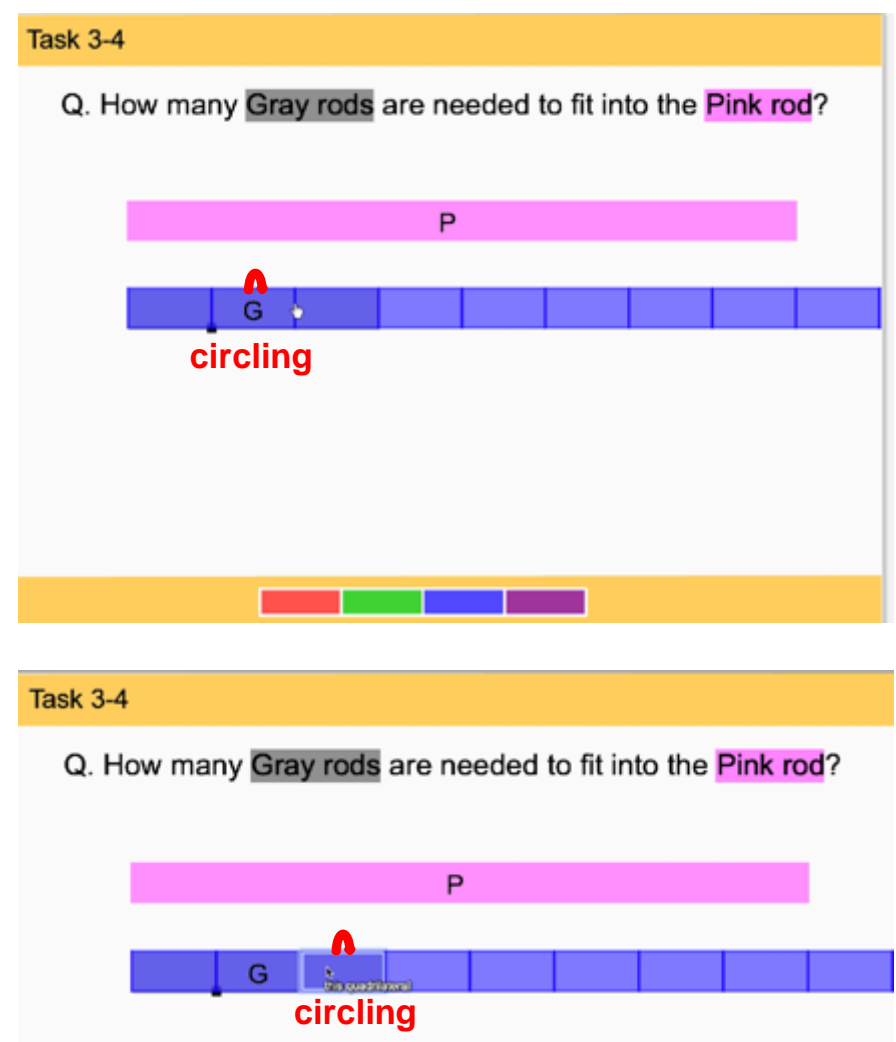

Note. The gray rod overlapped by the Blue Dynamic Ruler, which is why the letter G appears in horizontal alignment with the ruler.

Second, when counting the number of pieces, they moved the cursor to form a circular shape to group the pieces (e.g., "Three of the thirds, that's equal to one [of] the oranges."; Figure 21). One group of pieces that a student circled was often the same as the one whole unit. 
Figure 21

Hovering: Grouping

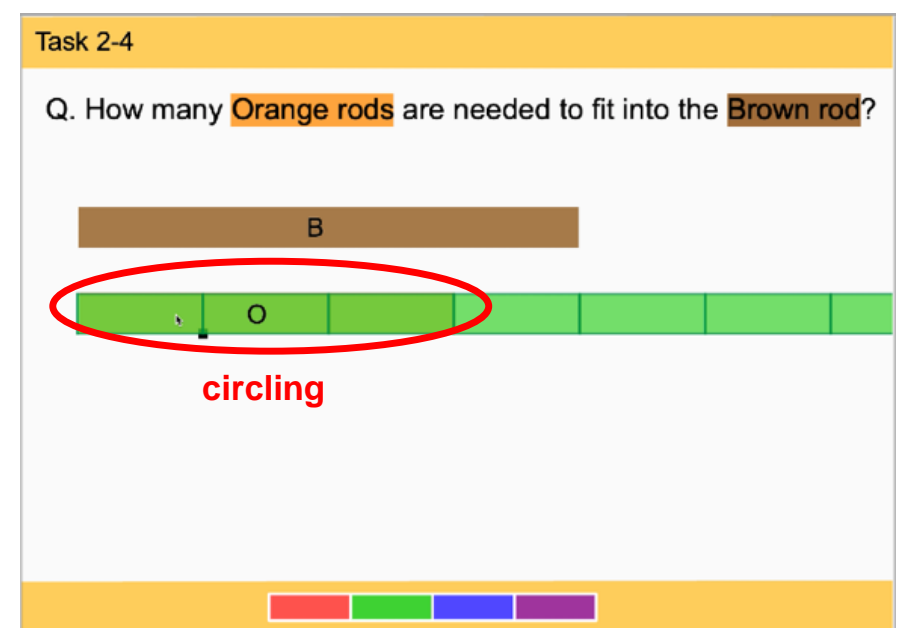

Task 2-4

Q. How many Orange rods are needed to fit into the Brown rod?
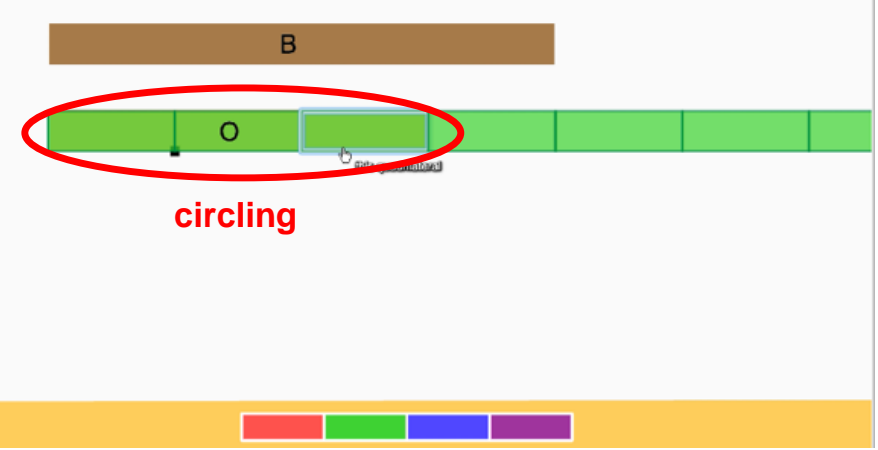

Note. The orange rod overlapped by the Green Dynamic Ruler, which is why the letter O appears in horizontal alignment with the ruler.

Third, the children moved the mouse cursor vertically to check whether one edge of the longer rod was matched with the Dynamic Rulers' pieces (e.g., "There's an edge that lines up."; Figure 22). 


\section{Figure 22}

Hovering: Indicating the Edges

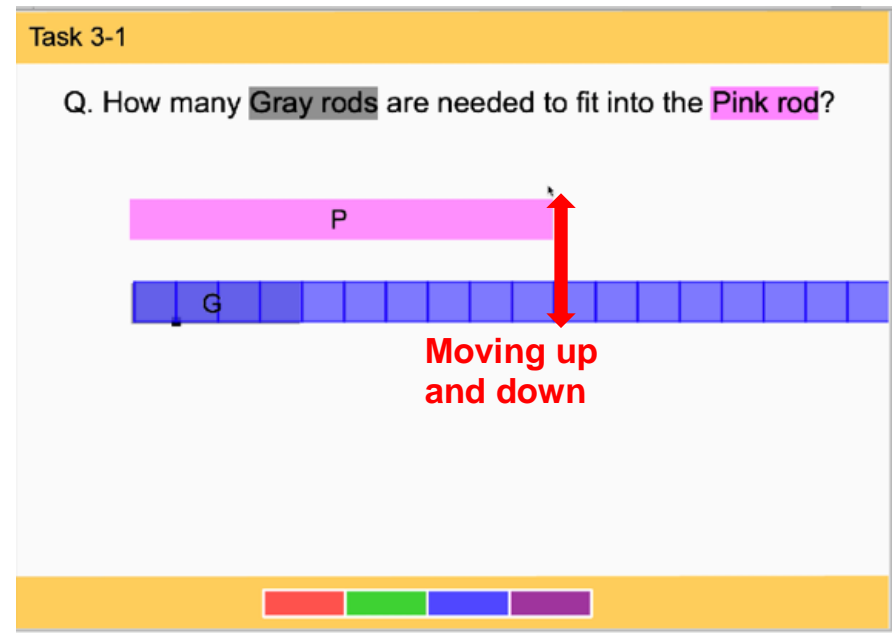

Task 3-1

Q. How many Gray rods are needed to fit into the Pink rod?

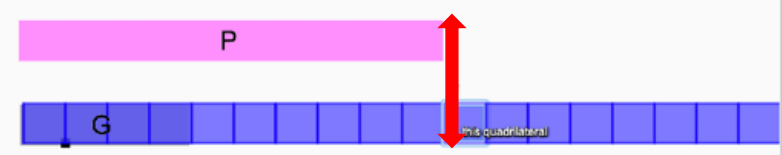

Moving up

and down

Note. The gray rod overlapped by the Blue Dynamic Ruler, which is why the letter G appears in horizontal alignment with the ruler.

Student's dragging involved lining up Dynamic Rulers related to units or quantities, changing the size of one piece of a Dynamic Ruler, and measuring the given unit length and quantities at the same time (commeasuring).

First, children dragged the Dynamic Ruler over the bottom rod and the top rod (or to the blank middle space between the two rods) to line up the ruler with rods (e.g., "So, 
if we move it up, there are five of the blue sections to fit into the pink rod."; Figure 23). The students performed a similar action when they placed the zero unit mark on the ruler at one end of the object to be measured to facilitate measuring the length of the object.

Figure 23

Dragging: Lining Up
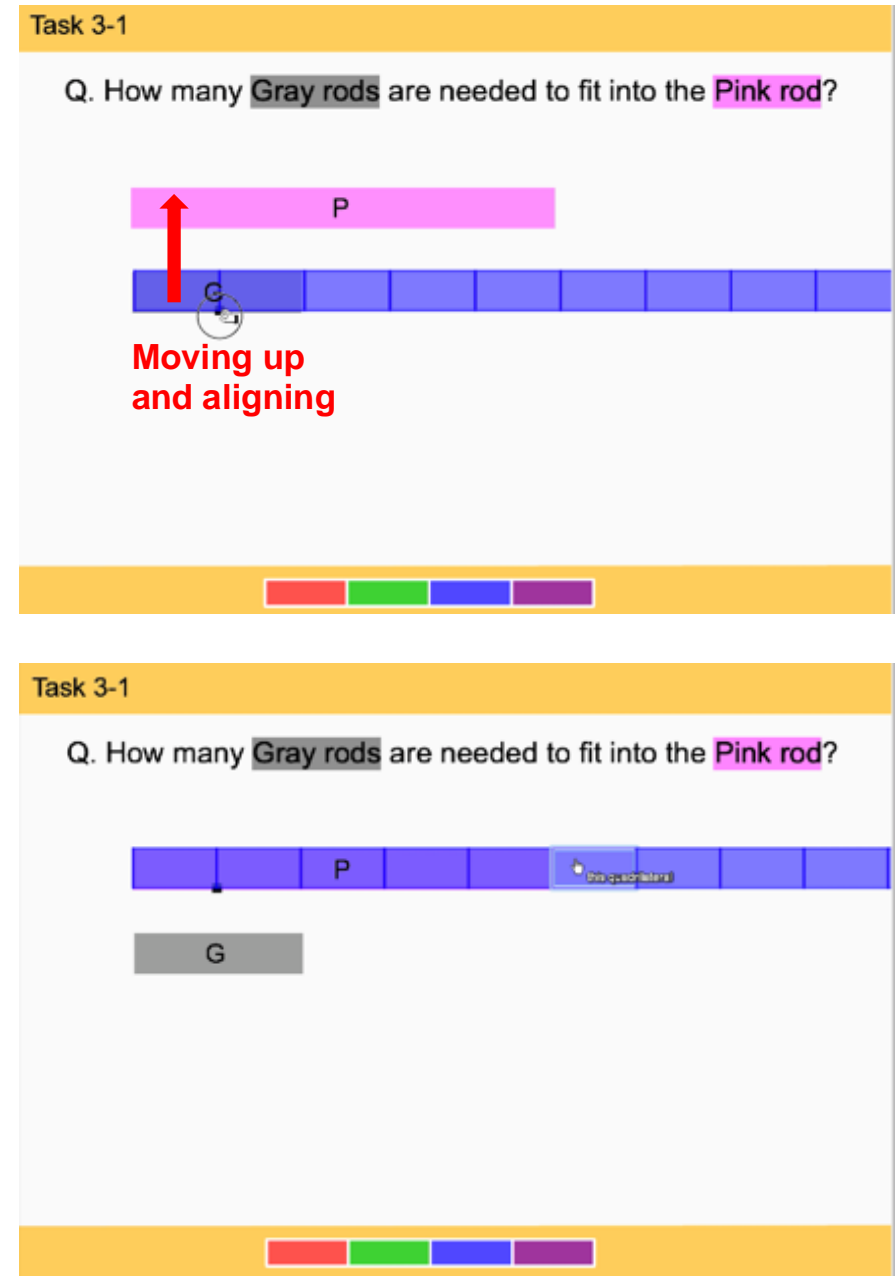

Note. In the top image of this figure, the gray rod overlapped by the Blue Dynamic Ruler, which is why the letter $\mathrm{G}$ appears in horizontal alignment with the ruler. In the bottom image, the pink rod is in alignment with the Blue Dynamic Ruler. 
Second, children could vary the size of the Dynamic Ruler's pieces by horizontally dragging a black dot that was a feature of the tool. If a child dragged the black dot left, the length of the pieces got smaller. If they dragged the dot to the right, the pieces got longer. For example, a child made the pieces on the Red Dynamic Ruler the same size as the yellow rod (Figure 24-top; in this image, the Red Dynamic Ruler is behind the yellow rod). The child made the Green Dynamic Ruler's pieces double the size of the yellow rod by dragging the ruler's black dot right (Figure 24-middle) and the Purple Dynamic Ruler's pieces half the size of the yellow rod by dragging its black dot left (Figure 24-bottom). I only counted as this dragging action when children had a purpose to quantify the length with an adjusted size of the Dynamic Rulers rather than just random manipulations.

\section{Figure 24}

Dragging: Changing Sizes

Task 1-1

Q. How many Yellow rods are needed to fit into the Blue rod?

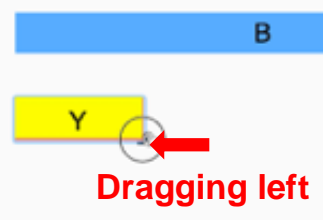


Q. How many Yellow rods are needed to fit into the Blue rod?

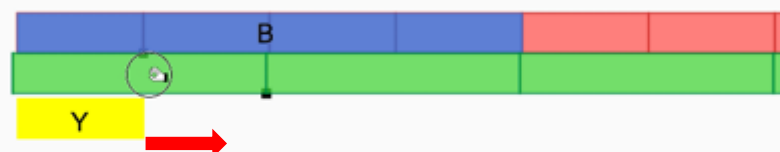

Dragging right

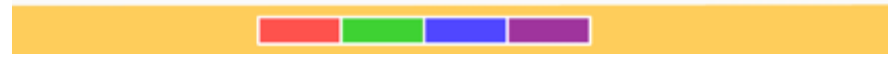

Task 1-1

Q. How many Yellow rods are needed to fit into the Blue rod?

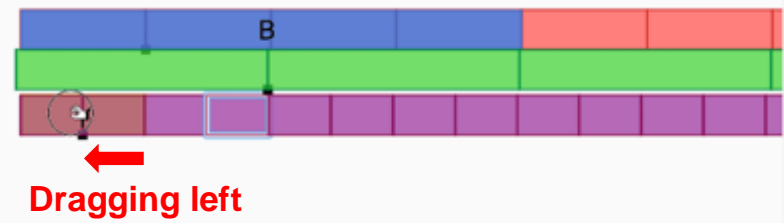

Dragging left

Note. In the top image of the figure, the Red Dynamic Ruler is only one piece long and behind the yellow rod. In the middle image, the blue rod overlapped by the Red Dynamic Ruler, which is why the letter B appears in horizontal alignment with the ruler. In the bottom image, the blue rod and Red Dynamic Ruler remain in alignment. Also, the yellow rod is in alignment with the Purple Dynamic Ruler, which is why the letter Y appears in horizontal alignment with the ruler (somewhat obscured by the tool's cursor in the leftmost unit of the Purple Dynamic Ruler).

Third, some children manipulated a Dynamic Ruler's pieces to find out what specific size was best by commeasuring both rods at the same time. This commeasuring 
action with the Dynamic Ruler was not a single action but a series of continuous actions: changing the size and checking the alignment of the Dynamic Ruler with two rods. For example, when a fifth grader was asked to justify their answer in Task 3-2 (Figure 25), she articulated how she arrived at a fractional expression through a commeasuring action: "I tried to make this blue block [fit] to one gray rod, but it can't fit into the pink rod. And I have tried to make gray rods a half, but it can't fit. So, I make more smaller than the half, and it can't fit. So, more, tinier. I can make four of them. It can fit. The four of blue rods are one whole gray rod. So, four is one whole. One, two - one, two, three, four [indicating that four of the Blue Dynamic Ruler's pieces fit into the gray rod]. Two and three fourths [indicating that $23 / 4$ gray units fit into the pink rod]."

Figure 25

Dragging: Commeasuring

Task 3-2

Q. How many Gray rods are needed to fit into the Pink rod?

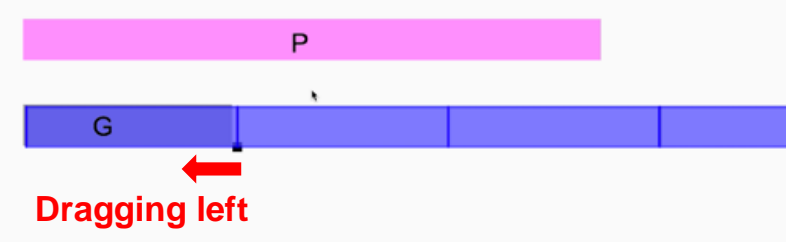




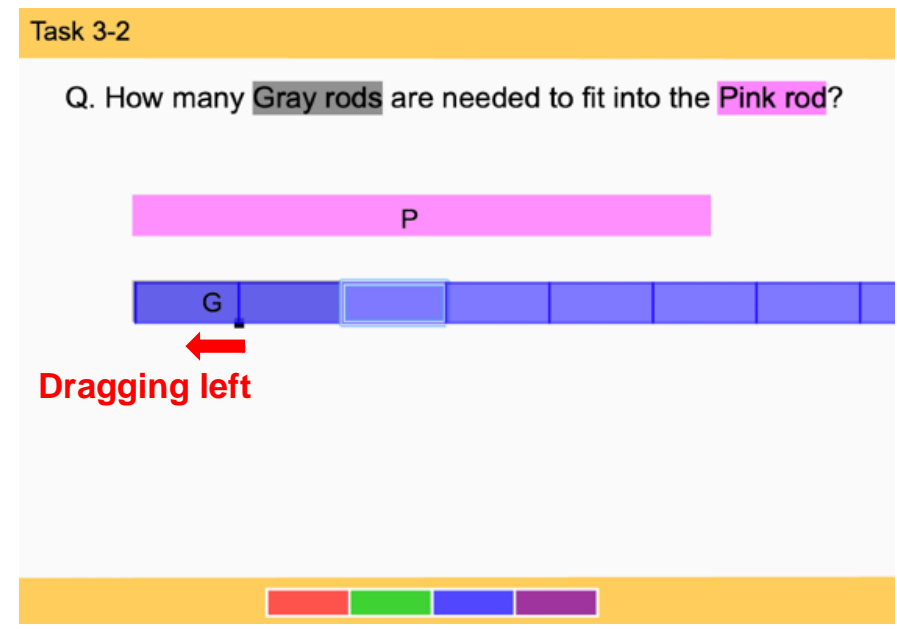

Task 3-2

Q. How many Gray rods are needed to fit into the Pink rod?
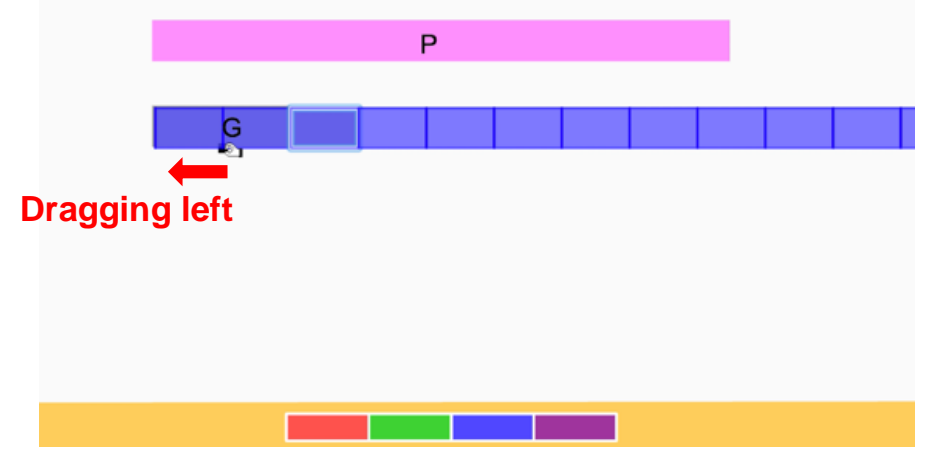

Task 3-2

Q. How many Gray rods are needed to fit into the Pink rod?
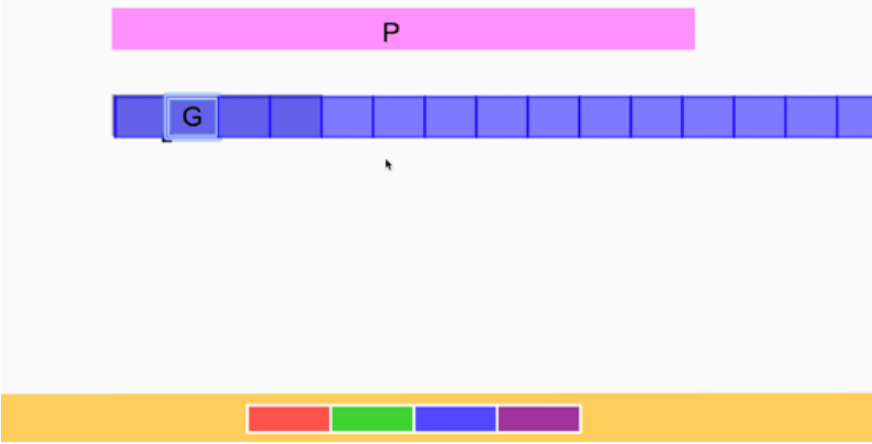

Note. The gray rod overlapped by the Blue Dynamic Ruler, which is why the letter G appears in horizontal alignment with the ruler. 
Mathematical ideas consisted of unit, fractions, and fraction as measure. Each subcategory had several codes. The unit subcategory contained three codes: unitizing, unit relation, and partitioning. First, when the children participating in the study verbalized their answers for the tasks, they had to decide which size was the unit of measurement. (For example, in the interviews, one student said, "So, if I move this or use this, I make something that's half the size." Episodes of the verbatim interviews are reported in Chapter 4.) There were various types of unitizing: whole size, double size, partial size, and random size. Second, children articulated unit relations (e.g., "If you use your purple, it takes two purple to make up the yellow."). There were two types of relationships between units: the given unit to a new unit and a new unit to the given unit. To solve the tasks, the students had to distinguish between the two types by identifying which part was the whole unit that served as the referent unit. In the former case, the whole was the new unit size, whereas, in the latter case, the given unit was the whole size need to compare the lengths of the two rods. Within the unit subcategory, the third code was partitioning, which refers to physically or mentally slicing an object into same-sized units to facilitate length measurement (e.g., "So, one whole nothing lines up with that. Half. When you go to half, you can see that that line right there, the end of the one, two, three - the fifth section lines up with the end of the pink rod.").

The second subcategory in the coding scheme's mathematical ideas category was fractions. This subcategory involved fractional notations, the concepts of equivalence and equivalent fractions, and arithmetic operation with fractions. First, the children expressed a partial amount with fractional notations (e.g., "One and one fourth orange rods are needed to fit into the brown rod"). They used multiple types of fractions, such as proper 
fractions, improper fractions, and mixed numbers (even sometimes decimals). Second, children described the equivalence between two values (e.g., "I mean four of the green sections fit into the brown, which is, um, four thirds. Um, and if you change that to a mixed number, one and one third.") and even equivalent fractions (e.g., "That means they're two eighths more filling that, and that's equal to one fourth more.”). Third, children demonstrated their understanding of arithmetic operations with fractions, such as multiplication and addition (e.g., "One section of the red is equal to one half of the orange section. So, one half. It goes one half plus one half. You do that three times: one half plus one half and one half. That's your equation. So that gets to one and a half.").

The children's conceptions of fraction as measure included how they used and thought about unit fractions and how they coordinated units to measure quantities. First, the children recognized a fractional part as a unit fraction. They often understood the relationship between whole units and unit fractions (e.g., "So, let's say we do half. And you know it's half because there are two sections, and each one of them is one of the two sections. Um, and that one is half of two"). Second, the units might have multiple layers as a composite unit, which refers to a unit containing another unit. The children explained how a partial unit coordinates with a whole unit to measure the whole unit's size and, subsequently, how the composite unit could measure the quantity (e.g., "So that means that there would be eight sections, and since three blue sections are equal to one gray section, there will be one that would be - this would be one gray, two grays - two grays and then two thirds of a gray. So, two and two thirds.").

In the second phase of coding, I used axial coding to identify the relationship between mathematical ideas and mathematical actions. This axial coding related 
categories to subcategories and showed the links between them as I learned about the study subjects' experiences to represent the categories (Charmaz, 2014, p. 61). The distinct categories (mathematical ideas mathematical actions described in Table 3), subcategories (unit, fractions, fraction as measure, hovering, dragging), and links reflected how I made sense of the data (Figure 26).

\section{Figure 26}

\section{Axial Coding}

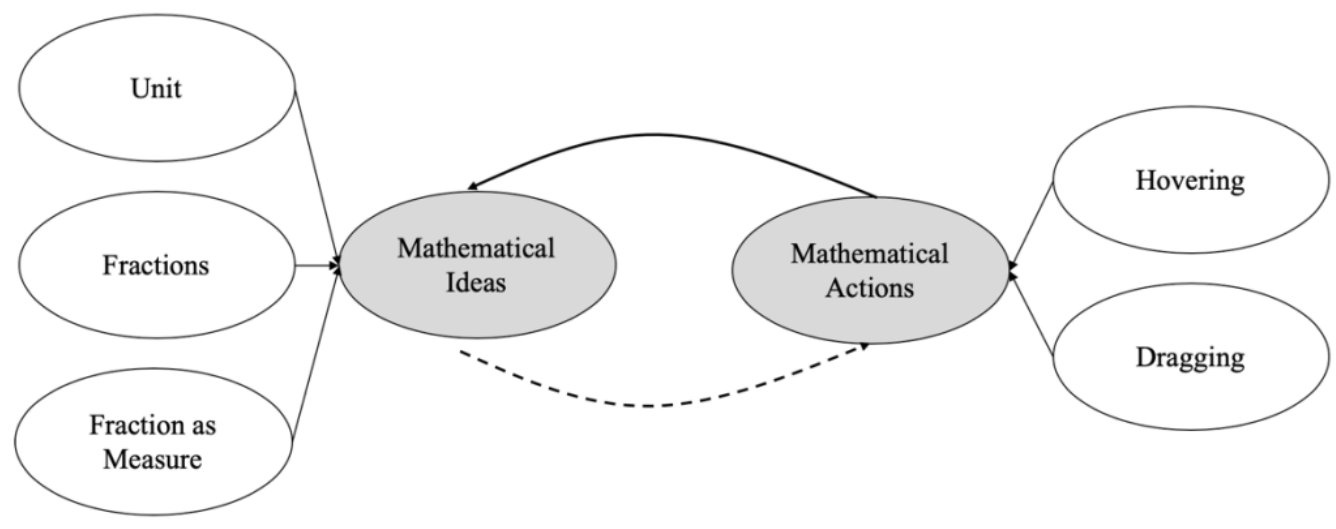

\section{Development of the Strategy Types Framework}

The coding scheme provided a tool for in-depth analysis of children's fraction understanding and use of technology. However, it was unrealistic to expect that posing the tasks would only elicit either mathematical thinking or mathematical action; the fraction-as-measurement tasks simultaneously involved fraction understanding as well as actions that required the use of technological features.

I developed a framework based on the axial coding to analyze the students' types of strategy concerning fraction as measure (Table 4). This framework also considered the context - the fact that the students' strategies interacted with the dynamic tool. The core 
idea central to solving the tasks was the relationship between the leftover piece and the original unit, which the children described by using the Dynamic Ruler. Commeasuring the given quantities with partial units, the students could interpret the result in terms of a fractional value with the given unit as the referent whole. As mentioned earlier in this chapter, I began developing the conceptual framework by running pilots. Based on two pilot studies, I developed the holistic analytic framework revealed by the pilot subjects' specific actions, operations, and verbal descriptions. The unit of analysis was a student's initial solutions in each fraction-as-measurement task.

As shown in Table 4, I classified solution strategies in terms of the extent of interaction between a child and a tool. If students are categorized in Type A of the framework, it means that the students might be careless about the relationship between the leftover and the unit size. Students also might not use the Dynamic Ruler at all to solve the given tasks. In Type B, students might visually notice the relationship between the leftover and the given unit and identify the result in terms of simple ratios, such as one half and one fourth. However, they do not explore the relationship with the Dynamic Ruler. In Type C, students might estimate the simple ratios (even the less obvious ratios, such as one third) with the Dynamic Ruler to test the relationship between the leftover and the original unit, but they do so inconsistently. In Type D, students begin to use the Dynamic Ruler consistently for situations where the ratio is $1 / n$ into a proper fraction $(\mathrm{m} / \mathrm{n})$. Students might move to more abstract types of strategy and operate three-level composite units (original unit, $1 / n$, and $n / m$ ). In Type E, students can use the Dynamic Ruler to commeasure both a given length and a unit without first making a copy of the leftover and placing it next to the original unit. 
During the actual analytic procedure, I refined and redefined the framework.

Table 4 focuses on the preliminary framework, but the refined framework will be introduced in the first part of Chapter 4.

\section{Table 4}

Preliminary Framework for Strategy Types of Fraction as Measure

\section{Type Students' strategies for solving the Fraction as Measure tasks}

Type A Gives little attention to relating the leftover to the original unit (e.g., "I need one orange rod")

Type B Estimates simple ratios (one half, one fourth) only

Type C Estimates simple and less obvious ratios (one third); may use the Dynamic Ruler inconsistently to check the relationship

Type D Consistently uses the Dynamic Ruler to determine the relationship between the leftover and the unit for $1 / n$

Type E Generalizes the use of the Dynamic Ruler by commeasuring both quantities

Furthermore, I identified how students approached different tasks as they worked with the tool and analyzed the changes of strategy types across multiple tasks. I used the strategy types framework to analyze the individual solution within and across Task Series 2 and 3 and focused on the changes in strategy types by examining consecutive tasks as the unit of analysis. With this approach, I could also identify qualitative changes by observing each case and documenting critical interactions. For the documentation, I employed analytic memos, each of which was "a brief or extended narrative that documents the researcher's reflections and thinking processes about the data" (Miles et al., 2013, p. 97). I documented the most salient features in each task and reviewed pairs 
of memos to identify what strategies students' employed and how they changed their strategies between tasks (Figure 27). I also used color-coding for specific memos to find representative cases to represent important variations (blue), typical cases to represent a common example (green), and special cases to represent interesting, unusual, or special attributes (yellow). For example, as a representative case, I highlighted the memo about Semi solving Task 1-2. Semi's initial strategy in Task 1-1 was to match the size of the given unit to the size of the Dynamic Ruler's pieces. However, after exploring various unit sizes, she used the halves as a unit to measure the given length in Task 1-2. She also had a clear understanding of the reverse relationship between the unit and the length with the same representations. When asked how much of a longer rod was needed to measure the shorter rod, she articulated the answer would be a third of a blue rod ("reverse problem: $1 / 3$ of blue rod" noted in her analytic memo in Figure 27).

Figure 27

Analytic Memos

\begin{tabular}{|c|c|c|c|c|c|c|}
\hline$\nabla$ & $1-1$ & $\nabla$ & $1-1$ & $1-2$ & $\nabla$ & $1-2$ \\
\hline Sewoong & $\begin{array}{l}-4 \text { (measuring yellow and count the number of reds) } \\
\text { - two of yellow, half of yellow (two times two, two } \\
\text { time two) } \\
\text { - "half of blue have four quarters" }\end{array}$ & $\begin{array}{l}\text { C/unit } \\
\text { iteration }\end{array}$ & 1 to 2 & $\begin{array}{l}-3 \text { with iteration }=6 \text { havles } \\
\text { - original/double/half - change the way to count }\end{array}$ & $\begin{array}{l}\text { C/unit } \\
\text { iteration }\end{array}$ & 1 to 2 \\
\hline Semi & $\begin{array}{l}\text { - } 4 \text { (measuring yellow and count the number of reds) } \\
\text { - half of yellow (two greens make one whole yellow) } \\
\text { - double, original, half }\end{array}$ & $\begin{array}{l}\text { C/unit } \\
\text { iteration }\end{array}$ & 1 to 2 & $\begin{array}{l}-3 \text { with iteration of halves } \\
\text { - reverse problem: } 1 / 3 \text { of blue rod }\end{array}$ & $\begin{array}{l}\mathrm{C} / \text { unit } \\
\text { iteration }\end{array}$ & 2 \\
\hline Sandol & $\begin{array}{l}\text { - four (using the given size at first but measuring } \\
\text { yellow) } \\
\text {-bigges rods make smaller number }\end{array}$ & $\begin{array}{l}\text { C/unit } \\
\text { iteration }\end{array}$ & 0 to 1,2 & $\begin{array}{l}\text { - } 3 \text { with iteration of whole yellow } \\
\text { - reverse problem: three rods (did not get this) }\end{array}$ & $\begin{array}{l}\text { C/unit } \\
\text { iteration }\end{array}$ & 1 \\
\hline Sungho & $\begin{array}{l}\text { - four (smaller rectangles, centimeter = unitizing) } \\
\text { - one mutiple four is four (multiplicative thinking) }\end{array}$ & $\begin{array}{l}\text { C/unit } \\
\text { iteration }\end{array}$ & maybe 2 & $\begin{array}{l}\text { - } 3 \text { with iteration of whole yellow (challenging } \\
\text { interpretation with halves) } \\
-9 \text { blues ( } 1 \text { blue }=3 \text { centi, }(3 \text { millimeter }) \text { ) }\end{array}$ & $\begin{array}{l}\text { C/unit } \\
\text { iteration }\end{array}$ & 1 \\
\hline Peter & $\begin{array}{l}\text { - four with iteration unit (separate DRs) } \\
\text { - one green piece, two green pieces: different units } \\
\text { - take more space makes smaller }\end{array}$ & $\begin{array}{l}\text { C/unit } \\
\text { iteration }\end{array}$ & 1 to 2 & $\begin{array}{l}\text { - about } 3 \text { with iteration whole yellow } \\
\text { - half is under the letter }\end{array}$ & $\begin{array}{l}\text { C/unit } \\
\text { iteration }\end{array}$ & $\begin{array}{l}1 \text { to not } \\
2\end{array}$ \\
\hline
\end{tabular}


I looked for evidence of how students' mathematical thinking changed as they used the Dynamic Rulers in successive scenarios. For that, my analysis focused on identifying and refining the mediated conceptions that children expressed across the tasks. I had particular questions to focus the analysis, such as, "How does a technological tool differently mediate children's understanding of fractions within the Dynamic Ruler environment?" I compared various patterns to identify themes in how the children engaged with the tasks and how they reasoned about unit and measure with the dynamic tool. Through this comparison, three themes emerged: (1) Different types of tasks impact different types of strategy; (2) Technological features have reciprocal relationships with mathematical understanding; (3) Mathematical actions with a tool mediate the way participants conceptualize mathematical meaning. This analysis illuminated new aspects of the measurement approach to fractions and has considerable implications for how mathematical thinking is mediated by the use of technology. 


\section{CHAPTER 4: FINDINGS}

As a reminder, the two research questions of the study are as follows: What are the different ways that children use the Dynamic Ruler tool in approaching each fractionas-measurement task? What patterns emerge in children's strategies across the series of the fraction-as-measurement tasks?

To answer the first research question, I developed a framework based on strategy types indicated by children's solutions to a series of tasks within the Dynamic Ruler environment and stemmed from the mathematical actions in manipulating the tool. The framework was focused primarily on how children unitized the segments of the Dynamic Rulers and how they coordinated those units to measure linear quantities. Each strategy type consisted of distinctive features of the children's interaction with the Dynamic Ruler and was accompanied by detailed descriptions of the actions and descriptions of participants involved in the tasks.

In this chapter, I first present the framework used to investigate how children engaged in different types of strategy in fraction-as-measurement tasks. After providing an overview of the framework, I present a detailed analysis of each type. Afterward, I present case analyses of how different types of strategy emerged from different tasks.

\section{Students' Strategy Types Framework on Fraction as Measure}

\section{Developing a Framework for Students' Strategies}

Based on two pilot studies, children's strategies to solve the tasks were categorized by their strategy types. Determined by specific actions, operations, and verbal descriptions, a strategy type framework of fraction as measure emerged from the analysis of Task Series 2 and 3 (Table 5). As described in Chapter 3, in Task Series 2 and 3, a 
quantity could not be precisely measured by the given unit length due to a leftover piece of intermediate length between the two rods' lengths in contrast to Task Series 1 . This leftover piece was the major component children were asked to interpret in the tasks. There were four types of strategy about unit relation that emerged, which I described as not attending to the relationship, estimating the relationship, determining the relationship, and commeasuring to find the relationship. Even though children used similar types of strategy, the way they dealt with the units varied based on their understanding of fractions, length measurement, and technological features. Note that I used this strategy type framework to analyze their initial solution strategy in solving each task as the unit of analysis in order to capture their own strategies without the interviewer's probing and questioning. I will address the effect of such questioning in the findings for Research Question 2.

\section{Table 5}

Types of Children's Strategies for Fraction as Measure Tasks

\begin{tabular}{ll}
\hline \multicolumn{1}{c}{ Types } & \multicolumn{1}{c}{ Description } \\
\hline Est Attending & Pays little attention to the leftover part \\
& $\begin{array}{l}\text { Pays attention to the relationship of (or even measurement of) the } \\
\text { leftover part but still estimates using the given original unit size }\end{array}$ \\
Determining & $\begin{array}{l}\text { Determines the relationship between the given unit and } \\
\text { intermediate units using Dynamic Rulers }\end{array}$ \\
Commeasuring & $\begin{array}{l}\text { Measures the given unit and quantity subsequent to (or } \\
\text { simultaneously with) the scaled partial unit sizes of Dynamic } \\
\text { Rulers }\end{array}$ \\
\hline
\end{tabular}


In the following sections, I describe each strategy type in detail, providing examples of how children manipulated Dynamic Rulers to coordinate unit lengths within each task.

\section{Fraction as Measure Using the Dynamic Ruler}

Table 6 summarizes the different types of strategy evidenced in Task Series 2 and 3. In the category type labeled Not Attending, although students might have adjusted the unit size of the Dynamic Ruler relative to the given unit, students paid little attention to the leftover amount and counted only the whole number of units. In the type designated Estimating, children took visual notice of the relationship between the leftover length and the given unit and then identified the result using simple ratios, such as one half and one fourth. However, they chose not to explore the relationship using Dynamic Rulers between the original unit and the leftover length. In the type designated Determining, students measured the whole-number parts using pieces of a Dynamic Ruler that were the same size as the given unit. Then they generated a new intermediate unit to measure the leftover. In the type called Commeasuring, students began to use a scaled size unit compared to the given unit. For example, some students first measured the given unit with a newly generated partial unit and then figured out the size of the partial unit in the quantity to be measured. Others partitioned the given unit and the quantity simultaneously by finding a unit of measure on the Dynamic Ruler that was able to measure both. These students at this type could use the Dynamic Ruler to commeasure both a quantity and a unit length without having to make a copy of the leftover and place it alongside the original unit. Some children even anticipated various equivalent fractions based on multiplicative reasoning. 
Table 6

Types of Students' Strategies About Fraction as Measure

\begin{tabular}{|c|c|c|}
\hline Type & \multicolumn{2}{|r|}{ Description and example } \\
\hline \multirow[t]{6}{*}{ Not Attending } & \multicolumn{2}{|c|}{ Pays little attention to the leftover length and measure unit } \\
\hline & \multirow{5}{*}{$\begin{array}{l}\text { Child measures } \\
\text { the quantity (P) } \\
\text { with a ruler piece } \\
\text { the same size as } \\
\text { the given unit (G) } \\
\text { but does not } \\
\text { recognize the } \\
\text { leftover part as a } \\
\text { number (e.g., } \\
\text { "two blues"). }\end{array}$} & Task 3-1 \\
\hline & & \multirow[t]{2}{*}{ Q. How many Gray rods are needed to fit into the Pink rod? } \\
\hline & & \\
\hline & & G \\
\hline & & 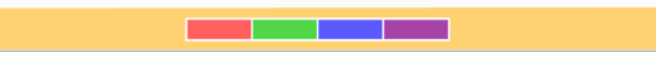 \\
\hline \multirow[t]{6}{*}{ Estimating } & \multicolumn{2}{|c|}{$\begin{array}{l}\text { Notes the relationship (or even measures) the leftover length } \\
\text { but still estimates using the given original unit size }\end{array}$} \\
\hline & \multirow{5}{*}{$\begin{array}{l}\text { Child notes the } \\
\text { leftover related to } \\
\text { the given unit (G) } \\
\text { by estimating } \\
\text { how much the } \\
\text { leftover is or how } \\
\text { much more it } \\
\text { needs to be a } \\
\text { whole unit (e.g., } \\
\text { "two and a half," } \\
\text { "need a half for } \\
\text { 3"). }\end{array}$} & Task 3-1 \\
\hline & & \multirow[t]{2}{*}{ Q. How many Gray rods are needed to fit into the Pink rod? } \\
\hline & & \\
\hline & & G \\
\hline & & \begin{tabular}{|l|l|l|l} 
& & \\
\end{tabular} \\
\hline
\end{tabular}




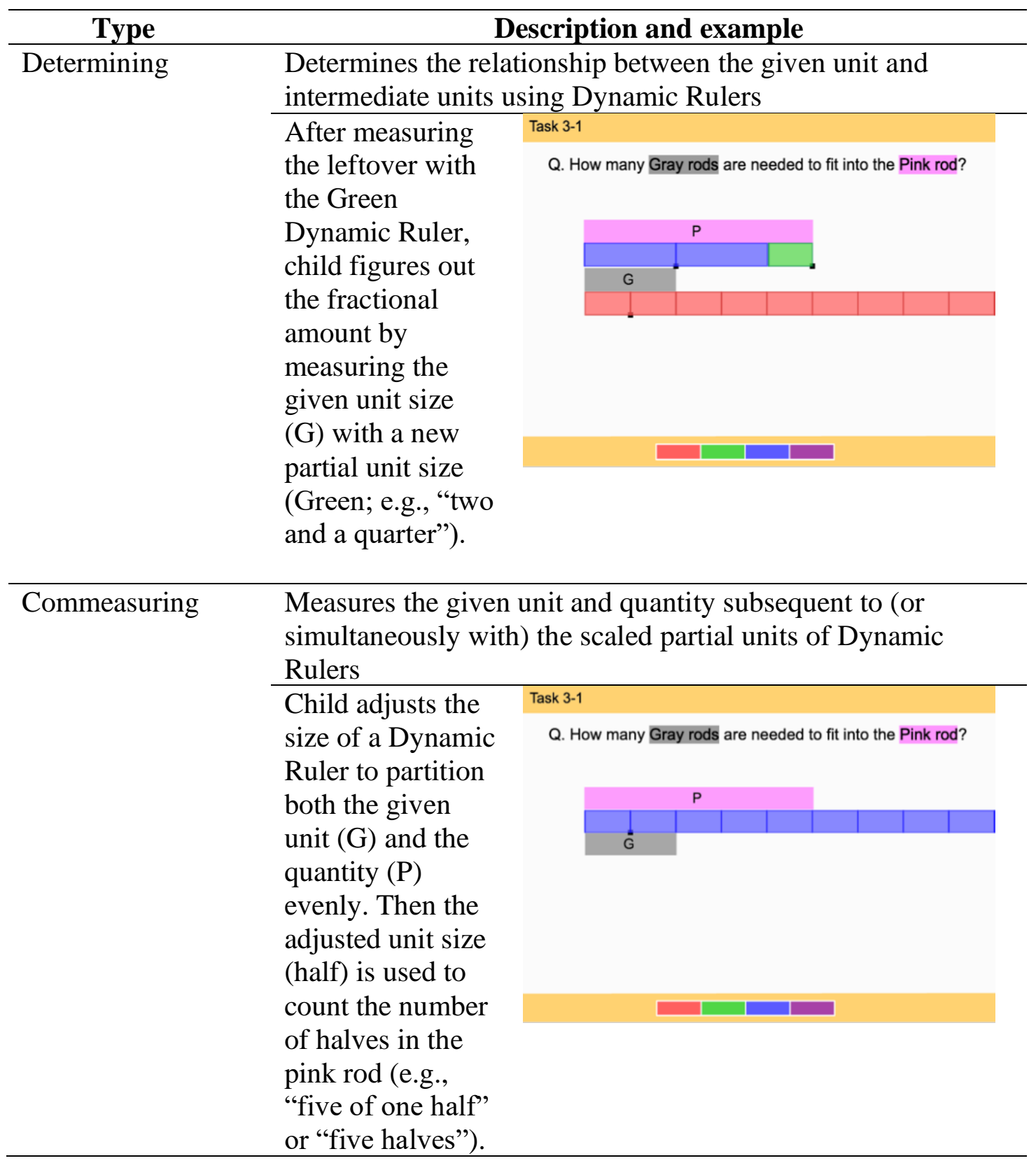

Students' strategies to solve fraction-as-measurement tasks were analyzed and categorized into the four types of strategy framework (Table 7). I did not include the third task in each series, which included prepartitioned rulers because there was not an opportunity for children to explore unit relationships by manipulating the Dynamic Rulers. 
Table 7

Distribution of Strategy Types by Tasks and Grade Levels

\begin{tabular}{|c|c|c|c|c|c|}
\hline Grade & Task & Not Attending & Estimating & Determining & Commeasuring \\
\hline Grade 3 & $2-1$ & 4 & 7 & 0 & 2 \\
\hline \multirow[t]{5}{*}{$(N=13)$} & $2-2$ & 3 & 5 & 0 & 4 \\
\hline & $2-4$ & 1 & 4 & 1 & 3 \\
\hline & $3-1$ & 0 & 9 & 2 & 2 \\
\hline & $3-2$ & 2 & 4 & 0 & 6 \\
\hline & $3-4$ & 1 & 2 & 0 & 4 \\
\hline Subtotal & & 11 & 31 & 3 & 21 \\
\hline Grade 4 & $2-1$ & 1 & 5 & 0 & 2 \\
\hline \multirow[t]{5}{*}{$(N=8)$} & $2-2$ & 1 & 1 & 2 & 4 \\
\hline & $2-4$ & 0 & 1 & 2 & 4 \\
\hline & $3-1$ & 0 & 3 & 1 & 4 \\
\hline & $3-2$ & 1 & 1 & 3 & 3 \\
\hline & $3-4$ & 0 & 1 & 3 & 3 \\
\hline Subtotal & & 3 & 12 & 11 & 20 \\
\hline Grade 5 & $2-1$ & 0 & 3 & 2 & 4 \\
\hline \multirow[t]{5}{*}{$(N=9)$} & $2-2$ & 0 & 1 & 2 & 6 \\
\hline & $2-4$ & 0 & 0 & 2 & 6 \\
\hline & $3-1$ & 0 & 1 & 2 & 6 \\
\hline & $3-2$ & 0 & 1 & 2 & 6 \\
\hline & $3-4$ & 0 & 0 & 2 & 6 \\
\hline Subtotal & & 0 & 6 & 12 & 34 \\
\hline Total & & 14 & 49 & 26 & 75 \\
\hline
\end{tabular}

Table 7 summarizes the distribution of strategy types in Task Series 2 and 3. The Not Attending Type appeared dominantly with third graders, and the frequency was lower than at other types overall. In fifth grade, this type of strategy was not present. Most of students began to gain some familiarity with how to manipulate the Dynamic Ruler tool 
as they proceeded through the series of tasks, though one or two students continued to struggle with measurement concepts (such as unitizing and unit coordination). The strategy type of those in the Estimating Type was the most straightforward, with 49 examples among the student respondents. To be specific, third-grade children frequently used this type of strategy by matching the Dynamic Ruler pieces to the given length. When they solved the simple ratio fraction (e.g., one half, one fourth), students could determine the leftover amount based on estimation. There were fewer examples of third and fifth graders included in the Determining Type than fourth graders. Fourth-grade students utilized additional Dynamic Rulers to measure the leftover with a new partial unit size. The Commeasuring Type was the most common strategy (75 examples) used to solve fraction-as-measurement tasks, and the percentage of those at this type increased as the grade levels went up. Among the instances noted, three fifth-grade students demonstrated a further potential use of the Dynamic Ruler tool when they generalized mathematical invariant properties, such as equivalence and the relation between unit size and measure.

\section{Illustration of Strategy Types for Fraction as Measure Tasks}

In this section, I describe examples of each type of strategy as determined by six tasks (2-1, 2-2, 2-4, 3-1, 3-2, and 3-4).

\section{Not Attending Unit Relation}

Some students only used the default size of the Dynamic Ruler to measure a quantity. The default size matched neither the given unit nor the quantity to be measured. When designing the Dynamic Ruler tool, I intentionally established this feature so that students might be motivated to adjust the size of the Dynamic Ruler, but some students in 
the Not Attending Type did not feel a need to change the size of the units or otherwise failed to characterize the relationship between the leftover and the original unit size. In the following section, I include two students' exemplified strategies: Mary and Sungho. Mary used the default size of the Dynamic Ruler to measure the top rod without adjustment of the size of the ruler, and Sungho matched the given unit size by adjustment of the tool but did not pay attention to a referent unit.

In Task 2-1, Mary used a strategy that used the default size of the Dynamic Rulers. Lining up the Green Dynamic Ruler between two rods with the dragging action, she counted the number of green pieces to determine the difference between the orange rod and the brown rod (Figure 28). She only paid attention to adjacent parts and stated: “One, two. Just two." However, the two pieces she indicated were of different lengths. The length of the first piece was the difference between the second green piece and the right end of the orange rod, while the length of the second piece was the difference between the right end of the brown rod and the two green pieces. (See the red rectangles in Figure 28-top.) Later, I asked her to make the orange rod the same size. The purpose of this directive was to prompt her to take the leftover into account, which was a fractional amount. However, her understanding of length measurement did not change, and she did not attempt to count the leftover using a partial unit. Her concluding answer to the number of pieces was “One.” (See Figure 28-bottom.) 


\section{Figure 28}

Mary's Use of the Default Size Unit

Task 2-1

Q. How many Orange rods are needed to fit into the Brown rod?
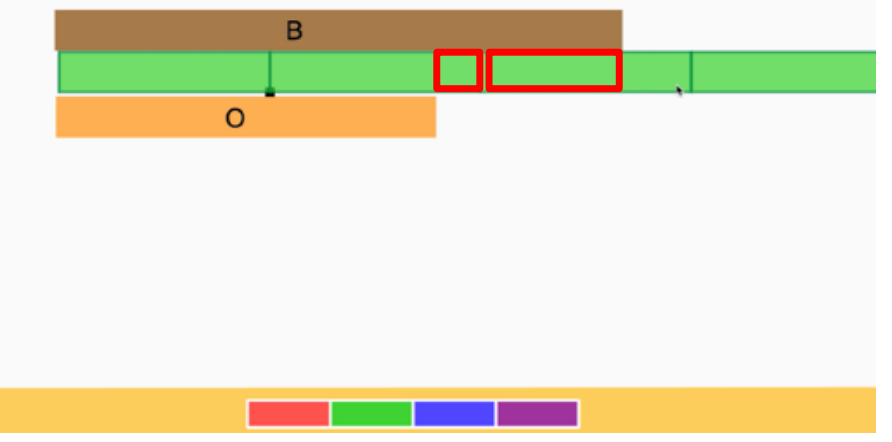

Task 2-1

Q. How many Orange rods are needed to fit into the Brown rod?
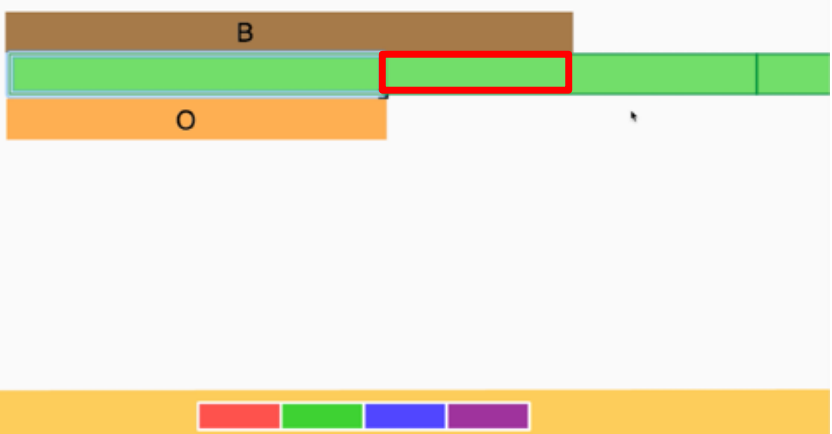

Mary was struggled to identify appropriate unit sizes to measure in the task (unitizing). She used only a default size of Dynamic Rulers in her initial approach to Tasks 2-1, and she counted the number of pieces in a pattern similar to that used in previous tasks. She needed two more green pieces, but the two pieces were of different sizes. The first piece was much shorter than the second one. This difference meant that her understanding of unit size was not fully established, and she perceived measuring as 
object-counting. She merely counted the number of pieces in the Dynamic Rulers without regard for their sizes.

When children solved Task Series 2 and 3, most of them began to measure the same size of a unit length by matching it to the Dynamic Ruler. However, some only considered the number of parts of the Dynamic Rulers, paying little attention to the relationship with the given unit. When Sungho, a fourth grader, was solving Task 2-1, he showed his strategy with the use of a partial unit through the dragging actions (i.e., lining up, changing size). After adjusting the size of the Green Dynamic Ruler (Figure 29), his initial response was three, "This rod B is three." Without making any relation to the orange rod, he just counted the number of green pieces. When pushed to use the orange rod to get the solution of the original prompt, he did not pay attention to the leftover and concluded that the answer was one orange rod.

\section{Figure 29}

Sungho's Estimation of the Length With a Partial Unit

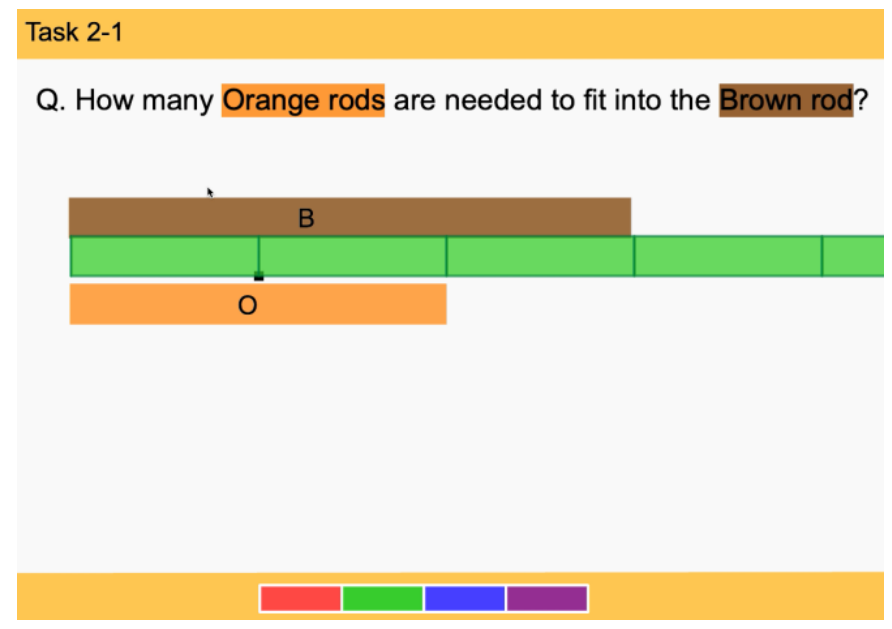


Even though children like Sungho understood measuring as unit-counting, they might have little foundation for understanding that the referent unit is significant to measure and the units must be exhausted in order to measure a quantity. He might have intuitively known that the length of the Dynamic Ruler could be varied by exploring size adjustment. However, he did not pay attention to the leftover amount and the unit relation.

In summary, in the Not Attending Type, there are two types of examples: 1) using the default size and counting the difference, ignoring partial pieces, and 2) making some adjustment to the Dynamic Ruler, maybe even matching the given unit, but still ignoring the partial pieces and answering with whole numbers.

\section{Estimating Unit Relation}

The most straightforward method of strategies that the children used to solve fraction-as-measurement tasks (Task Series 2 and 3) was to measure a given unit length through the dragging actions (e.g., lining up and changing size) and then to estimate the leftover as a fractional amount. When students measured a quantity with a unit length, they felt obliged to represent the leftover amount in relation to the original unit length. This approach was the main entry point by which the dynamic digital environment could introduce rational numbers in length measurement situations. In everyday experience, the terms "half" or "quarter" are well-known fraction expressions, and it is therefore expected that one would use these fraction words when comparing units and quantities. In the following section, I include four student examples from interviews with Lauren, Eric, Sumin, and Peter. 
Lauren, a third grader, matched the size of the Green Dynamic Ruler's pieces with the size of the orange rod in Task 2-1 (changing size). Then she moved the Dynamic Ruler up over the brown rod (lining up) and said, "Let's see. One and a half." When asked where the half was, she pointed to the leftover amount through the hovering action which indicated the middle of the second green piece with the cursor (Figure 30-top). She further explained the reason why the leftover was half of the unit length by making the same unit size with the Red Dynamic Ruler. She overlapped the red piece with the green piece (Figure 30-bottom), then pointed to the middle of the green piece and noted it was half of the original unit: "Cause estimating. I measure this big orange one because all of them lay out. Click there, and then you pull this one up here like that. And then this isthis is a half right there."

\section{Figure 30}

Lauren's Estimation of the Leftover

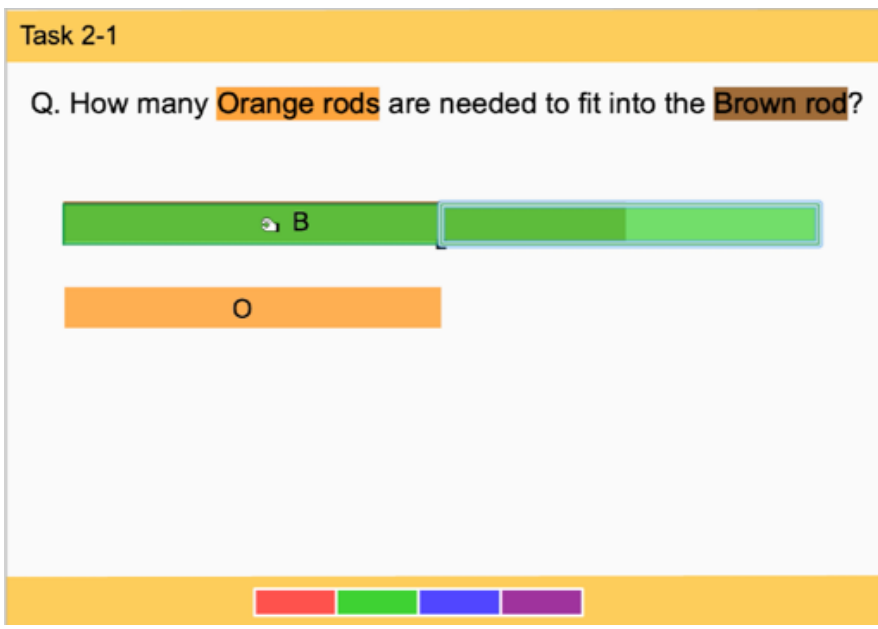




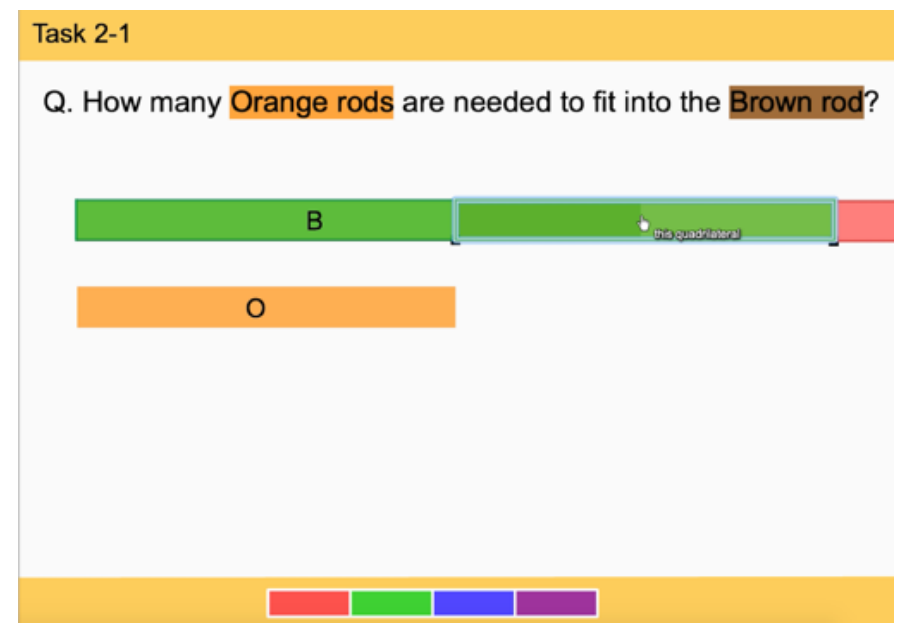

Note. The brown rod overlapped by the Green Dynamic Ruler, which is why the letter B appears in horizontal alignment with the ruler.

Eric, a fourth grader, used the same type of strategy to solve Task 3-1. He first matched one unit of the Blue Dynamic Ruler with the gray rod (lining up), making them the same size (changing size), and estimated the unit measurement size of the pink rod using a fraction term (Figure 31-top): "I'd say two and one half." When I asked where the half came from, he answered by resizing the Blue Dynamic Ruler into halves: "Because when I'd split the gray into a half, then I count one, two, three, four, five- Because if I'd measured the gray, I'd have two and then one left, and then that'd be a half." 


\section{Figure 31}

Eric's Estimation of the Leftover

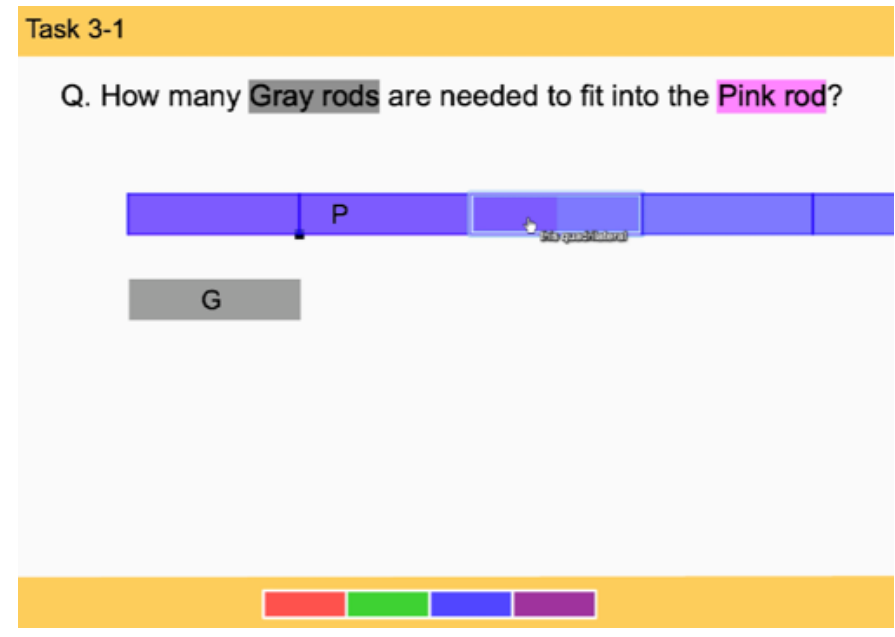

Task 3-1

Q. How many Gray rods are needed to fit into the Pink rod?
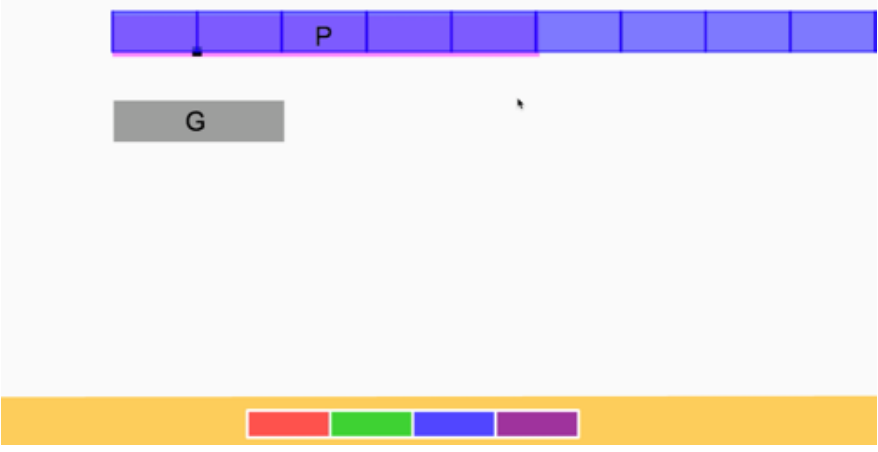

Note. The pink rod overlapped by the Blue Dynamic Ruler, which is why the letter $\mathrm{P}$ appears in horizontal alignment with the ruler.

In both cases, the children set out to measure the given unit length (unitizing), then compared the leftover amount in the other rod to the original unit size (unit relation). This strategy was not anticipated because the students usually paid more attention to matching the size of Dynamic Rulers with the unit length, and they thus easily coordinated the whole-number parts with the use of Dynamic Rulers. Still, they finished 
the tasks by estimating the leftovers. These estimations might have produced incorrect answers. This might be because some third-grade students do not learn all fraction terminology (such as one-third), and the Dynamic Ruler tool requires precision to adjust the sizes of units.

Children who conceived whole-number parts as obvious often used multiple Dynamic Rulers to figure out the relationship between the leftover part and the unit length. Students with this type of strategy used one Dynamic Ruler to represent the whole number (i.e., one or two), then used the dragging action by resizing a different colored Dynamic Ruler to measure the leftover part as intermediate units. In so doing, they could estimate the measured part in relation to the original unit size. Sumin, a fourth grader, measured the orange rod with the Green Dynamic Ruler in Task 2-1. She trimmed the number of pieces of the Dynamic Ruler and left only one of the green units under the brown rod (Figure 32-top). Sumin then decided to pull out the Blue Dynamic Ruler and measured the leftover amount with one blue piece (Figure 32-top). Her final answer to the problem was, "One orange and a half." To determine the size of a half, Sumin moved the blue piece over the orange rod (Figure 32-bottom) to compare sizes and estimated the size of the blue piece in relation to the orange rod. 


\section{Figure 32}

Sumin's Use of an Intermediate Unit

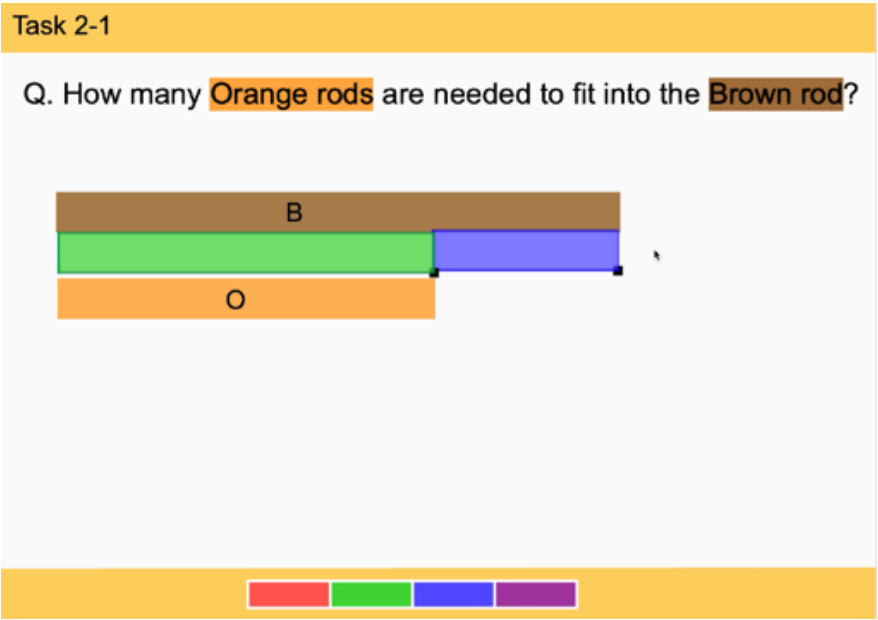

Task 2-1

Q. How many Orange rods are needed to fit into the Brown rod?
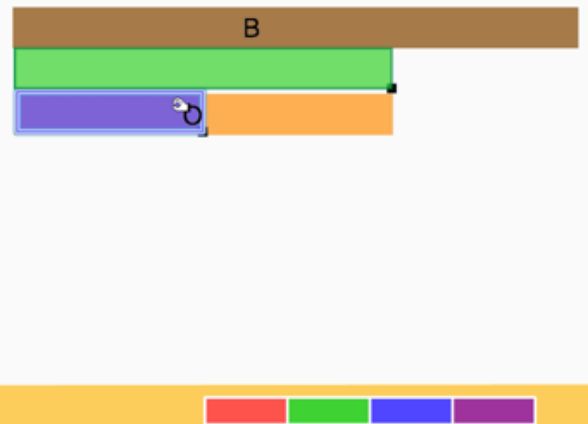

Peter, a third grader, at first matched the size of the Green Dynamic Ruler with the orange rod, then had one green piece left in Task 2-2 (Figure 33-top). He measured the leftover part with the Purple Dynamic Ruler and decided to trim the ruler to make only one purple piece. Peter manipulated the purple piece by making it half of the orange rod and shortening the size half again next to the green piece (Figure 33-middle). His first answer to the question was "one," but I pressed him to explain the meaning of the purple piece. He articulated the process of how he determined the size of the purple piece: "So, I 
took like half of it, like took half, put it right here [next to the green piece]. See that's about, like, half of half." He seemed to measure the leftover amount with the Purple Dynamic Ruler and ended up with a half-of-half-sized piece by estimating from the half size of the purple piece (Figure 33-bottom).

\section{Figure 33}

Peter's Use of an Intermediate Unit

Task 2-2

Q. How many Orange rods are needed to fit into the Brown rod?
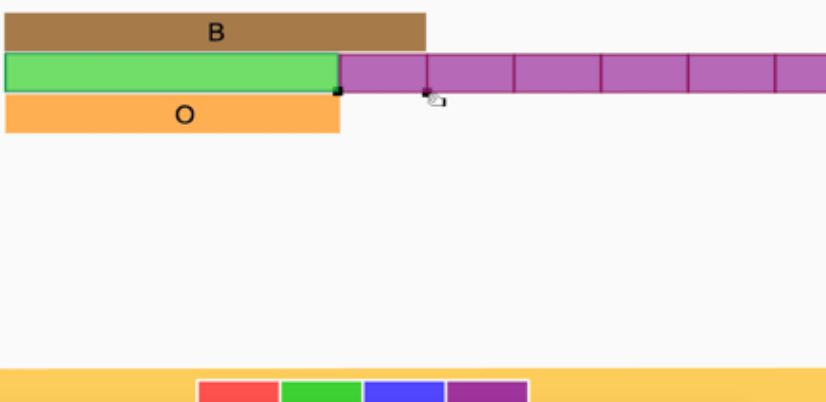

Task 2-2

Q. How many Orange rods are needed to fit into the Brown rod?

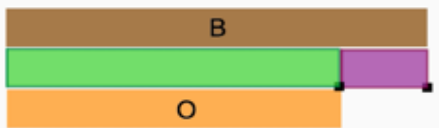




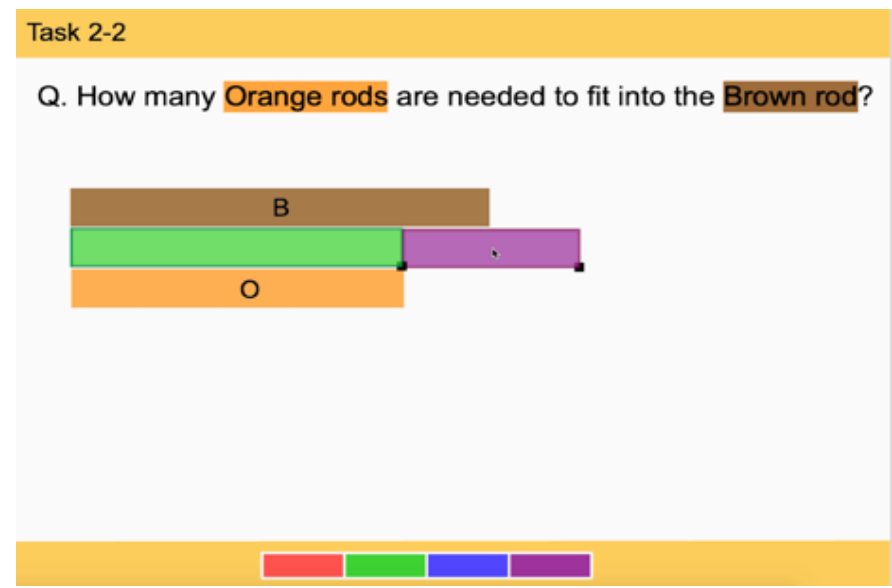

At this type, children could manipulate an additional Dynamic Ruler to measure the leftover part. They could not measure the size of the remainder using the original unit size. Therefore, this task provided crucial evidence of children's recognition of the leftover amounts as unique units. Even though some students used this intermediate unit size (Sumin and Peter), their ideas were as less straightforward as students who used the given unit size (Lauren and Eric). This similarity is because both groups of students estimated the leftover using fractional terms. However, using the intermediate unit might be preferable in the long term because it might allow students to develop a fundamental concept of fraction as measure through a dynamic direct comparison between the partial unit and the original unit.

\section{Determining Unit Relation}

Children carried out two processes in this type of strategy. First, they figured out the whole-number part by measuring a unit length (changing size of one ruler). Second, they measured the leftover with another smaller unit as an intermediate unit (changing size of the other ruler). They partitioned the unit length with partial units, then eventually, they have come to understand the relationship between the partial units and the given unit 
length. In the following section, I discuss two students, Charim and Sewoong. After measuring the whole-number part, Charim used multiple Dynamic Rulers to partition the given unit length with various sizes. Similarly, Sewoong used an additional Dynamic Ruler to verify the relationship between the given unit length and the leftover part.

Charim, a fifth-grade student, at first started to match the Green Dynamic Ruler's pieces to the orange rod in Task 2-2, then moved it up below the brown rod (Figure 34). She did the same manipulation with the Purple Dynamic Ruler and placed it next to the orange rod. She further partitioned the purple piece into halves with the Blue Dynamic Ruler and into fourths with the Red Dynamic Ruler. Finally, she answered: "One and one fourth," When asked why she said a fourth, she explained her way of measuring with fourths: "Because if it has two orange rods, it will be over the brown rod. And I so break the orange into a half, and I break the half into half. And it got me on the one fourth. If I add up one orange, which is one whole and one fourth, one and one fourth is the answer."

\section{Figure 34}

Charim's Use of an Intermediate Unit Task 2-2

Q. How many Orange rods are needed to fit into the Brown rod?

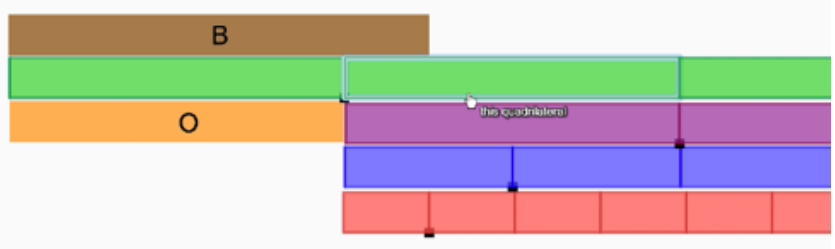


Sewoong, a third grader, initially manipulated the size of the Green Dynamic Ruler so that one unit on the ruler was the same size as the orange rod, as he did in previous tasks (2-1 and 2-2). Then, he pulled out the Red Dynamic Ruler to measure the leftover part (Figure 35). While he used additional Dynamic Rulers when I asked him to use those for probing, this was the first time he used multiple Dynamic Rulers of his own volition to figure out the leftover length more precisely. Sewoong used fourths to measure the leftover like in previous tasks, but it was not enough to measure the new leftover. Therefore, he decided to iterate the same strategy to measure the difference between the leftover and one red piece by using the Blue Dynamic Ruler (Figure 35). This type of an iterative measuring is clearly a distinctive action compared to Commeasuring type which generates new unit size to measure the leftover and the given unit simultaneously. He partitioned a fourth of the orange rod into four pieces and generated a unit on the Blue Dynamic Ruler that was $1 / 16$ the size of a unit on the Green Dynamic Ruler. Finally, his solution for the task was the sum of three different unit sizes (green: one whole, red: one fourth, blue: 1/16).

\section{Figure 35}

Sewoong's Use of Intermediate Units

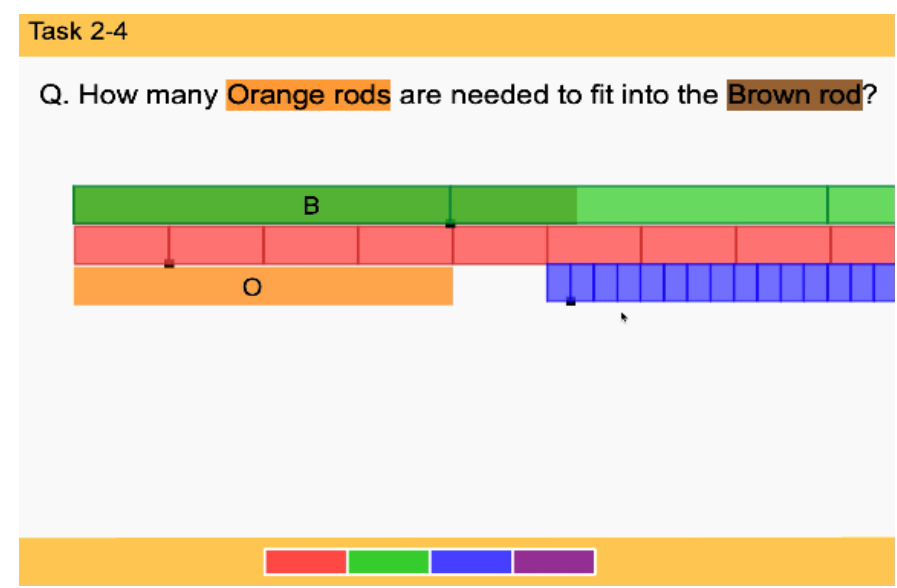

Note. The brown rod overlapped by the Green Dynamic Ruler, which is why the letter B appears in horizontal alignment with the ruler. 
Students in the Determining Type used recursive measurement to figure out whole numbers and fractional parts separately. The first measurement was to match the size of the unit length with one piece of the Dynamic Ruler. Subsequently, the leftover part was measured by new partial units, such as halves or fourths. This strategy required multiplicative thinking between two different unit sizes. The relation between units was proportional because children had to determine the partial unit size in relation to the original unit length. However, two different units were contained in the answer at the same time.

Unlike students in the prior strategy type (Estimating Type), these children did not use an estimate to figure out the leftover amount. Their use of fraction words derived from a second measurement process with partial units. This strategy could correspond to the next type of strategy that measures the quantity with only scaled (or partial) units.

\section{Commeasuring}

Children who have a strong idea of length measurement can use a single Dynamic Ruler to measure both a unit length and a quantity by partitioning them into similar partial units through resizing. They might already realize the unit length is not appropriate to measure the given quantity because there is a leftover when using the unit length. Then, they looked for a partial unit that could partition both rods evenly through the dragging action. Therefore, this type of strategy, which uses scaled units, can be viewed as anticipatory reasoning. The children in the Commeasuring Type had an intentional goal for their actions, which was to partition the unit length as well as the given quantity with common smaller units. In the following section, I include examples of four students: Teo, Arim, Gina, and Alex. Teo and Arim found one specific case of 
partial units to partition both rods. Gina and Alex found various equivalent fractions with the same value by continuously manipulating the Dynamic Rulers.

In Task 2-1, Teo, a fourth grader, gave an initial answer with an eyeballed approximation: "One and a half." He then explained how he arrived at that answer with the Green Dynamic Ruler (Figure 36): "If I take this off here, and I make it the same size [with two halves], it's, it's already going to be half of it. And then you take this you pulled up here. That's a full one and a half." When asked why he made a half size of the Green Dynamic Ruler, he said, "Well, why I decided to make this [is] because you can't make it bigger. You have to either stick with this or cut it into pieces or half." He also argued that only a smaller size could work to measure the unit.

\section{Figure 36}

Teo's Use of Partial Units

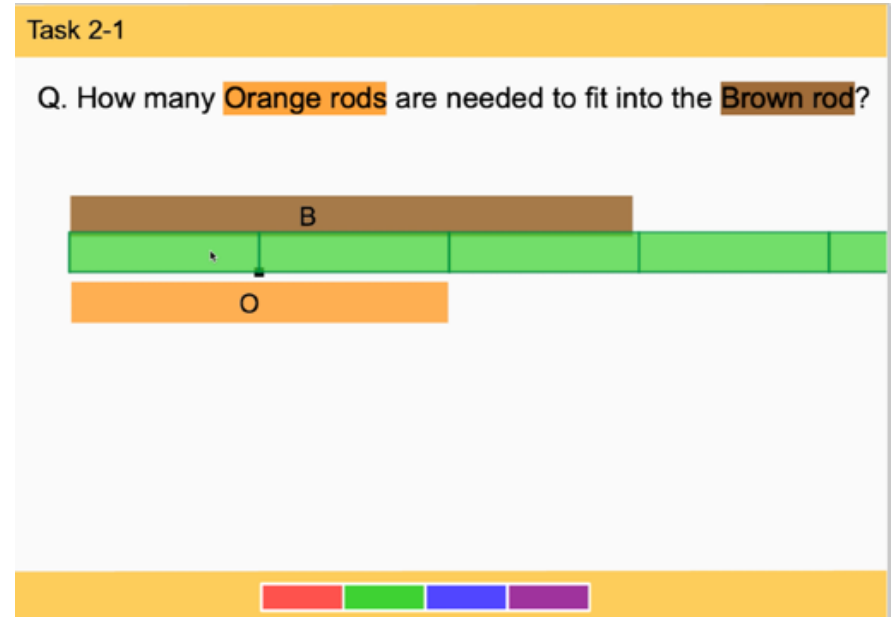

Arim, a fourth grader, manipulated the Blue Dynamic Ruler in Task 3-4 and, at first, matched it with the gray rod. She kept adjusting the size of the Dynamic Ruler, first as a half of the gray rod and then a third. Deciding to use thirds as a new partial unit, she 
marked groups of three blue pieces like rectangular shapes with the hovering action. She made the first rectangle with the three blue pieces (Figure 37-top) and marked the second one with six pieces (Figure 37-middle). The third group did not contain three pieces, so her final answer was "two and two third" (Figure 37-bottom). She also explained the relationships between both quantities and the Blue Dynamic Ruler: "[One blue piece unit] divides pink into eight pieces." She further explained that one gray rod is equivalent to "times three" of one blue piece.

\section{Figure 37}

Arim's Use of the Partial Units

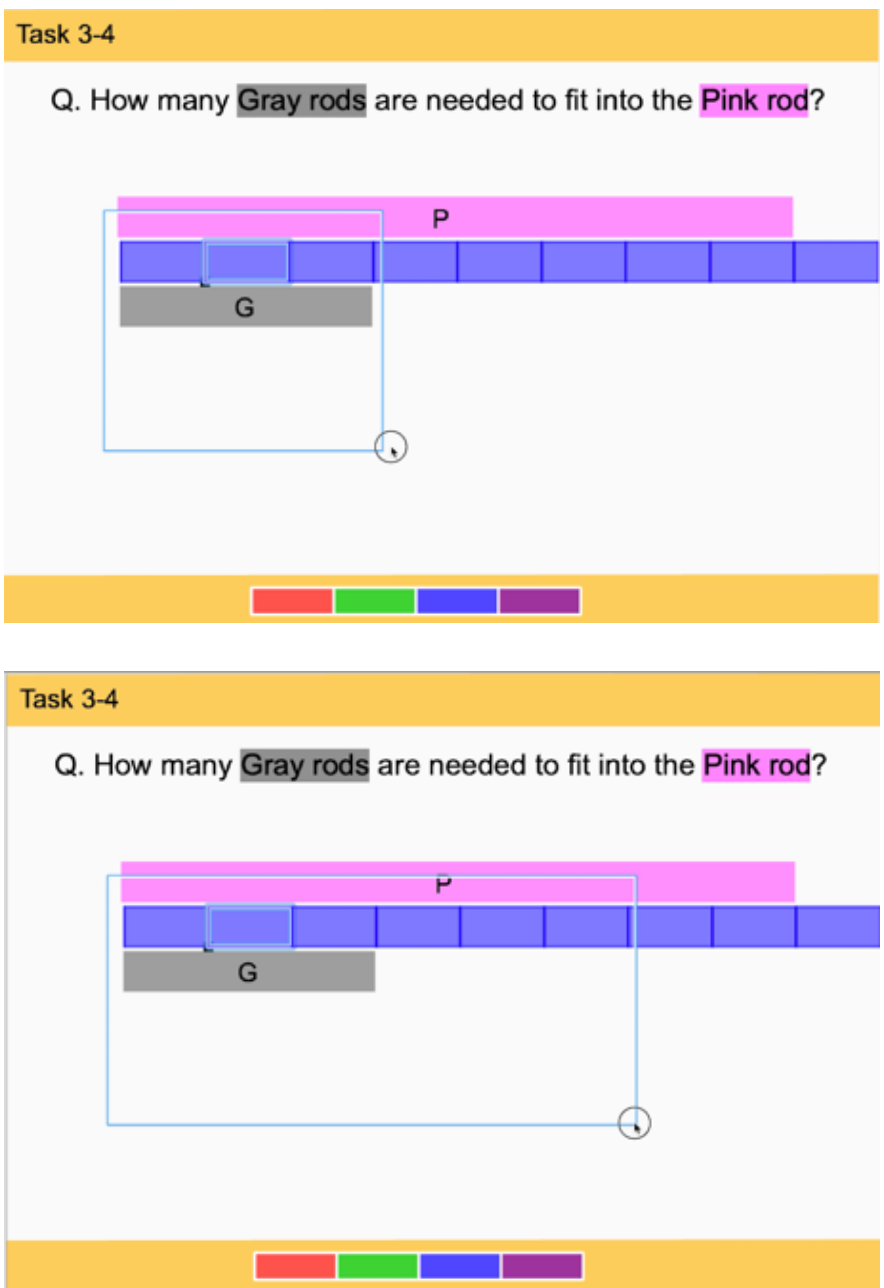




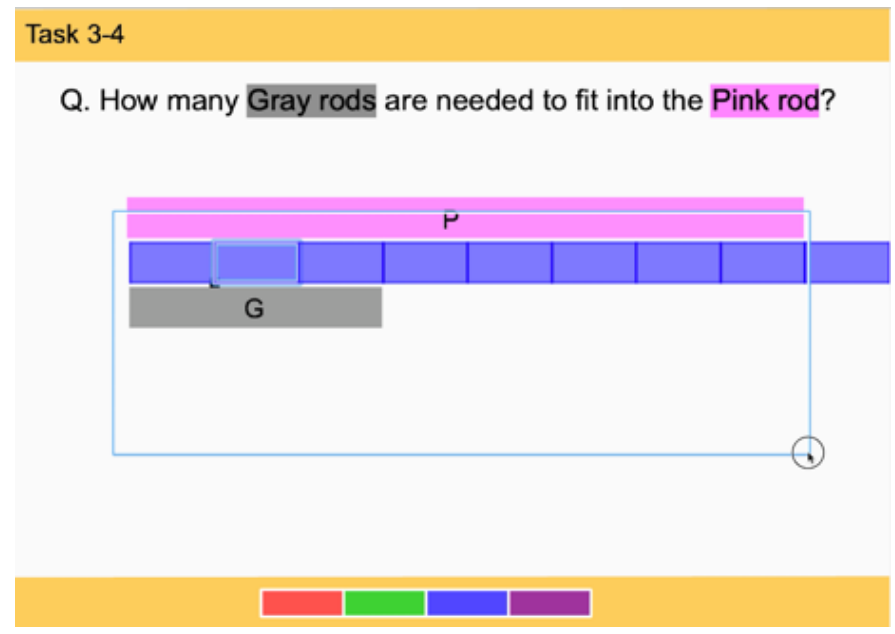

In the type labeled Determining, students could partition a unit length into equal parts and then fit the leftover part using the smaller unit size. The focus of the child in the Determining Type was to determine the relationship between one piece of Dynamic Rulers and an original unit. However, in the Commeasuring Type, students could partition not only the given original unit length but also the quantity to be measured. After coordinating both quantities with the same size of partial units, children could determine the appropriate one-unit size in Dynamic Rulers, compare it to the original gray rod, and measure the length with the newly adjusted unit in the quantity.

If students have already established this type of understanding, they can also think about the inverse relationship, such as, "How much pink rod is needed to fit into the gray rod?" Because they could see the structures of partitioned pieces, the inverse relationships were the same as the reciprocal fractions (e.g., Task 3-4, original measure: $8 / 3$, inverse measure: $3 / 8$ ).

Within the type of Commeasuring, a few students even demonstrated a generalized use of Dynamic Rulers by measuring both quantities with multiple scaled units. Coming to this strategy, children might already have established an idea of how to 
partition a unit length and a quantity with the same partial units. They further anticipated other possibilities for measuring quantities using different unit sizes through multiplicative reasoning.

Gina, a fifth grader, matched the right end line of the gray rod as well as the pink rod using the Blue Dynamic Ruler in Task 3-2 (Figure 38-top) and figured out that the answer was "two and four fifth of gray rods." When asked why she made the unit size of the Blue Dynamic Ruler the length that it was, she explained her intention to "fit" the right end of both rods with a new adjusted unit. She also claimed that the pink rod and the gray one should be partitioned into the same-sized partial units to measure the quantities.

In addition, Gina was trying to "partition into little groups." She could anticipate what she was going to do: "I can partition this into 10 and this [pink rod], too." She adjusted the size of the Blue Dynamic Ruler and matched 10 pieces of the Purple Dynamic Ruler to fit within the gray rod (Figure 38-bottom). She tested her anticipation using the Purple Dynamic Ruler and continued to explain her strategy:

I mean, this is one fifth, and this is two tenth 'cause I partitioned this into 10 groups. 10 pieces. I mean, 10 parts. So, that means two tenths is equal to one fifth. So, I said this [gray rod] is equal to five of one fifth, and it also means 10 of, I mean, kind of one tenth. I mean, yeah. So, you can do the same thing with this. She decomposed the unit length into equal parts, and this subpartitioned unit could measure the given quantities properly. She also had a clear understanding of the relationship between whole and unit fractions (e.g., 5 one-fifths make a whole, 10 onetenths make a whole). Her approach aligned well with her perception of equivalent fractions using multiplication operations. Connecting her memorized facts, she further 
generated $2 / 10$ by multiplying two to the denominator and the numerator of one fifth ("I knew that...five times two which is $10 \ldots$ one times two equals two").

\section{Figure 38}

Gina's Use of the Given Unit

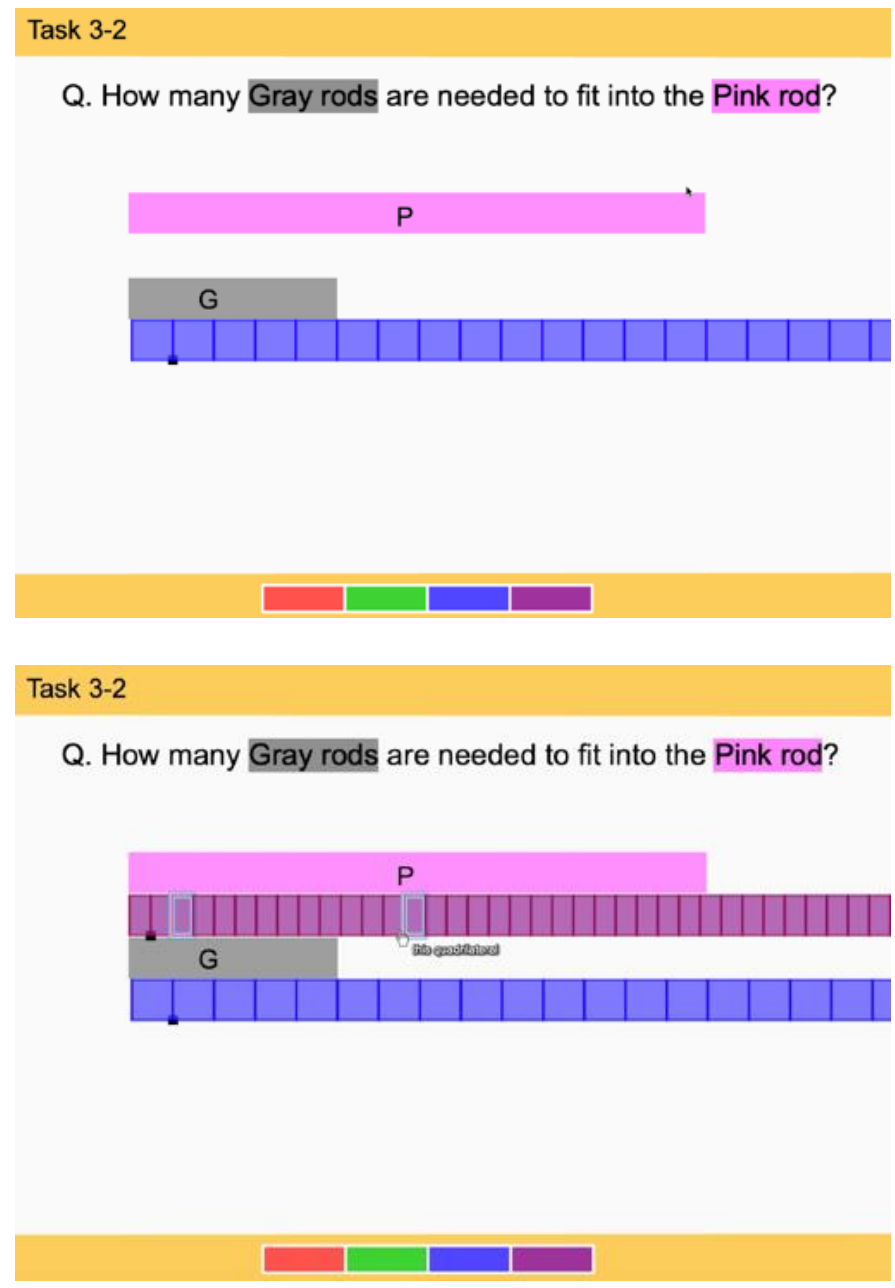

Alex, a fifth grader, tested the different sizes of the Blue Dynamic Ruler to match up its pieces with the gray rod in Task 3-4 (Figure 39-top). He first tried to make the same size with the unit length, but it did not match up with the pink rod: "One section doesn't line up on the edge." Finally, he noticed that thirds could partition the pink and 
gray rods equally: "Three, that's when it first time works." Counting the number of pieces, he concluded the answer was "two and two thirds."

Alex also tried to use a different unit size, a sixth. Because he already proved his understanding of using multiple unit sizes in Tasks 3-2, he could apply a similar strategy to the current task. He conjectured that a sixth would work to solve the task without actual manipulations and operations: "If you do this, you could do this, like, with sixth. Since it's, like, double, you can do that same thing I was talking about earlier, how you can double the number or double the amount since there are eight. It would be 16 if it was, like, split up into six." He had a clear understanding of the relationship between numerators and denominators in a fraction. If there were an equal amount, the fraction notation could vary by the size of units. As the number of partitions of the unit length increased (increasing a denominator), the number of pieces in the measurement increased as well (increasing a numerator). Alex then tested that his conjecture was correct by using the Blue Dynamic Ruler: "If we were to do that just to, like, see if that would work."

\section{Figure 39}

Alex's Use of Partial Units

Task 3-4

Q. How many Gray rods are needed to fit into the Pink rod?

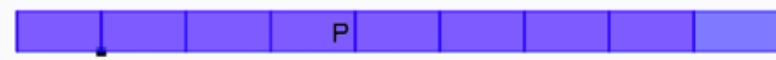




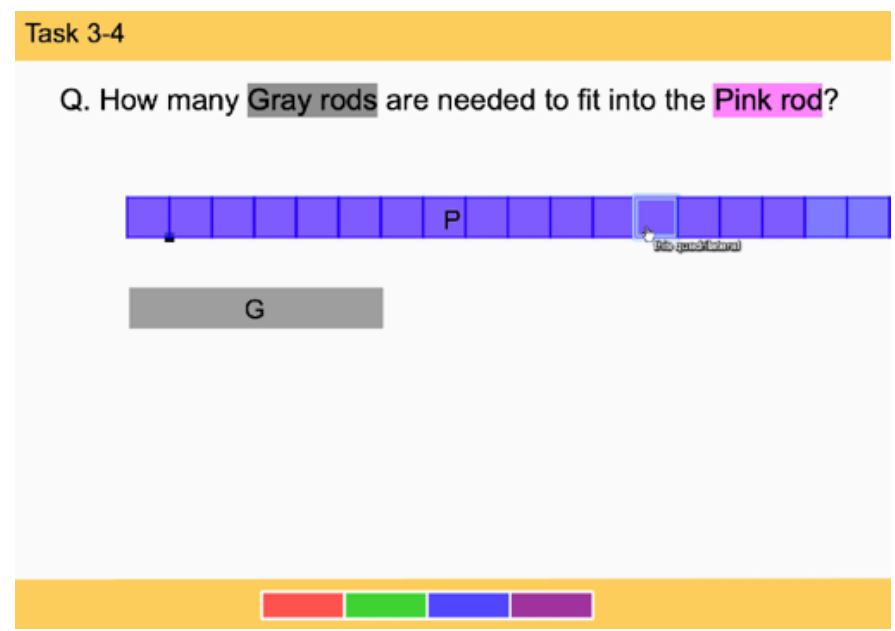

Note. The pink rod overlapped by the Blue Dynamic Ruler, which is why the letter $\mathrm{P}$ appears in horizontal alignment with the ruler.

Children who have experience solving tasks and finding mathematical meanings can extend their strategies further through generalization. The first two examples (Teo, Arim) of strategies were more focused on commeasuring with one unit size. However, these most recent examples demonstrate how some of the students (Gina, Alex) applied to multiple unit sizes. The students made a conjecture in advance to use different unit sizes to measure both quantities and tested the conjecture using the Dynamic Rulers. They explored one of the key mathematical concepts by investigating equivalent fractions, which represented the same value with different fractional notations. Traditionally, students might learn this concept through multiplication or division or by dividing both numerators and denominators. However, by adjusting the size of Dynamic Rulers' units, the students in this study could explore other unit sizes continuously (e.g., thirds, fourths, fifths, and sixths) and make sense of the multiplication operation related 
to measurement (e.g., A half size unit is a half as big to the original size, so it fits in twice as many times. Therefore, I need to double both the numerator and the denominator).

\section{Patterns of Strategy Types Across Fraction-as-Measurement Tasks}

This study's second research question is, "What patterns emerge in children's strategies across the series of the fraction-as-measurement tasks?" To answer it, case study analyses were conducted. It was not surprising that many students showed consistency in how they solved tasks across their interviews. Some students used a strategy type at the beginning of their interview, and they kept using a similar pattern to solve the tasks that followed. However, others approached the series of the fraction-asmeasurement tasks with different types of strategy that they employed. Based on the developed framework, I found various changing patterns in the children's strategy type which emerged from different characteristics of the tasks: within Task Series 2 and 3, across Task series 2 and 3.

This analysis is important because it highlights the affordances and constraints of the tool, and it indicates directions for improvement to the environment and tasks. At the same time, I want to be careful not to claim that using the tool leads to students' learning. It may be that the sequence of tasks elicited increasingly detailed evidence of student thinking, or that repeated use of the tool enabled students to increasingly take advantage of its features, rather than that they developed new mathematical understandings.

The focus of this section is to document how students' strategies varied across the series of tasks. In the previous section, I analyzed children's initial strategies to categorize their strategy types. However, in this section, I further analyze potential factors, including the in-between tasks (i.e., the third task in each series with 
prepartitioned rulers); the prompts asked by me, the interviewer; and my extended probing after students' initial attempts to solve the tasks.

In order to examine how the changing patterns occurred, I identified a critical moment between two consecutive tasks and selected and compared the tasks as the unit of analysis to capture the ongoing transitions (Table 8). Drawing upon the analysis from the analytic memo, I identified representative cases as follows. Within Tasks Series 2, eight children changed types of strategy across Task 2-1 and 2-2 (e.g., Emily - Estimating to Commeasuring) and four children approached differently Task 2-2 and 2-4 (e.g., Sewoong - Estimating to Determining). Within Task Series 3, nine children were engaged in Task 3-1 and 3-2 with distinct strategies (e.g., Fran - Determining to Estimating). Although there was no one who changed the way to solve both Tasks 3-2 and 3-4, some children like Alex solved two tasks with different types of commeasuring (typical to generalized commeasuring). Across Task Series 2 and 3, seven showed changing strategy types between Task 2-4 and 3-1 (e.g., Charim - Determining to Commeasuring). In the following section, I illustrate the cases of shifting strategy types with exemplary episodes.

\section{Table 8}

Patterns of Strategy Types by Task Series

\begin{tabular}{cccc}
\hline Tasks Series & $\begin{array}{c}\text { Changed } \\
\text { Strategy Types }\end{array}$ & $\begin{array}{c}\text { Same } \\
\text { Strategy Types }\end{array}$ & Total \\
\hline Task Series 2 & 8 & & \\
$2-1 \rightarrow 2-2$ & 4 & 21 & 29 \\
$2-2 \rightarrow 2-4$ & & & 28 \\
Task Series 3 & 9 & 20 & 29 \\
$3-1 \rightarrow 3-2$ & 0 & 22 & 22 \\
$3-2 \rightarrow 3-4$ & & & \\
Across Task Series 2 and 3 & 7 & 21 & 28 \\
2-4 $\rightarrow$ 3-1
\end{tabular}




\section{Emily's Case: Within Task Series 2 (2-1 to 2-2)}

Emily, a third grader, was chosen as a case which showed different types of strategy across Tasks 2-1 and 2-2. The main difference between two tasks was the length of the leftover part. In Task $2-1$, the leftover was $1 / 2$ of the original unit (orange rod). On the other hand, in Task 2-2, the leftover was $1 / 4$ of the unit. A half would be the most familiar fraction for children but a fourth requires smaller unit to measure. When solving Tasks 2-1 and 2-2, she showed substantial differences in her strategy types while the Dynamic Ruler tool (Estimating to Commeasuring). In Task 2-1 (Figure 40-top), she measured the size of the orange rod with the Blue Dynamic Ruler and estimated the size of the leftover as a half (Estimating Type). However, in Task 2-2 (Figure 40-bottom), she used fourths to measure the brown rod (Commeasuring Type).

\section{Figure 40}

Emily's Change in Strategy Types, From Unit Length to Partial Units

$$
\begin{aligned}
& \text { Task 2-1 } \\
& \text { Q. How many Orange rods are needed to fit into the Brown rod? }
\end{aligned}
$$

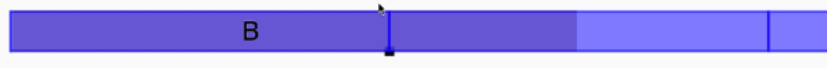




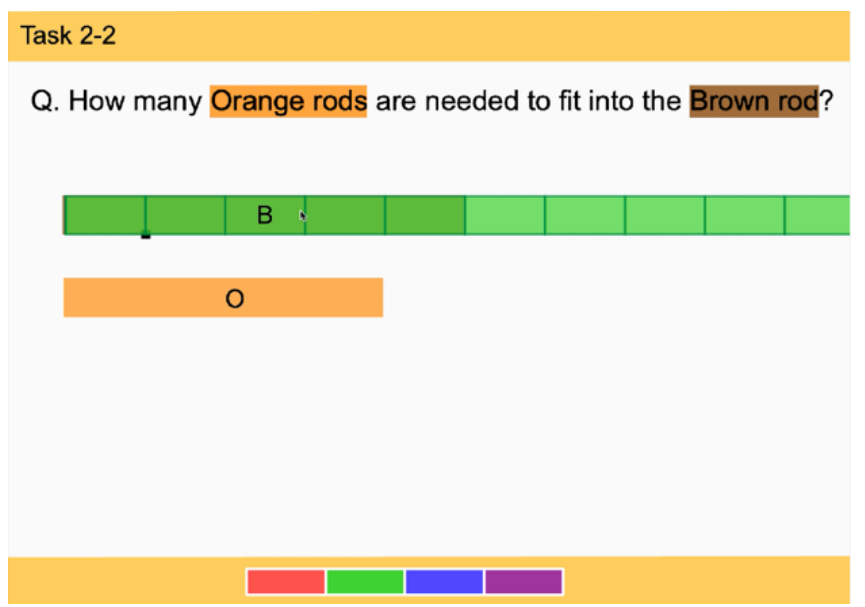

Note. The brown rod overlapped by the Blue Dynamic Ruler in the top image, which is why the letter B appears in horizontal alignment with the ruler. The brown rod overlapped by the Green Dynamic Ruler in the bottom image.

Estimating Type. In the following episode, Emily solved Task 2-1 by comparing the size of the orange rod to the brown rod (Figure 41).

\section{Figure 41}

Emily's Measurement With a Whole Unit and a Partial Unit
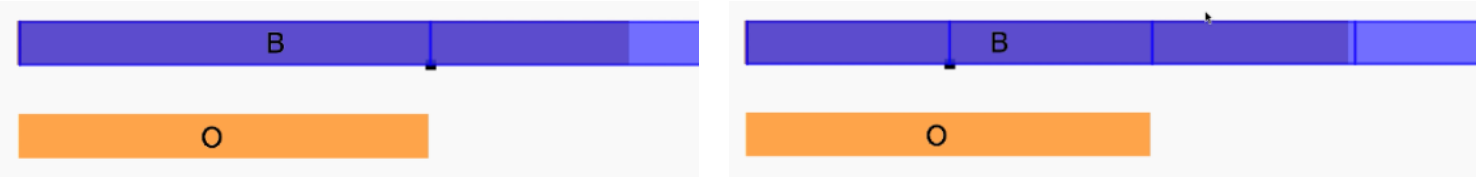

Note. The brown rod overlapped by the Blue Dynamic Ruler, which is why the letter B appears in horizontal alignment with the ruler.

Emily: Uh, one and one half.

Sheunghyun Yeo (Y; i.e., me, the interviewer, researcher, and author of this dissertation): Okay. How did you know one and one half? 
Emily: Because there, this is one whole for the orange, and I tried putting it in a brown but in a whole entire another one. But it fit, but it was pretty much almost exactly halfway in the center. So, I just rounded it up to halfway and, so, one and one half.

Y: Can you convince me, too. Is it a half of orange? You can use the different color.

Emily: Like again by doing this. I make the next line go right there. Then I put it on this, then, since these are all a half. Then, um, this is one, and this is one half.

Y: How many halves are there then?

Emily: Three halves.

Y: Okay. Then the first answer you just said is like one and a half. One and a half and three halves are the same?

Emily: Yeah.

Y: Why are they the same?

Emily: Because two halves make a whole. (Taken from Emily's transcript. This episode appears on Lines 151-174 of the transcriptions. Future notations such as this one will be abbreviated in this format: "Emily, 151-174.")

Emily approached this task similarly to how she approached previous tasks (Task Series 1), by measuring the original unit length (orange rod). However, Task 2-1 included a leftover, which was a portion of the brown rod that was of an indeterminate size less than one orange rod. Therefore, Emily estimated the size of the leftover and expressed it 
as a half (Figure 41-left). When asked to confirm that the size of the leftover was a half, she adjusted the size of the Blue Dynamic Ruler's units (Figure 41-right) and matched two blue pieces with the length of the orange rod ("I make the next line go right there. Then I put it on this, then, since these are all a half."). She also demonstrated her understanding of the equivalence between a mixed number $\left(1 \frac{1}{2}\right)$ and an improper fraction (3/2): "Because two halves make a whole."

In terms of unit coordination, Emily's measurement strategy changed from single level to composite level. When she used the orange rod as the unit of measure and found its relationship to both the brown rod and the Blue Dynamic Ruler, she was able to measure one by one through the Blue Dynamic Ruler. However, when she used half the size of the orange rod with the Blue Dynamic Ruler, the shorter blue piece was the smallest unit size (a half), and the orange rod was a composite unit $(1=2 / 2)$ comprising two blue pieces. She further measured the brown rod with the blue pieces $(3 / 2)$ and demonstrated the concept of equivalence between different fractions verbally. Emily's unit coordination was more sophisticated in the following episode.

\section{Figure 42}

\section{Emily's Measurement With Other Partial Units}
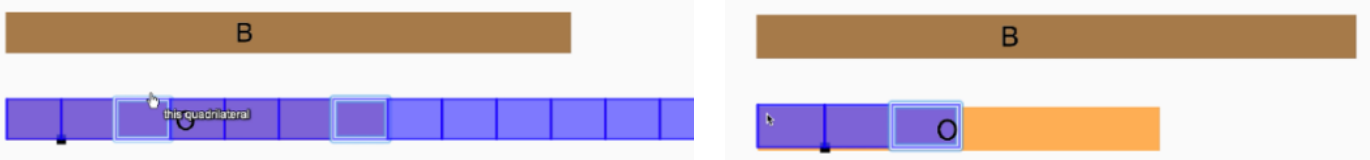

Note. The orange rod overlapped by the Blue Dynamic Ruler, which is why the letter $\mathrm{O}$ appears in horizontal alignment with the ruler. 
Y: Can you try to use a different size?

Emily: Different size? Hmm.

Y: It could be much longer. It could be much shorter.

Emily: I'm going to make this [an] even number.

Y: Can you tell me more? Why you are trying to make it even?

Emily: Because if I make it an odd number, then there will be a half of that hanging off, and I won't know the exact answer, and I won't know if that's an exact half. Yup. I still got three halves.

Y: How much of this, one piece of the blue?

Emily: That's one third.

$\mathrm{Y}$ : One third of? Can you say one more time?

Emily: It's like, it's, um, that piece is one third of a half but one sixth of the whole.

Y: One third of one half is one sixth of the orange rod?

Emily: Of the whole. Yeah.

Y: Of the whole? Why is that one sixth? One third of one half is one sixth?

Emily: I'd like to imagine, like, a candy bar. So, if you have a candy bar that has, like, six little parts into it. Then the whole piece is just the candy bar. Then it's divided into six parts. So, each one of those pieces is a sixth. But you break it exactly in half. Um, and then, so, you have two halves. There's 
three in one half. So, it's one third of that part. Like, if that was the new whole, then there would be one third of that half of the whole.

Y: So, can you also use that strategy to explain with the Dynamic Ruler? So, if this is one candy bar, how can you explain that one third of one half is one sixth? Can you click here?

Emily: Yeah.

Y: So, if this is one candy bar, like the orange rod, how can you explain one third of one half is one sixth?

Emily: So, I'm going to break this half. This is my half of the candy bar, and this part of the half of this was the whole. Then this is one third of it. Then this is the whole, so this piece is now one second. But if we broke it in half again, they don't see one third. One third. (Emily, 175-210)

This exchange began with my question about other possible sizes of units. Emily matched seven pieces of the Blue Dynamic Ruler with the orange rod (Figure 42-left). However, she realized that an odd number of pieces did not work because the pieces could not be equally partitioned if she had an odd number of them. Then, she partitioned the orange rod into six pieces (Figure 42-right) and measured the brown rod with sixths (i.e., "one third of one half"). Interestingly, she demonstrated an understanding of a partial unit of a partial unit (fraction of fraction). When asked about the sixth, she was reminded of the candy bar analogy and explained how she could get a sixth with subdivision. I also asked her to use a Dynamic Ruler representation to elicit the same meaning. She explained that a half of the orange rod was three blue pieces, and a sixth 
was one of the three blues that constitute a half. This explanation had three types of unit structure - one blue (one sixth of the orange rod), three blues (one half of the orange rod), and one orange $($ six of one blues $=$ two of three blues $=$ one $)$. To measure one brown rod, Emily combined the concepts in these three structures: three of one sixth makes one half; three of one half makes $3 / 2$. This size (one sixth) was not achieved with a fraction that is common among third-grade students. Other students in grade 3 were more familiar with fractions that include denominators as a power of two (e.g., 1/2, 1/4, 1/8), but Emily used one third and one sixth in Task 2-1.

Furthermore, in this exchange, the role of the tool was slightly changed from exploring mathematical relationships to demonstrating mathematical knowledge. In the beginning of the tasks, Emily matched the Dynamic Ruler to make the unit length and figured out the leftover part as a half of the unit. However, in the latter case, she used the tool to represent and check her understanding of unit relations.

Commeasuring Type. In the following episode, Emily solved Task 2-2. She did not match the unit length with an existing piece on a Dynamic Ruler but, instead, adjusted the ruler's black dot to make a partial unit size that would measure both the given unit and the given length (Figure 43).

\section{Figure 43}

Emily's Measurement With a Partial Unit 
Note. The orange rod overlapped by the Green Dynamic Ruler, which is why the letter O appears in horizontal alignment with the ruler.

Emily: One and one fourth.

Y: Um, what I am curious is, like, when you find one piece of this size, you're trying to change the size, but you seem to be, like, looking for something.

Emily: How I decided was [that] I was trying to make these two lines. [Emily aligns the edges of the brown and orange rods with the edge of the Green Dynamic Ruler.] If there was another line in between [that could] line up so that I knew that ... there would actually be a correct amount of chunk that would fit into that, that would be a certain amount of these pieces. But let's say I just did it like that; there isn't a line lining up, so I won't know if it's exactly, like, a half or what. So, that's why I carefully decided that it had to be like that. And also, this line also matched up with the end of that. So, then it would be easy for me to know that there were four pieces in one, but then there was one extra that would fit into the brown, too. (Emily, 223228)

Because the default size of a piece of the Green Dynamic Ruler was shorter than the orange rod, Emily explored the different sizes that the unit could be, from halves of the orange rod to thirds to fourths (Figure 43). She explained how she found the appropriate size for the green pieces: "I was trying to make these two lines." She actually lined up the right edge of the brown rod with the edge of the orange rod and aligned both with the edge of one of the ruler's units ("And also this line also matched up with the end 
of that."). This alignment allowed her to recognize how many pieces of the Green Dynamic Ruler needed to fit into the brown rod ("It would be easy for me to know that there were four pieces in one, but then there was one extra that would fit into the brown.").

In this episode, Emily's unit coordination was demonstrated through her manipulation and explanation. Changing the size of the Green Dynamic Ruler's pieces, she seemed to unitize a piece as a new partial unit to measure the orange rod and the brown rod. Simultaneously, she aligned the Green Dynamic Ruler with both the brown and orange rods. When she dragged the black dot to change the size of the Green Dynamic Ruler's units, she was actually looking for a way to partition the brown rod and the orange rod at the same time with the same unit size. This commeasuring was why a half or a third would not work (i.e., it could not partition both rods). A half of the orange rod or a third could partition the orange rod but could not appropriately partition the brown rod using full unit lengths. Therefore, when she found a fourth as a new unit of measure, she could partition both rods: the orange rod was four fourths and the brown rod was five fourths. Through her interpretation of the manipulated representations, she could fully understand the relationships between units in measuring the brown rod with a partial unit size ("There were four pieces in one, but then there was one extra.").

Sewoong's Case: Within Task Series 2 (2-2 to 2-4)

Sewoong, a third grader, was a representative case of children who solved Tasks 2-2 and 2-4 differently. Task 2-2 involved $1 / 4$ and Task $2-4$ involved $1 / 3$. Because children tend to experience difficulty to represent $1 / 3$ with appropriate terms or symbols, Sewoong struggled a little in the latter task. Here, his strategy type changed between two tasks 
(Estimating to Determining). In Task 2-2, (Figure 44-top), Sewoong measured the length of the orange rod by making the pieces of the Green Dynamic Ruler the same size and estimated the leftover part as a half (Estimating). However, in the next task, Task 2-4 (Figure 44-bottom), he initially made the pieces of the Green Dynamic Ruler the same size as the orange rode, then partitioned one green piece evenly into four red pieces using the Red Dynamic Ruler. To measure the additional difference between the green piece and the red piece, he used the Blue Dynamic Ruler by partitioning the red piece into four blue pieces (Determining Type).

\section{Figure 44}

Sewoong's Unit Partitioning

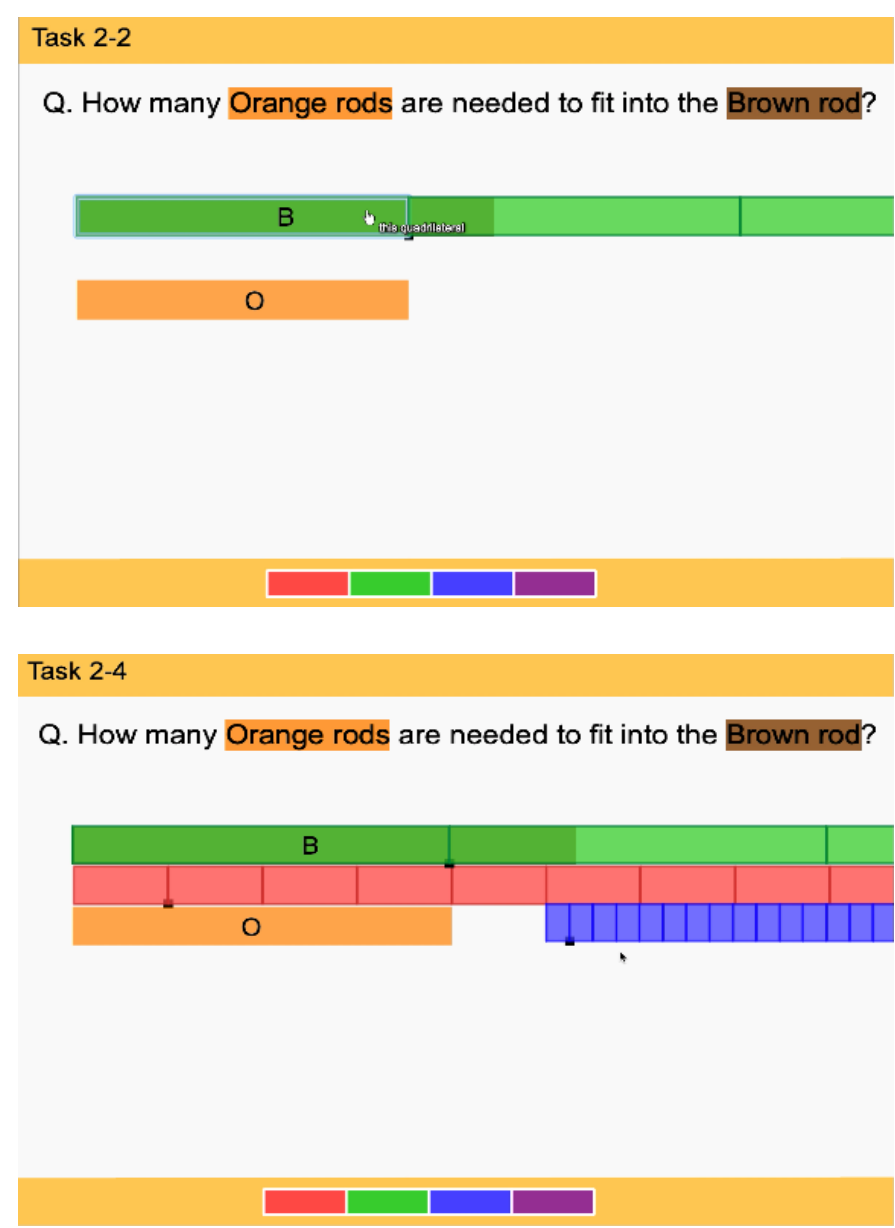


Note. The brown rod overlapped by the Green Dynamic Ruler, which is why the letter B appears in horizontal alignment with the ruler.

Estimating Type. In the next episode, Sewoong solved Task 2-2 by measuring the brown rod with the Green Dynamic Ruler, in which the pieces were the same size as the orange rod (Figure 45).

\section{Figure 45}

Sewoong's Method of Checking the Leftover Length With an Additional Dynamic Ruler
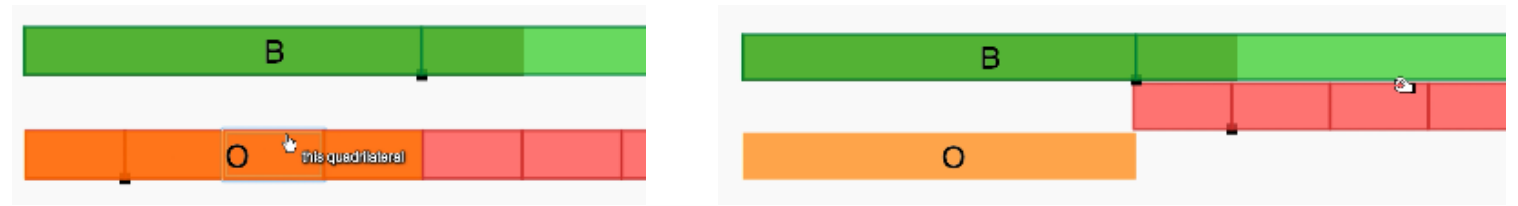

Note. The orange rod overlapped by the Red Dynamic Ruler in the image on the left, which is why the letter $\mathrm{O}$ appears in horizontal alignment with the ruler.

Sewoong: One and a quarter.

Y: Can you tell me more? How did you get that? One and a quarter of what?

Sewoong: So, one and a quarter of orange.

Y: Okay. So, can you tell me? How did you get that?

Sewoong: Wait, let me check. Yeah, it's a one and a quarter, I think.

Y: Hmm. How do know this is a quarter of the orange? 
Sewoong: Because this is the half of orange rods and then there's two, two quarters in the one half. So, this is one and, and the quarter.

Y: Okay. Can you use like a red ruler to show this is a quarter?

Sewoong: What's red?

Y: Red ruler. So, do not delete that. Yeah, you can use this tool and then know this much is a quarter of the orange. I'm just curious about that relationship. How can you make me convinced that?

Sewoong: Like this. So, this is a half and then one quarter.

Y: Okay. Then this is one quarter too?

Sewoong: Yeah. Oh yeah.

Y: How?

Sewoong: You can use it to check it. Yes. (Sewoong, 376-404)

It was not surprising when Sewoong made the orange rod and a piece of the Green Dynamic Ruler the same size because he utilized the same strategy in Task 2-1. He explained how he got the quarter size by halving the green piece and halving the half of the green piece. He was able to estimate the leftover size as a quarter ("one and a quarter"). When asked to verify the size of the leftover with the Red Dynamic Ruler, he created the length of one red piece by partitioning the orange rod into four pieces (Figure 45). He indicated that two red pieces were half of the orange rod, and one was a quarter. And Sewoong matched the leftover size and one red piece's size by placing the Red Dynamic Ruler below the green one ("You can use it to check it"). 
Determining Type. Even though he estimated the leftover part with the Green Dynamic Ruler, Sewoong demonstrated his further understanding of how to measure the leftover that equals a partial unit size by using the Red Dynamic Ruler. This partial unit concept arose from the size of the leftover. Because he already knew the size of the leftover was a quarter by estimating the size of the remaining part with double-halving (partitioning a half into two parts), he seemed to decide to directly partition the orange rod into four quarters. Such a partitioning idea was evidenced even more during the following episode in Task 2-4 (Figure 46).

\section{Figure 46}

Sewoong's Subsequent Partitioning
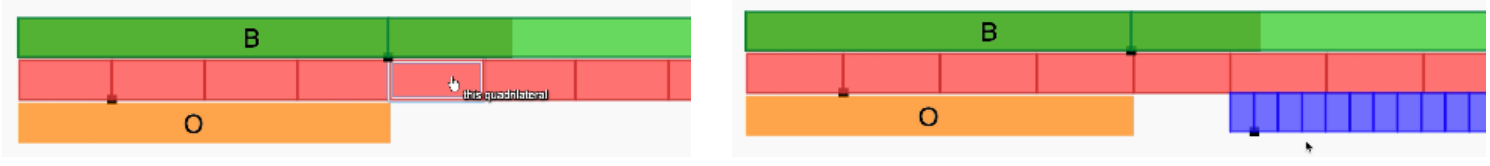

Sewoong: Wait, what is - I need to check. I need to check the quarter. Hmm? Did I even measure it right? Yes, I did. What is the-Oh wait, I think I know. One quarter and one and the half of quarter- One half - I mean one full quarter and one and a quarter of a quarter.

Y: One and a quarter of a quarter. Okay. Why do you think this is-

Sewoong: Wait, I want to check if that's right. What is the quarter of a quarter?

Y: Yeah. I am curious about it. 
Sewoong: How long is it? Oh my. Nope. Wait, is that right? No, no. Oh, I think I know. Four quarters and a quarter of a quarter and the quarter.

Y: How many quarters are there?

Sewoong: Look, look. So, four quarters equal one orange rod. And then one quarter. Plus, I just did four quarters of the quarters. And then I just look at it, and then it kind of looks like there's a quarter in a quarter of a quarter.

Y: Interesting. Why did you use the third ruler?

Sewoong: This. To check how many quarters there are, I mean, how- To check how long the quarter is in the quarter. (Sewoong, 442-458)

Sewoong changed the size of the Green Dynamic Ruler so that one unit on the ruler was the same size as the orange rod, as he did in previous tasks (2-1 and 2-2). Then, he pulled out the Red Dynamic Ruler to measure the leftover part (Figure 46-left). While he usually used additional Dynamic Rulers when I asked him to use those for probing, this was the first time he used multiple Dynamic Rulers of his own volition to figure out the leftover length more precisely. Sewoong used fourths to measure the leftover like in previous tasks, but it was not enough to measure the new leftover. Therefore, he decided to iterate the same strategy to measure the difference between the leftover and one red piece by using the Blue Dynamic Ruler (Figure 46-right). He partitioned a fourth of the orange rod into four pieces and generated a unit on the Blue Dynamic Ruler that was 1/16 the size of a unit on the Green Dynamic Ruler. Finally, his solution for the task was the sum of three different unit sizes (green segment: one whole, red segment: one fourth, blue 
segment: 1/16). The expected solution was $4 / 3$ (1.3333), but his answer was close enough given the limitations of the Dynamic Ruler environment $(1+1 / 4+1 / 16=21 / 16=1.3125)$.

When Sewoong first noticed the leftover between two rods, he used the Red Dynamic Ruler in Task 2-4. He subsequently used the Blue Dynamic Ruler to measure the new leftover between the brown rod and five red pieces. He drew three different unit sizes with a full understanding of the relationship: green equals one, red equals a quarter, and blue equals a quarter of a quarter. And this reciprocal relationship was based on the understanding of the whole and fractional units ("four quarters is equal to one orange rod").

In the following exchange, from his work on Task 2-2, he seemed to feel it was necessary to use partial unit sizes instead of the same-sized unit length.

Y: Then, which ruler do you prefer using to get the number of orange rods between blue, green, and red, and why?

Sewoong: Red.

Y: Why?

Sewoong: Because the quarters are easier to see, because if you don't see the quarters, it'll be like, what is this?

Y: That is a question. What is that? The answer on this problem.

Sewoong: If you go like that, you see, you'll see what the quarter is, but if you only see the green, it's gonna be way longer. So, you'll be like, what? What? Like.

Y: It's hard to figure it out, right? 
Sewoong: Yeah. (Sewoong, 426-440)

Between the Green Dynamic Ruler (with one whole piece equaling the length of the orange rod) and the Red Dynamic Ruler (four red pieces partitioning the length of the orange rod), Sewoong selected the Red Dynamic Ruler (Figure 47), because he was wondering how to measure the leftover part with the whole size unit ("Because the quarters are easier to see, because if you don't see the quarters, it'll be like, what is this?"). Even though he was able to estimate the size of the leftover, it might not have been enough to solidify his concept of partitioning. His approach could have triggered the use the of partial units with subsequent partitioning in the following task (2-4).

\section{Figure 47}

Sewoong's Comparison Between the Given Unit Size and the Equally Partitioned Size

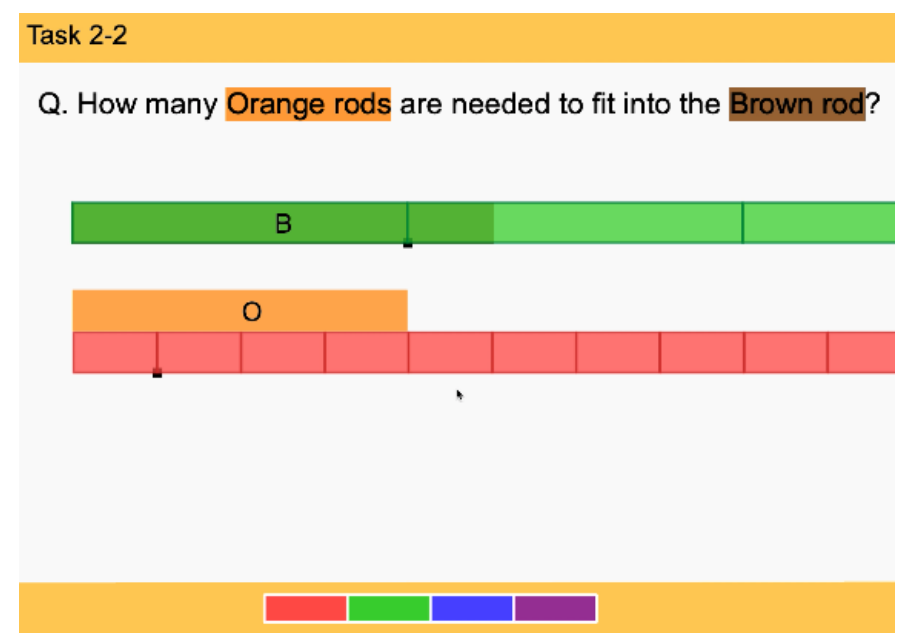

\section{Fran's Case: Within Task Series 3 (3-1 to 3-2)}

Fran, who was a third grader, used Dynamic Rulers with intermediate units in Task 3-1 (Determining), but changed to using estimation to figure out the size of a leftover length in Task 3-2 (Estimating). The size of the leftover in Task 3-1 was $3 / 2$ of the 
gray rod but the size was getting longer in Task $3-2$ as $7 / 4$ of the gray rod. Both improper fractions might be not easy but $7 / 4$ would be more difficult to estimate by eyeballing, which requires more cognitive demanding. In Task 3-1 (Figure 48-top), she adjusted the size of the Blue Dynamic Ruler to match the length of the gray rod, then measured the leftover part with the Purple Dynamic Ruler as an intermediate unit (Determining Type). However, in Task 3-2 (Figure 48-bottom), she only matched the Blue Dynamic Ruler with the size of the gray rod and estimated the leftover part with the same unit length size (Estimating Type).

\section{Figure 48}

Fran's Change of Strategy Types from Determination to Estimation

\section{Task 3-1}

Q. How many Gray rods are needed to fit into the Pink rod?

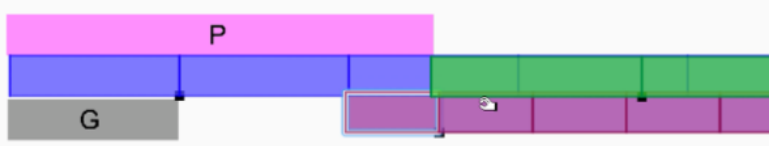




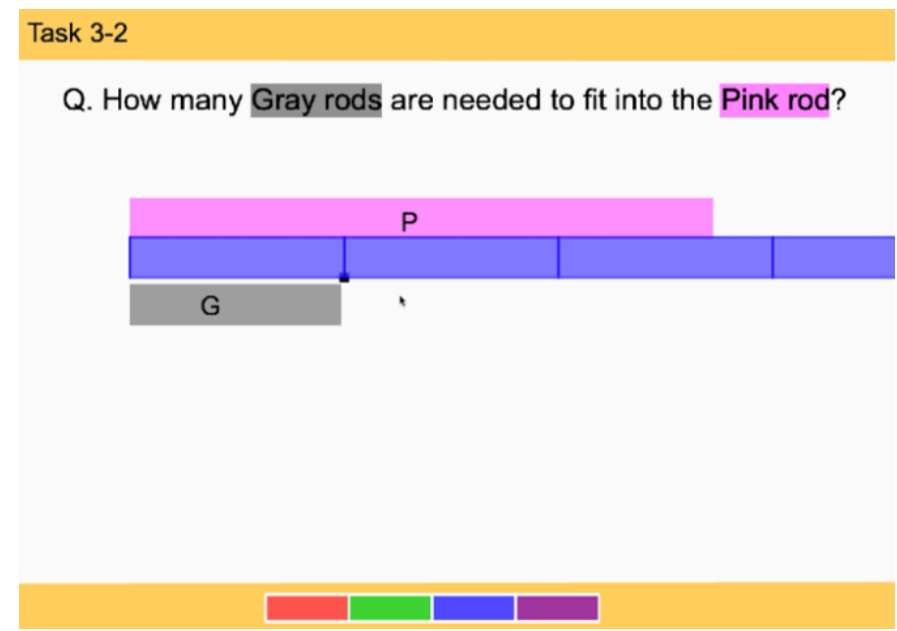

Determining Type. In the following episode, Fran solved Task 3-1 by using three different Dynamic Rulers to find out how many gray rods fit in the pink rod (Figure 49).

\section{Figure 49}

Fran's Measurement With an Intermediate Unit

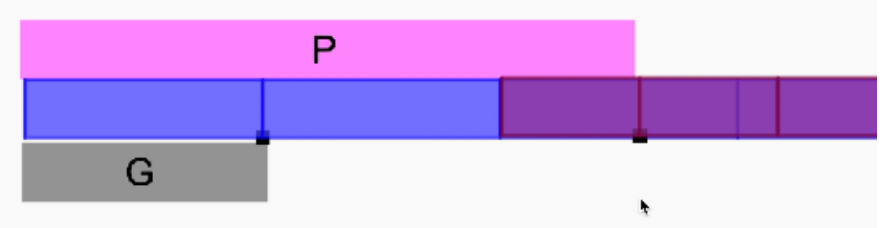

Fran: Let's see how much purple. Yeah, I think that is - Oh, I think I know. I think it's, um, two and a half.

Y: Two and a half of what?

Fran: Two and a half of the gray. Two and a half of the gray rods are needed to fit into the pink rod.

Y: Okay. I think you did some interesting stuff. So, can you tell me, um, your process of thinking? 
Fran: I did [Fran indicates the middle of the third blue piece]. So, first I measure this one small line there.

Y: Why do you overlap the two different rulers?

Fran: Because I overlapped because I can. If I just do it over here, it wouldn't be, if it wouldn't, it would be too hard because these are the way the two lines are there. So, I did. I was trying to make a half, so it's about the same size. So, then it's half, not a quarter.

Y: So, how do you know this is a half of — half size? And half the size of what?

Fran: Size of the rod. The half size of the-

Y: Which half?

Fran: Half size — Oh, the gray.

Y: Okay. Why do you think it is the half of gray?

Fran: Wait, half of the, um, half of the blue two lines.

Y: And is that same as the half of the gray?

Fran: Yeah. Because it's the same size of the gray.

Y: Okay. And how do you think, uh, this is, uh, a half of that?

Fran: I think it's half of that.

Y: How did you compare that? 
Fran: I can compare it because I could compare it to, to just like a normal size, but then I would just make it smaller a little bit. Like all the ways small. It's too small. (Fran, 351-384)

After Fran manipulated the tool to make the pieces of the Blue Dynamic Ruler the same size as the gray rod ("So, first I measure this one small line there."), she covered the third blue piece with the Green Dynamic Ruler. The colors of the two Dynamic Rulers were translucent so that she could see the right edge of the blue piece through the Green Dynamic Ruler. The edge of the third piece on the Blue Dynamic Ruler did not match with the right edge of the pink rod, so she explained that it would be difficult to measure the leftover part with the Blue Dynamic Ruler ("It would be too hard because these are the way the two lines are there."). By overlapping two Dynamic Rulers, she seemed to recognize that the leftover part was a half of the gray rod. Finally, she measured the leftover with the Purple Dynamic Ruler to check that the size of the leftover was a half of the unit length. She thought a purple piece was a half (Figure 49) because the purple piece was shorter ("make it smaller a little bit") than the blue piece ("normal size").

Because Fran used an intermediate unit (a half), made with the Purple Dynamic Ruler, to check the relation between the leftover and the blue piece, her strategy type was categorized at the Determining Type. Apparently, she measured the leftover with the Purple Dynamic Ruler and compared it to a piece of the Blue Dynamic Ruler. She explained the purple piece was a half of the gray (or blue) rod, but actually, the size of one purple piece was not a half. In Figure 49, it is possible to see the edges of the third blue piece through the second purple piece, and two purple pieces do not fit into one blue. While Fran measured the leftover length of the pink rod with the Purple Dynamic Ruler, 
she seemed to estimate the purple piece's size compared to a blue piece, imprecisely measuring with the partial unit size.

Estimating Type. In Task 3-1, Fran seemed to reason that the fraction was one half of the gray rod by measuring with an additional Dynamic Ruler. However, the same strategy seemed difficult to apply to the new situation presented in Task 3-2, where she had to identify a fraction that was three quarters of the gray rod. In the latter task, the leftover amount was not a fourth but three fourths. What she figured out was that a quarter of the third piece of the Blue Dynamic Ruler extended beyond the right end of the pink rod. In the following episode, Fran changed how she reasoned the leftover's length in Task 3-2.

\section{Figure 50}

Fran's Measurement With a Given Unit Length and Partial Unit
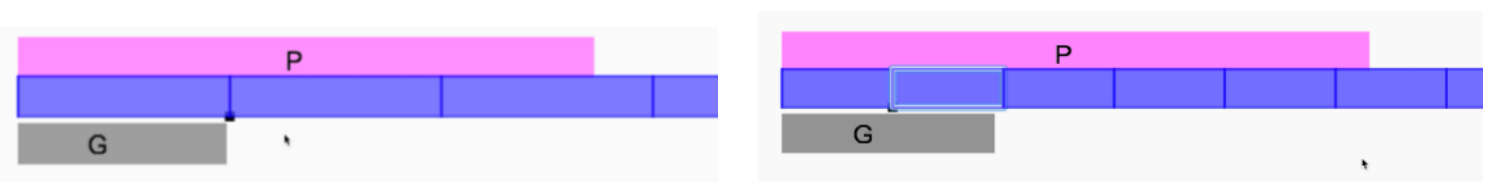

Fran: Let's see. Maybe. I think two and a-Well, the hard thing is because there's quarter on the other side.

Y: Where's the quarter?

Fran: The quarter's right there. It's in the end.

Y: Okay.

Fran: That's a little hard. 
Y: Then you can explain all your own way to- Not exactly the number, but you can express that with the words.

Fran: I think it's like about a quarter but just at the end. So, like, it's like half and a quarter, I think.

Y: Okay. That's very interesting. So, half and-

Fran: Half and like a little bit more. It's like about half and like a little bit more than a half.

Y: Okay. It would be half, half and a little more, or it could be half and a quarter, you said.

Fran: Yeah. I think it's half and a little more.

Y: Okay. Um, can you use a different size to show that?

Fran: Make two half of the this. So, that'd be one, two. Ah, so then if I-That's a half. So, then it's like a little bit more. (Fran, 387-412)

As she placed the Blue Dynamic Ruler under the gray rod, she adjusted the ruler's unit size to be the same size as the gray rod (Figure 50-left). She could estimate the difference between the pink rod and the third blue piece, estimating it as a quarter ("The hard thing is because there's quarter on the other side."). The measuring result would be three blue pieces if not for the leftover portion of the third blue piece—which was roughly a quarter-piece in length and extended beyond the pink rod. When asked to express the measurement with mathematical terms or notations, she estimated the leftover as the sum of a half and a quarter ("So, like, it's like half and a quarter, I think."). She also tried to use a half-sized unit (Figure 50-right). She measured the pink rod with a 
shorter blue unit, which was a half of the previous unit size. She partitioned the gray rod into two pieces ("Make two half of the this.") and figured out the number of blue pieces by counting two pieces ("So, that'd be one, two."). Then, she counted the fifth blue rod as a half ("That's a half.") and recognized the new leftover (“So, then it's like a little bit more."). However, she did not precisely partition the gray rod into two pieces of the Blue Dynamic Ruler. As a result, she could not achieve a quarter precisely, which should have been half the size of a blue piece. These imprecisions might have prompted the children to estimate the leftover part rather than use the Dynamic Ruler tool to verify the unit relations.

\section{Alex's Case: Within Task Series 3 (3-2 to 3-4)}

In this study's strategy type framework, I want to distinguish two subcategories of the Commeasuring Type: typical and generalized. Some of the study's subjects achieved the Typical Commeasuring Type, while others demonstrated that they could generalize their fraction understanding, meaning they could attain the Generalized Commeasuring Type. In the following section, I explain how Alex, a fifth grader, changed strategy types from Typical Commeasuring to Generalized Commeasuring in his fraction understandings through the use of the dynamic tool when solving Tasks 3-2 and 3-4. The leftover length in Task 3-2 is 7/4 of the original unit (gray rod) and the leftover in Task 3-4 is $5 / 3$. In Task 3-2 (Figure 51-top), Alex partitioned the gray rod into six pieces with the Blue Dynamic Ruler and measured the pink rod with a partial unit (Typical Commeasuring Type). Furthermore, in Task 3-4 (Figure 51-bottom), he first partitioned the gray rod into three pieces, then measured the pink rod with a third. Anticipating 
different fractions by doubling, he also proved that sixths could be a unit for equivalent fractions (Generalized Commeasuring Type).

\section{Figure 51}

Alex's Change of Strategy TypesFrom a Single Unit to Multiple Units
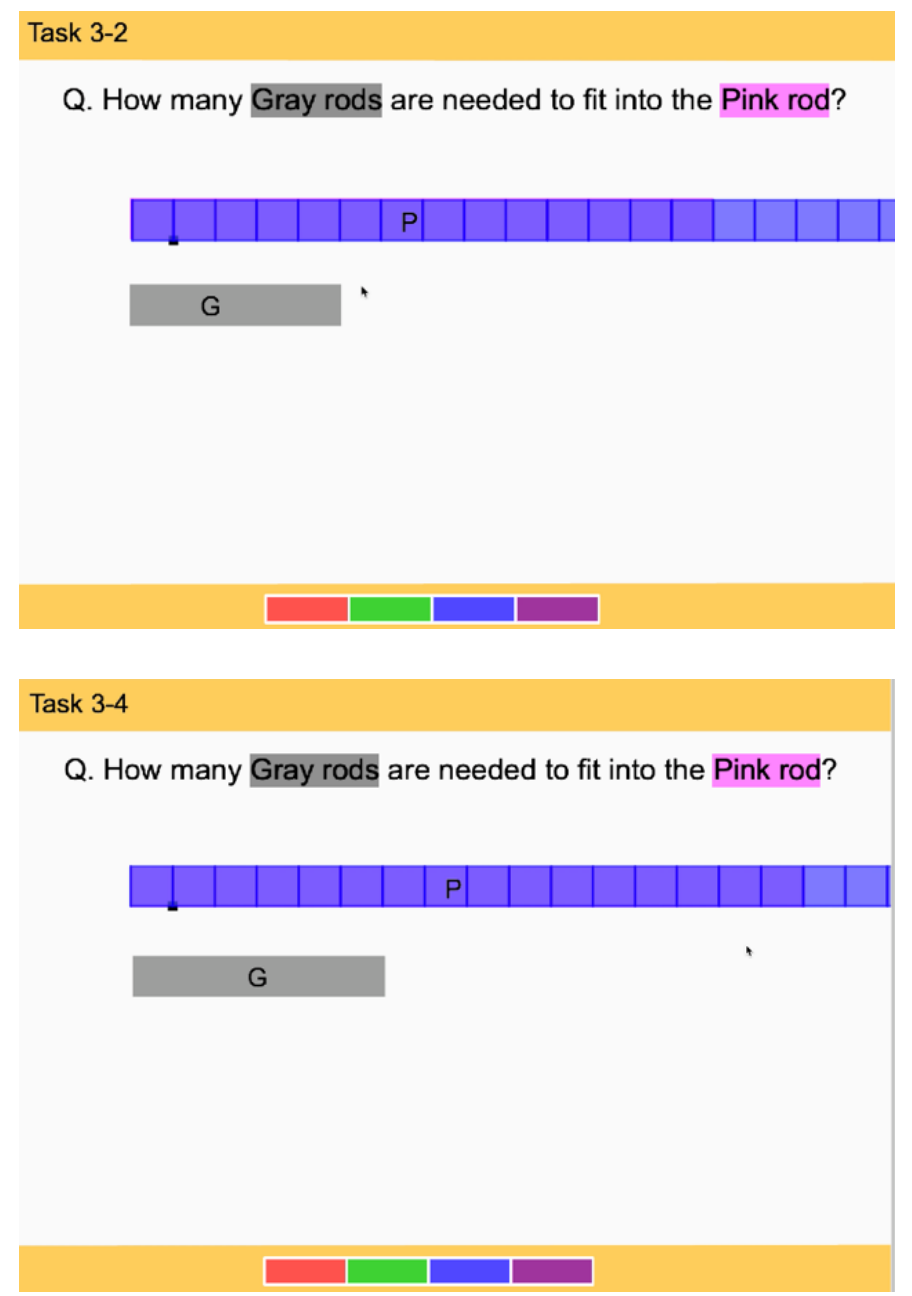

Note. The pink rod overlapped by the Blue Dynamic Ruler, which is why the letter $\mathrm{O}$ appears in horizontal alignment with the ruler.

Typical Commeasuring Type. In the following episode, Alex solved Task 3-2 and explained his thinking process (see Figure 52). 


\section{Figure 52}

Alex's Measurement With a Partial Unit

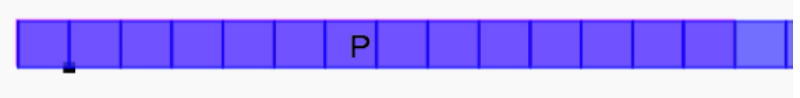

G

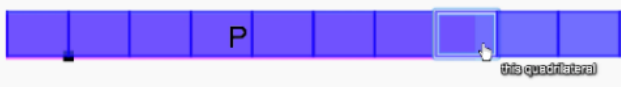

G

Note. The pink rod overlapped the Blue Dynamic Ruler, which is why the letter P appears in horizontal alignment with the ruler.

Alex: So, we'll look at those. We have to align with the edge up. The edge of the blue rod up to the edge of the gray rod, the left edge. So, we have to, umlet's do one whole. That does not line up. We'd go to halves. Still does not line up. Um, thirds. Nope. Fourths. No. Fifths. It's, um, when we go to fifths, that's when it first works. Then, it is sixths. Um, and then, so, when we get to fifths, that's when it first works. We take the blue, line it up. Um, one up, this is one of the grays; this, two of the grays; this, um, this would be another one of the grays, but it's only four fifths of - So, um, we get to one, two, and then four out of the five because five of these blue sections are equal to the one in the gray rods.

Y: Then how many total?

Alex: Um, there would be, um, 14 blue sections, but that would be two and four fifths gray rods. (Alex, 560-572)

Alex demonstrated the Typical Commeasuring Type by partitioning the given unit length (the gray rod) into fractional amounts (fifths). Initially, he made the Blue Dynamic Ruler's pieces the same size as the gray rod. However, he realized the segments did not 
match up with the right end of the pink rod ("Let's do one whole. That does not line up.”). Therefore, after adjusting the size of the Blue Dynamic Ruler, he decided to make its units smaller and dragged the black dot to achieve blue pieces that were a fifth of the size of the gray rod (Figure 52-left; "Fifth. It's um, when we go to fifths, that's when it first works.").

Alex seemed to partition the unit length (the gray rod in Figure 52) and the object being measured (the pink rod) at the same time. It appears that Alex conceived of commeasuring because he subdivided the given unit and the quantity simultaneously with an adjusted unit of the Dynamic Ruler. In Task Series 2 and 3, the whole size unit (the bottom rod in each task) was not precise as a measure of the longer rod (the top rod in each task). This intentional imprecision motivated the children to use a scaled unit size, such as a half or a fourth. Sometimes, they had difficulty using the appropriate unit size to measure both rods. For example, some students changed the size of a Dynamic Ruler's units while only considering how to equally partition one rod. However, Alex did more than partition only one rod at a time. He required his solution to equally partition both rods at the same time.

Generalized Commeasuring Type. Alex used similar strategies for the next task, Task 3-4. What made Task 3-4 different than its predecessor was that the children found multiple unit sizes to partition both rods, rather than a single unit size. The next episode shows how Alex solved Task 3-4 (Figure 53). 


\section{Figure 53}

Alex's Measurement With Multiple Partial Units
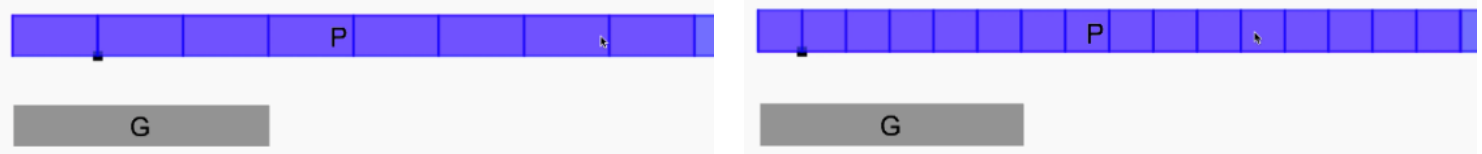

Note. The pink rod overlapped the Blue Dynamic Ruler, which is why the letter P appears in horizontal alignment with the ruler.

Alex: How many gray rods are needed to fit into the pink rod? Okay, so let's not - um, one [blue] section doesn't line up on the edge. Two sections doesn't work. Three, that's when it first works. So that's one, two, three, four, five, six, seven, eight. Um, so that means that there would be eight sections and since, um, three blue sections are equal to one gray section, there will be ... one gray, two grays - two grays and then two thirds of a gray. So, two and two thirds. And if you do this, um, you could do this like, um, with sixths, since it's like double. You can do that same thing I was talking about earlier, how you can double the number of or double the amount since there are, um, eight [blue sections that fit in the pink rod], it would be 16 if it was, like, split up into sixths. Um, so if we were to do that just to, like, see if that would work. Yeah. 16 sixths. So if we, if we move that up, it goes one, two, three, four, five, six, seven, eight, nine, 10, 11, 12, $13,14,15,16$. So, um, that, uh, is like an easier, uh, that's how you can do that. Find that without having to count it. (Alex, 596-607) 
Similarly, Alex commeasured two rods with the Blue Dynamic Ruler. He found a third size could measure both rods at the same time ("Three, that's when it first works.") and got the measurement result of $8 / 3$ (Figure 53-left). Following this solution, he anticipated that he could use sixths as double the thirds. He also found the new numerator (16) by doubling the original numerator (eight). By adjusting the size of the Blue Dynamic Ruler, he proved his conjecture was correct (Figure 53-right; "If we move that up, it goes one, two, three, four, five, six, seven, eight, nine, 10, 11, 12, 13, 14, 15, 16.”).

In the fraction-as-measurement tasks, the students could find several mathematical invariances such as equivalence. In order to understand the equivalent relation between quantities, it would be helpful for children to figure out quantitative invariance across continuous variations. The core idea of equivalent fractions is that any fraction has infinite forms to represent the same value (Empson \& Levi, 2011). Alex seemed to notice that even though he could partitioned the adjusted unit size into many equal-sized segments, the current form of the fraction was invariant ("how you can double the number of or double the amount").

\section{Charim's Case: Across Task Series 2 and 3 (2-4 to 3-1)}

Charim, a fourth grader, was chosen as the case who showed changed strategy types from the Determining to the Commeasuring across different Task Series (Tasks 2-4 and 3-1). The main difference between the tasks was the types of fractions. The fractions involved in Task 2-4 was $1 / 3$ (unit fraction) and in Task $3-1$ was $3 / 2$ (non-unit fraction). This change would be significant because it might be necessary to consider the relation between units and quantities due to the longer size of the leftover in the latter task. Charim used intermediate units in Tasks 2-4, but she changed her strategy by identifying 
partial unit sizes to measure in Task 3-1. In Task 2-4 (Figure 54-top), Charim measured by making the Green Dynamic Ruler's pieces the same size as the orange rod and found the size of the leftover on the brown rod with the Blue Dynamic Ruler (Determining Type). However, in Task 3-1 (Figure 54-bottom), even though she seemed to use two Dynamic Rulers, she was able to measure the pink rod with the Purple Dynamic Ruler, recognizing the ruler's units as half the size of the gray rod (Commeasuring Type).

\section{Figure 54}

Charim's Change in Strategy Types

Task 2-4

Q. How many Orange rods are needed to fit into the Brown rod?
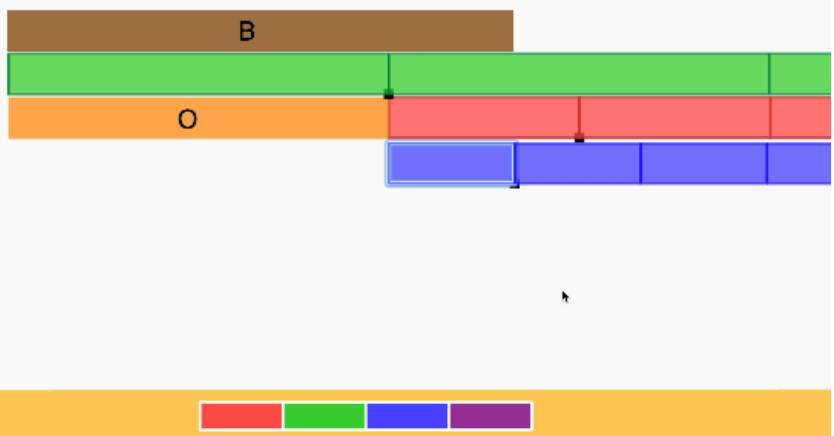

Task 3-1

Q. How many Gray rods are needed to fit into the Pink rod?

$\mathrm{P}$

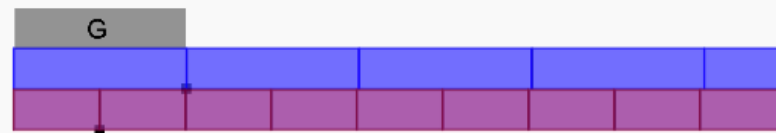


Determining Type. In the following episode, Charim solved Task 2-4 by using three different Dynamic Rulers (Figure 55).

\section{Figure 55}

Charim's Measurement With Green and Blue Dynamic Rulers
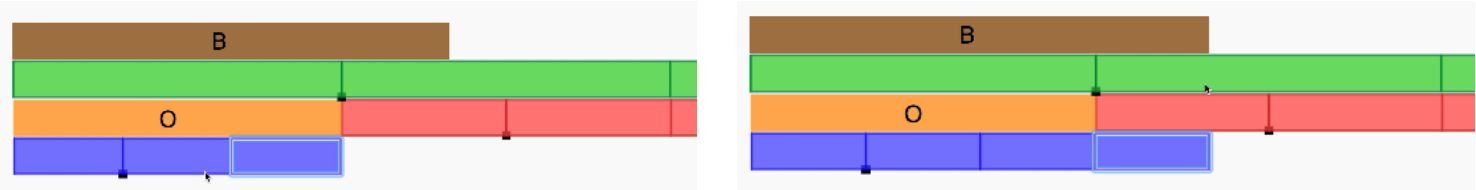

Charim: One and one fifth.

Y: One and one fifth of what?

Charim: Of blue.

Y: One and one fifth of blue rods are needed?

Charim: One orange and one blue.

Y: One orange and one blue. Okay. If you are using only orange rods, how can you tell me?

Charim: Only use orange rods? I can't make a brown rod because one is less than brown; if you have two, it is more than brown. So, you can't.

Y: Then, can you express this one box of blue in terms of orange?

Charim: Like five of them, no wait. It is one third.

Y: One-

Charim: One and one third. 
Y: One and one third of what?

Charim: One and one third - One and one third of three blues are the answer.

Y: Can you tell me one more time?

Charim: Like, one orange and three blues. No. One orange and one blue is the brown. And three blues make one orange. If you add one more, it will be exactly the same amount of the brown. So, it has to be four one thirds.

Y: Okay. My question is how did you decide three parts related to the orange?

Charim: Because here, if you move to here, I did orange, and I broke orange into halves. And I tried to break orange into halves, but it doesn't work. So, I moved a little. So, I got three, and the same amount as the one orange. (Charim, 160-174)

At first, Charim measured the leftover with one blue piece (Figure 55-left), but it was not clear why she expressed the leftover as a fifth. She was also confused about whether the orange rod or the Blue Dynamic Ruler was the referent unit. After a while, she stated the answer with two units, "One orange and one blue." When asked to explain a relationship between the two units, she moved the Blue Dynamic Ruler under the orange rod and realized that only three blue pieces equaled the orange rod (Figure 55left). She corrected her answer: "One and one third of three blues." After she related the blue piece to the orange rod ("three blues make one orange"), she added one more blue piece to match with the length of the brown rod ("four thirds"; Figure 55-right). She made a third size through trial and error, starting from a half size. Because the half size 
did not work to measure the leftover length of the brown rod, she decided to make a much smaller partial unit, and that was the size that equaled a third of the orange rod.

Charim initially implemented additive reasoning with two unit sizes: the orange rod (one green piece) and one blue piece. Without considering the relationship between two lengths, she counted two units separately. However, when asked the relationship between the two units, she developed and demonstrated her understanding of partial units based on the multiplicative structure of $4 / 3$ : one orange and one blue $\rightarrow$ one orange and one third of three blues $\rightarrow$ (one orange and one third of the orange rod $\rightarrow$ ) $4 / 3$ of the orange rod.

Commeasuring Type. Charim's progress toward multiplicative reasoning could be the foundation she used to solve Task 3-1, as demonstrated in the following episode.

\section{Figure 56}

Charim's Measurement With Green and Blue Dynamic Rulers
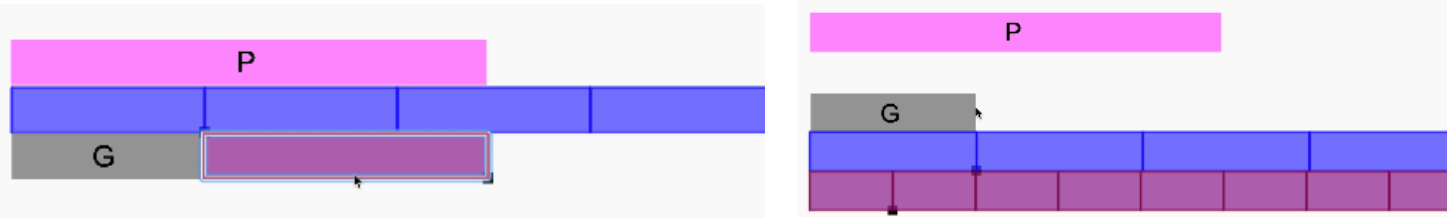

Charim: This is two and a half.

Y: How did you get that?

Charim: Because the two gray make almost pink, but, like, little pieces are left. When I do with purple, I press them exactly and [break] them into half. And one half is needed to fill in pink. And two grays are still there, and one more purple makes me to do pink. 
Y: It sounds great. And you mean — you mention about the half. Half of what?

Charim: Half of purple. Half of blue.

Y: Okay. Half of blue is?

Charim: Half. Half of blue is the half of gray.

$\mathrm{Y}:$ It is what?

Charim: It is ... into pink or gray?

Y: I mean, what you mention, the purple is half, why is it half?

Charim: Because when I do purple, this blue is the same thing as gray. And exactly half of blue and gray. Blue and gray are same amount of fractions. And it is the half of them. So, I thought it is the half.

Y: What about if we're only using the purple, then how many halves do you need?

Charim: Hmm. Five purple to fill like pink. (Charim, 187-199)

Charim first matched the Blue Dynamic Ruler's pieces with the length of the gray rods, placing the Blue Dynamic Ruler in the middle, between the two rods. With the Purple Dynamic Ruler, she tried to measure the difference between the pink and the gray rods (Figure 56-left). Because she could not find a meaningful result with this exploration, she changed how she used the Purple Dynamic Ruler. Shrinking the size of a purple piece so that it equaled half of the gray rod ("I press them exactly and [break] them into half'), she stacked two Dynamic Rulers under the gray rod (Figure 56-right). She could then also measure the pink rod with the Purple Dynamic Ruler: "Five purple to 
fill like pink". She found the rational relationship between the gray rod and the purple piece based on her manipulation of Dynamic Rulers. This strategy between quantities also seemed to help her make sense of the multiplicative structure of the improper fraction (five halves $=$ five copies of one half).

Compared to other task series, it took a while to investigate the relationship Charim perceived between the two rods with Dynamic Rulers. Because the size of the leftover in Task Series $2(1 / n)$ was different from Task Series $3(\mathrm{~m} / \mathrm{n})$, Charim might have spent more time exploring the Dynamic Ruler to figure out the most appropriate size of the unit. This longer exploration might have stemmed from the design difference between Task 2 and 3. After her exploration with various unit sizes, Charim concluded that she should use the half size of the gray rod as the new single scaled unit. 


\section{CHAPTER 5: DISCUSSION}

I examined children's understanding of fraction concepts from a measurement approach as the children in this study developed such conceptions within a digital technological environment. My analysis resulted in the identification of four types of strategy with a digital tool (Not Attending, Estimating, Determining, and Commeasuring). Even though children used similar strategy types, the way they dealt with the unit varied based on their understanding of fractions, length measurement, and technological features. When measuring the given length with the given unit size, some children paid little attention to the leftover component and struggled to quantify the relationship between the leftover length and the original unit (Not Attending). Others estimated the leftover using simple fractions (Estimating). Several students measured the whole-number part of the given length using one ruler and then measured the given length's leftover section using an additional ruler as an intermediate unit (Determining). At the Commeasuring Type, students generated dilated size units by adjusting the Dynamic Rulers. Then they further coordinated the given unit as well as the given linear quantity using this new unit size.

Because the use of technology was at the heart of solving measurement tasks, I also analyzed what patterns of children's strategies emerged depending on the characteristics of the fraction-as-measurement tasks. During the task-based interviews, what might influence these patterns in children's strategy types related to fraction understanding? To investigate this question, I delved into how children interacted with the Dynamic Ruler tool, using the instrumented mediation framework. With relation to instrumental genesis, a dynamic and ongoing process existed between the children and 
the Dynamic Rulers environment. The technological tool shaped children's understanding of fraction conceptions; their understanding also guided their use of the tool. Depending on task types and technological features, children articulated different conceptions related to unitization, measurement, and unit coordination. Regarding the process of semiotic mediation, signs (e.g., words) as symbolic tools were generated by the use of technological instruments and used in meaning making by internalizing mathematical activities with a tool. It was evident that children's fraction knowledge and tool proficiency influenced how they meaningfully used the tool. Different tasks types, technological features, and questions elicited different conceptions related to unitization, measurement, and unit coordination. Therefore, individual children might have different types of an instrument and develop different mathematical meaning based on such interactions with a tool. In this chapter, I first identify potential mechanisms that explain how different tasks elicit different types of strategy from the instrumented mediation perspective, then describe the implication and significance of these findings. I also discuss the potential of the strategy types framework. Finally, I provide suggestions for future research.

\section{Instrumented Mediation}

Through both the instrumental genesis and semiotic mediation perspectives, I analyzed how children could foster their access to mathematical meaning and develop various types of strategy in measurement tasks by using technology. Specifically, I investigated children's use of the Dynamic Ruler tool to conceptualize the meaning of fraction as measure within a tasks as well as across the tasks. In Figure 57, I return to the model first presented in Figure 2 and further articulate how the instrumented mediation 
framework - which merged the two distinct theories of instrumental genesis and semiotic mediation - was useful in understanding the process of meaning making of fractions using the dynamic tool. In the following section, I discuss how to apply this framework from a single situation (actions with knowledge-meaning making, affordances and constraints-meaning making) to a series of situations in problem solving (iterative instrumented mediation). Some components (e.g., dynamic features) of the instrumental perspective can be related to the process of mathematical meaning making in a single solution strategy, whereas other components (e.g., ramp-up in difficulty) can be explained across a series of the tasks.

\section{Figure 57}

Instrumented Mediation Process of Conceptualizing Fraction as Measure with a Digital Tool

\section{Instrumental genesis}

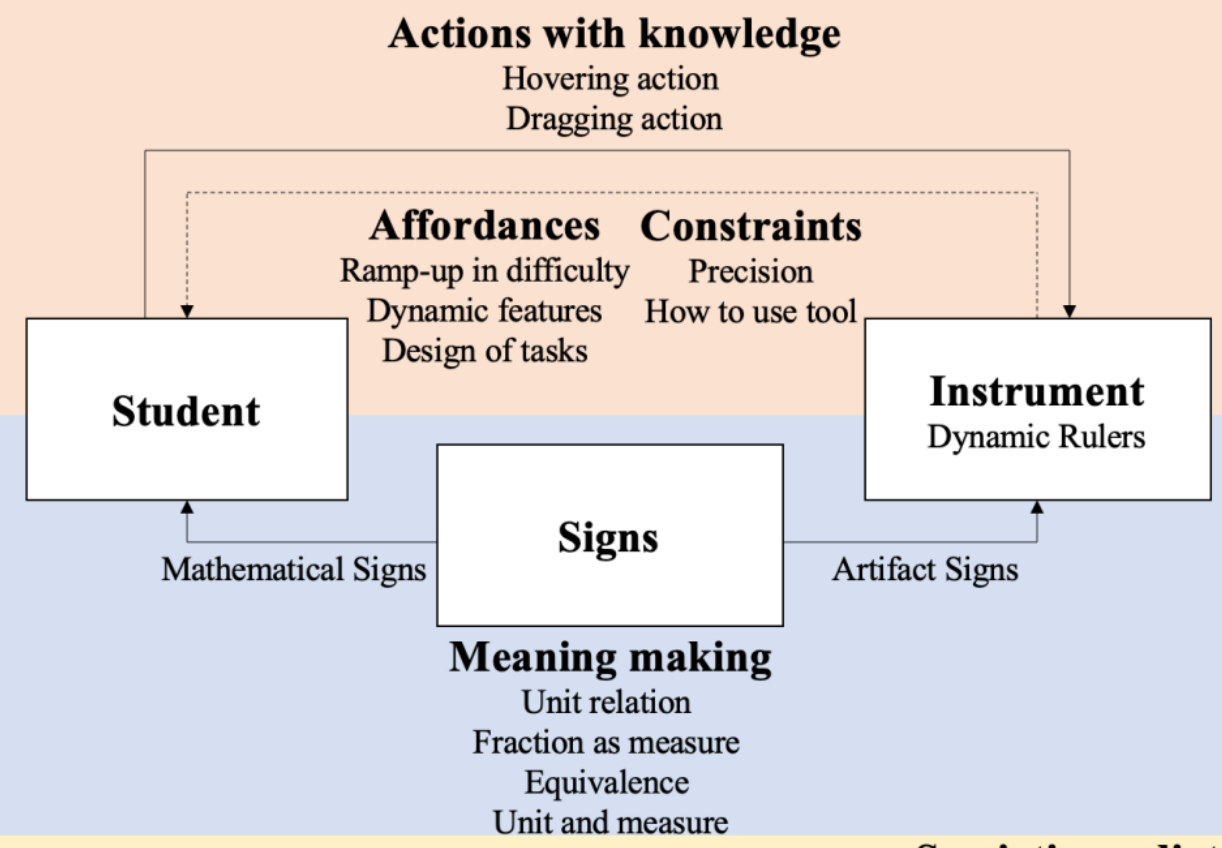

Semiotic mediation 


\section{Actions with Knowledge - Meaning Making}

With regard to the instrumentalization process (user's knowledge shapes the use of instrument), the children in this study could employ the hovering and the dragging actions for their purpose. Drawing their previous knowledge, children manipulate the tool for specific ways and ultimately established mathematical meaning.

During this study's Tutorial Task, the children first explored four functions in the tool (moving, selecting, adjusting, selecting). Employing these features, they could articulate and support their understanding of unit relation through mathematical actions. As one of significant actions, the children could construct intermediate units through changing the size of the Dynamic Ruler to make connections between original whole units and new partial units. Even though the children could make a Dynamic Ruler with pieces that were the same size as the given unit, some of them investigated the rational relationship between the given unit and a new partial unit by using other Dynamic Rulers to generate the new partial unit based on their previous knowledge.

For example, when solving Task 2-4, Sewoong initially constructed a Green Dynamic Ruler with pieces that were the same size as the given unit. Then, he brought up the Red Dynamic Ruler and the Blue Dynamic Ruler to figure out the leftover size. Matching one green segment and four red segments, he found that the leftover size must be at least one quarter of the original unit. Subsequently, he adjusted the Blue Dynamic Ruler to partition one red piece into four blue pieces. In this task, Sewoong manipulated three rulers and adjusted the size of the rulers differently. Finally, he figured out the relationship between one green segment (1), one red segment $(1 / 4)$, and one blue segment 
(1/16 ) with recursive measuring the leftovers, "Four quarters and a quarter of a quarter and the quarter".

What knowledge would influence such changing the size of the rulers with the dragging action? Because Sewoong was a third grader, he might have been more familiar with everyday fraction terms, such as a half and a quarter, rather than a third or a fourth as formal expressions in school mathematics. After the interview, he mentioned where his simple fraction expressions came from, "So, I saw basically money is one. Um, money is math too. And I used quarters, whole, and stuff like that, which is math too". This analogy might be why he only partitioned units into two or four parts as a repeated halving strategy. In Task 2-4, he volunteered to articulate his thinking process through partitioning the given unit into a specific number of parts ("Wait, what is? I need to check. I need to check the quarter. Hmm? Did I even measure it right?"). He measured the leftover rather than estimating it, even though the size of leftover was not familiar to him (one third), which is a key difference between the Estimating type and the Determining type. In a series of actions, he seemed to use his knowledge of partitioning money to the measuring situation. Like money unit (e.g., dollar), one length unit could be participated into two parts $(1 / 2)$ and the half of the unit also could be partitioned into two parts $(1 / 4)$. This might even provide a chance for children to enhance their strategies concerning a composite unit as unit relation, which refers to a unit of a unit ("a quarter of a quarter"). Such idea would not come up with simple estimating.

\section{Affordances and Constraints - Meaning Making}

Instrumental genesis is a theory concerning the transformation of an artifact to an instrument through the artifact's reciprocal interactions with its users. Via the process of 
instrumentation (i.e., instrument shapes cognition), the establishment of students' mathematical meaning was influenced by the affordances of the tool (e.g., the tool's dynamic features, an increase in cognitive complexity, the design of the measurement tasks) and the tool's constraints (e.g., precision, the children's comprehension of how to use tool).

The dynamic features of the Dynamic Ruler tool afforded opportunities for children to use their current understanding in specific ways. In particular, the ability to change the size of the Dynamic Ruler (continuously) supported some students in utilizing a commeasuring strategy that revealed multilevel composite unit conceptions about fractions and measurement. For example, Emily explored the Dynamic Ruler tool to look for an appropriate unit size to measure both of the given rods at the same time. This dynamic feature she employed to adjust the ruler's pieces might have provided a method to figure out whether a specific size of unit worked by continuous variations. This exploration might invite her to extend her understanding to find other equivalent fractions without actual manipulations of the tool, then she was able to test the conjecture through the use of the Dynamic Ruler.

On the other hand, as a constraint, some students had struggled with precise manipulation of the Dynamic Rulers. Due to the generic feature of the measuring tool, it might be not easy to make a precise length when adjusting the size of the ruler. Because children tended to interpret and reflect on what they did on the basis of visual representations, the way to manipulate the tools is critical to develop mathematical understandings of fraction relationships (Empson \& Levi, 2011). For example, in Task 31, Fran used the Blue Dynamic Ruler to make units of the same length as the gray rod, 
and she used the Purple Dynamic Ruler to represent half-size pieces of the gray rod. But two purple pieces were actually longer than one blue piece. In other words, a purple piece did not equal a half of the gray rod. In Task 3-2, when asked to use different unit sizes, she moved the black dot of the Blue Dynamic Ruler to the left to "make two halve of this." But she did not precisely partition the gray rod into two pieces of the Blue Dynamic Ruler. As a result, she could not achieve a quarter, which should have been half the size of a blue piece ("That's a half. So, then it's like a little bit more."). Therefore, in this case, Fran's limited understanding of the equal-partition concept in measurement might have caused her to make little imprecise manipulations in length measurement, much like Teo did in his case that was described previously. These imprecisions might have prompted the children to estimate the leftover part rather than use the Dynamic Ruler tool to verify the unit relations. These findings are perhaps related to intermediate conceptions - that is, the target conception was still forming for these children. Similar limitation was founded in the use of fraction strips (Brinker, 1997). When solving a fraction addition task $(2 / 5+2 / 8)$, fifth graders, Jay and Rachel, used fraction strips by lining them up 'end-to-end' to find the sum. Both students thought $5 / 8$ was a correct answer for the task and the manipulatives were used correctly. Even though this estimation would be a pretty close answer, a discrepancy arose by comparing to the actual sum $(26 / 40)$. Since the difference between $2 / 5$ and $5 / 8$ is $1 / 40$, students might pay little attention to the discrepancy in the context of using fraction strips. However, this small difference makes totally a different answer $(5 / 8$ vs $26 / 40)$.

One potential solution to prevent children's imprecise manipulations would be to add a snapping feature to the Dynamic Ruler tool for future studies. When moving a 
black dot to change the size of a Dynamic Ruler's segments, the snapping feature could assist in moving the ruler tool to a precise location that is related to other rods by matching a specific number of pieces to the rods. Still, children could adjust the size of the Dynamic Ruler's pieces continuously and at the same time they would make the sizes precisely as one, a half, a third, a fourth, and so on of the given unit (e.g., the gray rod).

\section{Iterative Instrument Mediation}

Over the course of completing this study's tasks, the children used a measurement approach that interacted with their fraction knowledge and strategies through the use of signs, such fraction words. The tool helped students to conceptualize fraction as measure and then understand the components of the concept over time: unit relation, equivalence, and the relationship between units and measure. The instrumented mediation can be applied to a single problem solution to articulate how a child conceptualizes mathematical meaning. However, it is also possible to apply to the series of problem solutions as an iterative process.

The tasks began with a focus on the relationship between two rods, where the given length could be measured with whole unit iterations of the given unit. However, as the students progressed through the tasks, many of them decided to use partial unit sizes to quantify the given length in terms of the given unit. The process of semiotic mediation stimulated this shift. The artifact signs and mathematical signs children generated illustrated central features of fraction as measure, including how a new partial unit relates to the original unit, how a new partial unit can measure the given unit and quantity, and how a given unit can be partitioned in many ways. 
For example, in Task 2-1, Alex constructed a Dynamic Ruler with pieces that were the same size as the given unit, then recognized that the leftover was a half by comparing it to the whole unit size ("So that's, it fills up one whole. And then if we look at that, that is one half."). During this task, even though Alex found the ratio between the leftover and the original unit, he only focused on providing a relative size for the leftover in terms of the unit size. In Task 3-4, he similarly constructed a ruler with pieces that were the same size as the given unit. However, Alex did not stop manipulating the Dynamic Ruler at that point (“one [blue] section doesn't line up on the edge"). He decided to keep investigating to find the ruler's appropriate unit size. He tried to make a ruler with pieces that were half the size of the given unit and then a third of the size ("Two sections doesn't work. Three, that's when it first works"). He concluded that the third was the appropriate unit for measuring the given length ("There would be eight sections and since, um, three blue sections are equal to one gray section ... two grays and then two thirds of a gray. "). During this strategy, Alex's measurement was not static, but evolved. He did not adjust the Dynamic Rulers just once but used continuous dynamic actions to find an appropriate unit size to measure the given length.

In the fraction-as-measurement task, because the ratios were selected specifically to increase difficulties across the progression of tasks, the children might have been stimulated to extend, modify, and generalize their strategies over the course of completing the tasks. Specifically, as an affordance, the design of the fraction-asmeasurement tasks situated children as they figured out fractional values through measuring, rather than merely eyeballing the lengths. In Task Series 1, some children easily anticipated the measure without actually measuring with the Dynamic Ruler. 
However, they felt it was more important to use the ruler tool to figure out the leftover parts in Task Series 2 and 3, which was the main intention of the tasks' design. Task Series 2 was designed to introduce a partial unit size, which the children would determine by adjusting the size of a Dynamic Ruler's pieces (Estimating/Determining). When compared to the tasks in the second series, Task Series 3 had much longer leftovers than the given unit sizes. These scenarios situated children to measure a given unit and length with the same partial unit size (Determining/Commeasuring). Therefore, the sequence of task series, in some cases, elicited a set of different unit conceptions as children had to adapt their previous strategies to new affordances. For example, Charim made a Dynamic Ruler's units the same size as the rod designated as a measurement unit, then she measured a leftover part as a third of the unit size in Task 2-4. However, this measuring approach did not work for Charim's next task (3-1) because the size of the task's leftover length $\left(1 \frac{1 / 2}{2}\right)$ was not a single unit fraction. This challenge with a new ratio between the task's two rods (unit fraction to non-unit fraction) might have compelled her to use different strategies by making a half-sized unit with a Dynamic Ruler.

\section{Directions for Future Research}

Based on the study's findings and limitations, I can suggest potential directions for future research. First, a question that arises from this study relates to the application of the Dynamic Ruler in classroom settings. This study examined individual students and investigated their fraction understanding through clinical interviews. From the findings of this study, classroom teachers might anticipate a range of solution strategies across the strategy type and prompt children to try various sizes of units by listening to their peers. However, it might be difficult to imagine how to facilitate group discussions and how to 
coordinate their use of a dynamic tool. In classrooms, teachers organize various artifacts to facilitate class-wide instrumental genesis and ensure opportunities to develop each student's instrumental genesis in technology learning environments. This collective approach is called instrumental orchestration. I wonder how teachers can utilize the Dynamic Ruler tool in classroom situations and how discursive interactions between children impact fractional meaning making.

Another avenue for future research is to extend conceptions of fractions beyond the measurement approach to fractions. Fraction as measure is one of five conceptions of fractions. The others are the part-whole, quotient, ratio, and operator conceptions (Behr et al., 1992; Kieren, 1988). Fraction as measure can provide a strong foundation for children to understand the complex meanings of fractions and learn advanced mathematics content in terms of types of units. However, this approach cannot cover all fraction meanings. Therefore, further studies are called for to investigate how the use of the Dynamic Ruler tool might impact students' understanding of the other fraction conceptions, such as partwhole or operator. For example, it might be possible to compare the types of understanding in other fractions concepts with pre/post tests to figure the impact of this measurement approach. Furthermore, I recommend that future studies examine the effect of conceptualizing fraction as measure without the technological tool. Finally, this study focused on students in grades 3-5. Future studies should explore how the Dynamic Ruler can support mathematics lessons for higher grade levels (e.g., algebra, geometry).

With regards to the equitable perspective, technology has the potential to lighten the learning burden for ELLs, lessening the negative impacts on ELLs who are somewhat unfamiliar with the language and creating opportunities for extended mathematical 
understanding (Ganesh \& Middleton, 2006). Some ELLs in this study did not always use fraction words (e.g., they used terms such as "two doubles," "zero decimal five," and "one second"). However, their mathematical actions were not different than those of native English speakers. This is not a trivial finding. If educators and researchers better understood how ELLs operationalize the concept of fraction as measure using visual representations in a digital tool, they could define a starting point from which to design an improved curriculum that better supports ELLs as they learn the fraction domain. From a deficit angle, the ELLs' lack of appropriate mathematical terminology might be an obstacle to teaching fractions. However, it could be further investigated how ELL's tool-generated mathematical signs and expressions can contribute rich discourse to understand fractions conceptually in classroom settings. Furthermore, this might provide a counter-story that focuses on who is capable of doing mathematics (de Araujo et al., 2016).

In this study's strategy types framework, I identified that children's strategies fell along a continuum from reasoning about a single unit relation using estimation to reasoning about multiple unit relationships using dynamic actions. Future research should seek to answer the following questions: What is a hypothetical learning trajectory that employs a dynamic tool to enhance children's conceptualization of fraction as measure? More broadly, how do children achieve mathematical understanding when using technological tools? 


\section{CHAPTER 6: CONCLUSION}

In this study, I sought to answer the following two research questions: What are the different ways that children use the Dynamic Ruler tool in approaching each fractionas-measurement task? What patterns emerge in children's strategies across the series of the fraction-as-measurement tasks?

To answer these questions, I analyzed screencast videos from clinical interviews with individual elementary students and developed a framework to analyze children's use of the dynamic tool and fraction understandings through exploratory case studies.

The children produced new words, actions, and diagrams to solve the tasks via mathematical activities in the Dynamic Ruler environment. The environment included the software, the particular microworlds, and tasks that I designed. The Dynamic Ruler seemed to help the children pay attention to how a leftover part and a unit relate in measurement and fractions. They could see how the measurements and fractions changed in relation to the ratio between two units.

This study's findings can be compared to the responses of participants from previous studies that focused on similar aspects of fractions. Dougherty et al. (2004) developed the Measure Up curriculum, which was influenced by the Russian mathematics curriculum. The spirit of that curriculum was to provide a meaningful foundation for young students to prepare for complicated mathematics through a measurement approach. A body of studies provided evidence about the effectiveness of this approach (e.g., Dougherty, 2008; Venenciano \& Heck, 2016). It is clear that the studies provided new insights into how the measurement approach's physical and symbolic representations prepared students to later succeed in advanced mathematics, 
such as rational numbers and algebra. However, it was not clear how the students' use of such concrete tools (e.g., rulers, beakers) helped them structure theoretical ideas. In this current study, I used an instrumented mediation framework to explain how such concrete tools, even nondigital tools, could help develop mathematical meaning.

This study's results raise more fundamental questions: How did the children use fractional terms as solutions for their tasks? What mediated the children to think about the relationships between two different unit lengths? These questions might be potentially related to the tasks' prompts. To probe children's strategies related to fraction as measure, there were various ways that I could have situated them in the tasks. In the first pilot study, for example, I used a written prompt that asked, "Which rod is longer?" This prompt gave the children an opportunity to think about the comparison between two rods, after which it was possible to ask them how many unit lengths were needed to measure one rod with the other. The prompt was quite open and even might be indirect to mediate children's strategies about unit relations. Therefore, I revised the prompt for this dissertation's main study so that the children could concentrate on measuring scenarios with partial unit sizes. In the main study, for example, the written prompt for Task Series 2 was, "How many orange rods are needed to fit into the blue rod?" Such a prompt was intended to be interpreted so that the orange rod was perceived as a unit of measure while the blue rod was perceived as an object to be measured. And this direct prompt mediated the children's expressions of the leftover amounts with fractional numbers (e.g., 11/2) or words (e.g., one and a half). The prompt required the children to derive answers in terms of the orange rod (“How many orange rods are needed?"). 
This mediation from the prompt explains the use of mixed numbers. Some children could explain the equivalence between the mixed numbers and improper fractions, but they used mixed numbers in Task Series 2 and 3. They tended to coordinate whole parts with the orange rod first and then estimate (or measure) the leftover. What if the prompt had only asked to express the relationship between the rods with fraction numbers? Some children might have approached the tasks from the part-whole concept to figure out fractions rather than the measuring concept. For example, using the part-whole concept, a child might have explained that the yellow rod is three pieces and the blue rod is five pieces; therefore, the answer is 3 out of 5 . This type of answer was rare in this study.

In one of the tasks, I asked students how they could find the length of an object (a rod) with prepartitioned units. Similar tasks were used by Simon et al. (2018), who found that students could use prepartitioned units when forming an initial concept of fractions and could come to understand that a quantity is measured by iterating the unit, a partial unit, or a combination of both. However, more general measurement situations have another facet that evaluates the length with undecided units (i.e., the quantity is given, and its unit is not). Students could adjust the unit size of this study's Dynamic Ruler and had an opportunity to commeasure a quantity and a unit with various expressions. In Task 2-1, for instance, one student measured a quantity with partial units by matching both right-end lines at the same time. The student came up with a result of $3 / 2$ because he measured the leftover between the given quantity and unit as the new partial unit (one half). This result suggests that some of the tasks used in my study might help students deepen their fraction conceptions as they use the dynamic tool, especially when 
contrasted with prior studies that found students lacked this understanding with static representations.

In the fraction domain, JavaBars (Steffe \& Olive, 2010) has been a predominant tool to examine children's understanding of fraction concepts. However, the complexity of both its interface and manipulations can be obstacles to children intuitively using the tool. Due to the iterating and partitioning features of the tool, JavaBars seems to emphasize an iterative approach to constructing and deconstructing fractions (e.g., three quarters is three iterations of one quarter of the whole unit). In comparison, this study's Dynamic Ruler tool supported the flexible use and understanding of fractions through continuous variation as well as intuitive interactive features (e.g., dragging, adjusting the increment size). Like DGS tools, for example, children could directly change the size of the Dynamic Ruler's increments by dragging one point. Dynamic features such as this provided a rich opportunity for students to explore different equipartitions of the unit size. In the case of the study participant named Alex, the tool even allowed him to generalize fraction as measure concepts. He also found that he could generate infinite equivalent fractions if he knew the exact fraction value of the measure. If Alex had used physical fraction manipulatives or JavaBars instead of the Dynamic Ruler tool, this exploration of equivalence would be hard to experience. But the Dynamic Rulers allowed for continuous adjustment so that Alex and other children could easily comprehend the way to commeasure and the relationships between equivalent fractions. Moreover, the digital ruler tool aligned such dynamic manipulation with the real-world act of measuring rather than iterating while constructing fractions. 


\section{Limitations}

This study examined elementary students' fraction understanding using a dynamic tool. I chose several representative and interesting cases from the participants' experiences to provide a cross-sectional view of the study's findings. However, the first limitation is that the participants might not represent typical elementary students. Rather, their parents (or often the students themselves) volunteered to participate in this study. I confined the sampling to only those students in a single public school district to control other potential variables related to regional factors, which might influence the study results. The participants consisted of 13 third graders, eight fourth graders, and nine fifth graders. I actively recruited them from diverse races, genders, and English-language proficiencies. However, the sampling was still not as rigorous as it would have had background information on each participant.

The second limitation relates to the interviewing process. I used clinical interviews that involved only one child at a time. However, it was unclear how much the children already knew in the fraction and measurement domains before their interviews. In addition, the preparation time in the study's Tutorial Task might have been insufficient to help students fully understand all the features of the tool, which were crucial in solving the fraction-as-measurement tasks. Some students might have grasped the tool's features quickly during the Tutorial Task, whereas other students may have struggled to articulate or advance their fraction knowledge because they had less familiarity with the tool and would have benefitted from a longer tutorial.

The third limitation is the reliability of coding process. I was a single coder who assigned strategy types for each solution strategy children used. Even though I had 
developed and refined the different strategy types with clear criteria, the process of coding could be subjective and biased. Therefore, the reliability in coding can be enhanced by the creation and maintenance of an operative codebook with examples and non-examples of responses at each strategy type (Miles, Huberman, \& Saldaña, 2013). Furthermore, additional coders can bolster the strength of this reliability issue with discussion and resolution of discrepancies.

A final limitation concerns the data collection device. Young children might be more familiar with a tablet PC with a touchscreen than a laptop with a mouse. But I utilized a laptop for the task-based interviews. The laptop's mouse cursor made it evident to me, the researcher recording their actions, how the children manipulated digital objects. A touchscreen may have been an inferior data collection device in this regard. However, it was possible that the children who had little experience with a laptop may have faced technical issues (e.g., clicking and dragging) when solving fraction-asmeasurement tasks.

\section{Final Remarks}

To enhance mathematics education, I tested and refined theories of how students come to understand fraction concepts with the use of a dynamic technology. I also produced a theory-driven, empirically tested set of tasks accompanied by illustrations of students' problem-solving in a dynamic digital environment. The findings will help bridge the gap between theory and practice and analyze alternative methods for using fractions to teach number and math fundamentals, as well as advanced concepts such as algebra and geometry. This study aimed to use the fraction as measure concept instead of the part-whole concept to enhance students' knowledge. As a result, my findings offer 
one way to expand students' typical conceptions of fractions. By developing a robust understanding of fractions, this study's learning approach has the potential to increase students' critical thinking skills both within and beyond mathematics.

Furthermore, dynamic technology is an influential tool, but its potential has rarely reached a type where the technology is actively developing students' cognitive systems. I hope this study's findings will advance the fundamental idea that dynamic technology can and should support conceptual understanding in fraction instruction, mathematics more broadly, and perhaps all facets of classroom instruction. 


\section{REFERENCES}

Artigue, M. (2002). Learning mathematics in a CAS environment: The genesis of a reflection about instrumentation and the dialectics between technical and conceptual work. International Journal of Computers for Mathematical Learning, 7(3), 245-274.

Arzarello, F. \& Robutti, O. (2008). Framing the embodied mind approach within a multimodal paradigm, In Lyn English, M. Bartolini Bussi, G. Jones, R. Lesh e D. Tirosh (eds.), Handbook of International Research in Mathematics Education (LEA, USA), 2nd revised edition.

Barnett-Clarke, C., Fisher, W., Marks, R., \& Ross, S. (2010). Developing essential understanding of rational numbers for teaching mathematics in grades 3-5. Essential Understanding Series. Reston, VA: National Council of Teachers of Mathematics.

Bartolini Bussi, M. G. B., \& Baccaglini-Frank, A. (2015). Geometry in early years: sowing seeds for a mathematical definition of squares and rectangles. $Z D M, 47(3)$, 391-405.

Bartolini Bussi, M. G., \& Mariotti, M. A. (2008). Semiotic mediation in the mathematics classroom: artefacts and signs after a Vygotskian perspective. In: L. English, M. Bartolini Bussi, G. Jones, R. Lesh, \& D. Tirosh (Eds.), Handbook of international research in mathematics education (pp. 720-749, 2nd ed.). Mahwah: Erlbaum

Battista, M. T. (2008). Representations and cognitive objects in modern school geometry. Research on technology and the teaching and learning of mathematics: Cases and perspectives, 2, 341-362.

Battista, M. T. (2012). Cognition-based Assessment and Teaching of Place Value: Building on Students' Reasoning. Portsmouth, NH: Heinemann. 
Behr, M.J., Harel, G., Post, Th. R., \& Lesh, R. (1992). Rational number, ratio, and proportion. In D. A. Grouws (Ed.), Handbook of Research on Mathematics Teaching and Learning (pp. 296-332). New York, NY: Macmilian Publishing Company.

Brenner, M. E., Herman, S., Ho, H. Z., \& Zimmer, J. M. (1999). Cross-national comparison of representational competence. Journal for Research in Mathematics Education, 30(5), 541-557.

Brinker, L. (1997). Using structured representations to solve fraction problems: A discussion of seven students 'strategies. Paper presented at the annual meeting of the American Educational Research Association, Chicago, IL.

Brousseau, G., Brousseau, N., \& Warfield, G. (2014). Teaching fractions through situations: A fundamental experiment. Dordrecht, Heidelberg, New York and London: Springer.

Carpenter, T. P., Fennema, E., Franke, M. L., Levi, L., \& Empson, S. B. (2014).

Children's mathematics: Cognitively guided instruction. Portsmouth, TX: Heinemann.

Charalambous, C. Y., \& Pitta-Pantazi, D. (2007). Drawing on a theoretical model to study students' understandings of fractions. Educational studies in mathematics, 64(3), 293-316.

Charmaz, K. (2014). Constructing grounded theory (2nd ed.). Thousand Oaks, CA: Sage Publications Ltd.

Chval, K. B., Lannin, J. K., \& Jones, D. (2013). Putting essential understanding of multiplication and division into practice in grades 3-5. Reston, VA: National Council of Teachers of Mathematics. 
Clarke, D. M., \& Roche, A. (2009). Students' fraction comparison strategies as a window into robust understanding and possible pointers for instruction. Educational Studies in Mathematics, 72(1), 127-138.

Clements, D. H., \& Stephan, M. (2004). Measurement in Pre-K to Grade 2 mathematics. In D. H. Clements, J. Sarama \& A. DiBiase (Eds.), Engaging young children in mathematics: Standards for early childhood mathematics education (pp. 299-317). Mahwah, NJ: Lawrence Erlbaum Associates.

Confrey, G. (1994). Splitting, similarity, and rate of change: A new approach to multiplication and exponential. In G. Harel \& G. Confrey (Eds.), The development of multiplicative reasoning in the learning of mathematics (pp. 291-330). Albany, NY: State University of New York Press.

Davydov, V. V., \& Tsvetkovich, Z. H. (1991). The object sources of the concept of fraction. In V. V. Davydov (Soviet Edition Editor) \& L. P. Steffe (English Language Editor), Soviet studies in mathematics education: Psychological abilities of primary school children in learning mathematics (pp. 86-147). Reston, VA: National Council of Teachers of Mathematics.

Davydov, V.V. (1988). The concept of theoretical generalization and problems of educational psychology. Studies in Soviet Thought, 36, 169-202.

de Araujo, Z., Smith, E., \& Sakow, M. (2016). Reflecting on the dialogue regarding the mathematics education of English learners. Journal of Urban Mathematics Education, 9(2), 33-48.

DeWolf, M., \& Vosniadou, S. (2015). The representation of fraction magnitudes and the whole number bias reconsidered. Learning and Instruction, 37, 39-49. 
Dick, T. P., \& Hollebrands, K. F. (2011). Focus in high school mathematics: Technology to support reasoning and sense making. Reston, VA: National Council of Teachers of Mathematics.

Dougherty, B. J. (2008). Measure up: A quantitative view of early algebra. In J. J. Kaput, D. W. Carraher, \& M. L. Blanton (Eds.), Algebra in the early grades (pp. 389-412). New York, NY: Lawrence Erlbaum Associates.

Dougherty, B. J., Fay Z., \& Claire O. (2005). Measure Up: Grade 1-5. Honolulu, HW: University of Hawaii Curriculum Research and Development Group.

Dougherty, B., \& Venenciano, L. (2007). Measure Up for understanding. Teaching Children Mathematics, 13(9), 452-456.

Drijvers, P., Godino, J. D., Font, V., \& Trouche, L. (2013). One episode, two lenses. Educational Studies in Mathematics, 82(1), 23-49.

Drijvers, P., \& Gravemeijer, K. (2005). Computer algebra as an instrument: Examples of algebraic schemes. In D. Guin, K. Ruthven, \& L. Trouche (Eds.), The didactical challenge of symbolic calculators (pp. 163-196). New York, NY: Springer.

Drijvers, P., Kieran, C., \& Mariotti, M. A. (2010b). Integrating technology into mathematics education: Theoretical perspectives. In C. Hoyles \& J.-B. Lagrange (Eds.), Mathematics education and technology: Rethinking the terrain (pp. 89-132). New York, NY: Springer.

Drijvers, P. \& Trouche, L. (2008). From artefacts to instruments: A theoretical framework behind the orchestra metaphor. In G. W. Blume \& M. K. Heid (Eds.), Research on technology and the teaching and learning of mathematics, Volume 2 (pp. 363-392). Charlotte, NC: Information Age. 
Empson, S. B. (1999). Equal sharing and shared meaning: The development of fraction concepts in a first-grade classroom. Cognition and Instruction, 17, 283-342.

Empson, S. B., \& Levi, L. (2011). Extending Children's Mathematics: Fractions and Decimals: [innovations in Cognitively Guided Instruction]. Portsmouth, NH: Heinemann.

Empson, S. B., Junk, D., Dominguez, H., \& Turner, E. (2006). Fractions as the coordination of multiplicatively related quantities: A cross-sectional study of children's thinking. Educational Studies in Mathematics, 63(1), 1-28.

Freiman, V., Polotskaia, E., \& Savard, A. (2017). Using a computer-based learning task to promote work on mathematical relationships in the context of word problems in early grades. ZDM, 49(6), 835-849.

Fuchs, L. S., Schumacher, R. F., Long, J., Namkung, J., Hamlett, C. L., Cirino, P. T.,... Changas, P. (2013). Improving at-risk learners' understanding of fractions. Journal of Educational Psychology, 105, 683-700.

Ganesh, T. G., \& Middleton, J. A. (2006). Challenges in linguistically and culturally diverse elementary settings with math instruction using learning technologies. The Urban Review, 38(2), 101-143.

Ginsburg, H. (1997). Entering the child's mind: The clinical interview in psychological research and practice. UK: Cambridge University Press.

Goldin, G. A. (1997). Chapter 4: Observing mathematical problem solving through taskbased interviews. Journal for Research in Mathematics Education. Monograph, 40177. 
Guest, G., Bunce, A., \& Johnson, L. (2006). How many interviews are enough? An experiment with data saturation and variability. Field Methods, 18(1), 59-82.

Hackenberg, A. J. (2010). Students' reasoning with reversible multiplicative relationships. Cognition and Instruction, 28(4), 383-432.

Hackenberg, A. J., \& Tillema, E. S. (2009). Students' whole number multiplicative concepts: A critical constructive resource for fraction composition schemes. The Journal of Mathematical Behavior, 28(1), 1-18.

Heid, M. K., \& Blume, G. W. (Eds.). (2008). Research on technology in the teaching and learning of mathematics: Research syntheses (Vol. 1). Charlotte, NC: Information Age Publishing.

Hollebrands, K. F. (2007). The role of a dynamic software program for geometry in the strategies high school mathematics students employ. Journal for research in mathematics education, 38(2), 164-192.

Hoyles, C., \& Noss, R. (2003). What can digital technologies take from and bring to research in mathematics education? In A.J. Bishop, M.A. Clements, C. Keitel, J. Kilpatrick \& F. Leung (Eds.), Second International Handbook of Mathematics Education, Vol 1, (pp. 323-349). Dordrecht: Kluwer Academic Publishers.

Hunting, R. P., Davis, G., \& Pearn, C. A. (1996). Engaging whole-number knowledge for rational-number learning using a computer-based tool. Journal for Research in Mathematics Education, 27(3), 354-379.

I, J. \& Dougherty, B., \& Berkaliev, Z. (2015). Unit matters. Teaching Children Mathematics, 22(3), 170-176. 
I, J., Martinez, R., \& Dougherty, B. (2018). Misconceptions on part-part-whole proportional relationships using proportional division problems. Journal of Investigations in Mathematics Learning, 12(2), 67-81.

Ifrah, G. (2001). The universal history of numbers: From prehistory to the invention of the computer. New York: John Wiley.

Jackiw, N., \& Sinclair, N. (2009). Sounds and pictures: Dynamism and dualism in dynamic geometry. ZDM, 41(4), 413-426.

Jahnke, I. (2016). Digital didactical designs: Teaching and learning in cross action spaces. New York, NY: Routledge.

Kamii, C., \& Clark, F. B. (1995). Equivalent fractions: Their difficulty and educational implications. The Journal of Mathematical Behavior, 14(4), 365-378.

Kaput, J. J. (1985). Multiplicative Word Problems and Intensive Quantities: An Integrated Software Response. Technical Report, 85-19.

Kaput, J. J. (1992). Technology and mathematics education. In D. A. Grouws, Handbook of teaching and learning mathematics (pp. 515-556). New York: Macmillan.

Karpov, Y. V., \& Bransford, J. D. (1995). LS Vygotsky and the doctrine of empirical and theoretical learning. Educational Psychologist, 30(2), 61-66.

Kaur, H. (2015). Two aspects of young children's thinking about different types of dynamic triangles: prototypicality and inclusion. ZDM, 47(3), 407-420.

Kieren, T.E. (1976). On the mathematical, cognitive, and instructional foundations of rational numbers. In R. Lesh (Ed.), Number and measurement (pp. 101-151). Columbus, OH: ERIC/SMEAC. 
Kieren, T. E. (1988). Personal knowledge of rational numbers: Its intuitive and formal development. In J. Hiebert \& M. J. Behr (Eds.), Number concepts and operations in the middle grades (pp. 162-181). Hillsdale, NJ: Erlba

Kieren, T. E. (1993). Rational and fractional numbers: From quotient fields to recursive understanding. In T. P. Carpenter, E. Fennema, \& T. A. Romberg (Eds.), Rational numbers: An integration of research (pp. 49 - 84). Hillsdale, NJ: Lawrence Erlbaum Associates, Inc.

Kieren, T. E. (1994). Multiple views of multiplicative reasoning. In G. Harel \& G. Confrey (Eds.), The development of multiplicative reasoning in the learning of mathematics (pp. 387-397). Albany, NY: State University of New York Press.

Konold, C., Harradine, A., \& Kazak, S. (2007). Understanding distributions by modeling them. International Journal of Computers for Mathematical Learning, 12(3), 217230.

Laborde, C. (1993). The computer as part of the learning environment: the case of geometry. In Learning from computers: Mathematics education and technology (pp. 48-67). Springer, Berlin, Heidelberg.

Laborde, J. M. (2016). Technology-enhanced teaching/learning at a new type with dynamic mathematics as implemented in the new Cabri. Digital curricula in school mathematics, 53-74.

Lamon, S. J. (1996). The development of unitizing: Its role in children's partitioning strategies. Journal for Research in Mathematics Education, 27(2), 170-193. 
Lamon, S. J. (2007). Rational numbers and proportional reasoning. In F. K. J. Lester (Ed.), Second handbook of research on mathematics teaching and learning (pp. 629667). Charlotte, NC: Information Age.

Lamon, S. J. (2012). Teaching fractions and ratios for understanding: Essential content knowledge and instructional strategies for teachers (3rd ed.). New York, NY: Routledge.

Mack, N. K. (2001). Building on informal knowledge through instruction in a complex content domain: Partitioning, units, and understanding multiplication of fractions. Journal for Research in Mathematics Education, 32(3), 267-295.

Maschietto, M. (2015). The arithmetical machine Zero+ 1 in mathematics laboratory: instrumental genesis and semiotic mediation. International Journal of Science and Mathematics Education, 13(1), 121-144.

Maschietto, M., \& Soury-Lavergne, S. (2013). Designing a duo of material and digital artifacts: the pascaline and Cabri Elem e-books in primary school mathematics. ZDM, 45(7), 959-971.

Miles, M. B., Huberman, A. M., \& Saldaña, J. (2014). Qualitative data analysis: A methods sourcebook. Thousand Oaks, CA: Sage.

Moreno-Armella, L., Hegedus, S. J., \& Kaput, J. J. (2008). From static to dynamic mathematics: Historical and representational perspectives. Educational Studies in Mathematics, 68(2), 99-111.

Moss, J. (2005). Pipes, tubes, and beakers: Teaching rational number. In J. Bransford and S. Donovan (Eds.), How Children Learn: History, Science and Mathematics in the Classroom, (pp.309-350). Washington, DC: National Academies Press. 
Morris, A. K. (2000). A teaching experiment: Introducing fourth graders to fractions from the viewpoint of measuring quantities using Davydov's mathematics curriculum. Focus on Learning Problems in Mathematics, 22 (2), 33-84.

National Council of Teachers of Mathematics. (2014). Principles to actions: Ensuring mathematical success for all. Reston, VA: Author.

National Mathematics Advisory Panel. (2008). Foundations for success: The final report of the National Mathematics Advisory Panel. Washington, DC: U.S. Department of Education.

National Research Council. (2001). Adding it up: Helping children learn mathematics. Washington, DC: National Academy Press.

Ng, O. L., \& Sinclair, N. (2015). Young children reasoning about symmetry in a dynamic geometry environment. ZDM, 47(3), 421-434.

Ni, Y. J. (2001). Semantic domains of rational numbers and the acquisition of fraction equivalence. Contemporary Educational Psychology, 26, 400-417.

Ni, Y., \& Zhou, Y. D. (2005). Teaching and learning fraction and rational numbers: The origins and implications of whole number bias. Educational Psychologist, 40(1), 27 52.

Olive, J., \& Lobato, J. (2008). The learning of rational number concepts using technology. In M. K. Heid \& G. W. Blume (Eds.), Research on technology and the teaching and learning of mathematics: Research syntheses (pp. 1-54). Charlotte, NC: Information Age and the National Council of Teachers of Mathematics. 
Olive, J., \& Vomvoridi, E. (2006). Making sense of instruction on fractions when a student lacks necessary fractional schemes: The case of Tim. Journal of Mathematical Behavior, 25, 18-45.

Piaget, J., Inhelder, B., \& Szeminska, A. (1960). The child's conception of geometry. New York, NY: Basic.

Pitkethly, A., \& Hunting, R. (1996). A review of recent research in the area of initial fraction concepts. Educational Studies in Mathematics, 30(1), 5-38.

Roschelle, J., Kaput, J., \& Stroup, W. (2000). SimCalc: Accelerating students' engagement with the mathematics of change. Innovations in science and mathematics education: Advanced designs for technologies of learning, 47-75.

Roschelle, J., Noss, R., Blikstein, P., \& Jackiw, N. (2017). Technology for learning mathematics. In J. Cai (Ed.), Compendium for Research in Mathematics Education. Reston, VA: National Council of Teachers of Mathematics. 273-296.

Saxe, G. B., Taylor, E. V., McIntosh, C., \& Gearhart, M. (2005). Representing fractions with standard notation: A developmental analysis. Journal for research in mathematics education, 36(2), 137-157.

Saldaña, J. (2015). The coding manual for qualitative researchers (3rd ed.). London, UK: Sage.

Schmidt, W. H., McKnight, C. C., \& Raizen, S. A. (1997). A splintered vision: An investigation of U.S. science and mathematics education. Hingham, MA: Kluwer.

Schmittau, J. (2005). The development of algebraic thinking. A Vygotskian perspective. ZDM, 37(1), 16-22. 
Schmittau, J., \& Morris, A. (2004). The development of algebra in the elementary mathematics curriculum of VV Davydov. The Mathematics Educator, 8(1), 60-87.

Schoenfeld, A. H. (2002). Making mathematics work for all children: Issues of standards, testing, and equity. Educational researcher, 31(1), 13-25.

Schwartz, J. L. (1988). Intensive quantity and referent transforming arithmetic operations. Research Agenda for Mathematics Education Number Concepts and Operations in the Middle Grades, 2, 41-52.

Siegler, R. S., \& Pyke, A. A. (2013). Developmental and individual differences in understanding of fractions. Developmental psychology, 49(10), 1994-2004.

Simon, M. A., Placa, N., \& Avitzur, A. (2016). Participatory and anticipatory stages of mathematical concept learning: Further empirical and theoretical development. Journal for Research in Mathematics Education, 47(1), 63-93.

Simon, M. A., Placa, N., Avitzur, A., \& Kara, M. (2018). Promoting a concept of fraction-as-measure: A study of the Learning Through Activity research program. The Journal of Mathematical Behavior, 52, 122-133.

Sinclair, N., \& Crespo, S. (2006). Learning mathematics in dynamic computer environments. Teaching Children Mathematics, 12(9), 436-444.

Soury-Lavergne, S., \& Maschietto, M. (2015). Articulation of spatial and geometrical knowledge in problem solving with technology at primary school. ZDM, 47(3), 435449.

Steffe, L. P. (1992). Schemes of action and operation involving composite units. Learning and Individual Differences, 4(3), 259-309. 
Steffe, L. P., \& Olive, J. (2002). Design and use of computer tools for interactive mathematical activity (TIMA). Journal of Educational Computing Research, 27(1), $55-76$.

Steffe, L. P., \& Olive, J. (2010). Children's fractional knowledge. New York: Springer. Streefland, L. (1991). Fractions in realistic mathematics education. Boston: Kluwer. Streefland, L. (1993). Fractions: A realistic approach. In T. P. Carpenter, E. Fennema, \& T. A. Romberg (Eds.), Rational numbers: An integration of research (pp. 289-325). Hillsdale, NJ: Lawrence Erlbaum Associates, Inc.

Suh, J., Moyer, P. S., \& Heo, H. J. (2005). Examining technology uses in the classroom: Developing fraction sense using virtual manipulative concept tutorials. Journal of Interactive Online Learning, 3(4), 1-21.

Taylan, R. D., \& da Ponte, J. P. (2016). Investigating pedagogical content knowledge-inaction. REDIMAT, 5(3), 212-234.

Tarr, J. E., Reys, R. E., Reys, B. J., Chavez, O., Shih, J., \& Osterlind, S. J. (2008). The impact of middle-grades mathematics curricula and the classroom learning environment on student achievement. Journal for Research in Mathematics Education, 39(3), 247-280.

Thompson, P. W., \& Saldanha, L. A. (2003). Fractions and multiplicative reasoning. In J. Kilpatrick, W. Gary Martin, \& D. Schifter (Eds.), A research companion to principles and standards for school mathematics (pp. 95-113). Reston, VA: The National Council of Teachers of Mathematics. 
Tirosh, D. (2000). Enhancing prospective teachers' knowledge of children's conceptions: The case of division of fractions. Journal for research in Mathematics Education, $31(1), 5-25$

Trouche, L. (2004). Managing the complexity of human/machine interactions in computerized learning environments: Guiding students' command process through instrumental orchestrations. International Journal of Computers for mathematical learning, 9(3), 281-307.

Tzur, R. (1999). An integrated study of children's construction of improper fractions and the teacher's role in promoting that learning. Journal for Research in Mathematics Education, 30, 390-416.

Tzur, R., \& Simon, M. (2004). Distinguishing two stages of mathematics conceptual learning. International Journal of Science and Mathematics Education, 2(2), $287-$ 304.

Vergnaud, G. (1994). Multiplicative conceptual field: What and why? In G. Harel \& J. Confrey (Eds.), The development of multiplicative reasoning in the learning of mathematics (pp. 41-59). Albany, NY: State University of New York Press.

Verillon, P., \& Rabardel, P. (1995). Cognition and artifacts: A contribution to the study of though in relation to instrumented activity. European journal of psychology of education, 10(1), 77-101.

Vygotsky, L. S. (1978). Mind in society. Cambridge, MA: Harvard University Press Vygotsky, L. S. (1981). The instrumental method in psychology. The concept of activity in Soviet psychology, 137, 143. 
Vygotsky, L. S. (1987). Thinking and speech. In R.W. Rieber \& A.S. Carton (Eds.), The collected works of L.S. Vygotsky, Volume 1: Problems of general psychology (pp. 39285). New York: Plenum Press. (Original work published 1934.)

Vygotsky, L. S. (1997). The collected works of LS Vygotsky: Problems of the theory and history of psychology (Vol. 3). Springer Science \& Business Media.

Webel, C., \& DeLeeuw, W. W. (2016). Meaning for fraction multiplication: Thematic analysis of mathematical talk in three fifth grade classes. The Journal of Mathematical Behavior, 41, 123-140.

Webel, C., Krupa, E., \& McManus, J. (2016). Using representations of fraction multiplication. Teaching Children Mathematics, 22(6), 366-373.

Webel, C., \& Otten, S. (2015). Teaching in a world with Photomath. The Mathematics Teachers, 109(5), 368-373.

Yeo, S. (2020, April 1-4). (Re)Thinking fractions with dynamic ruler [Conference session]. The centennial annual meeting of National Council of Teachers of Mathematics. Chicago, IL. (Conference canceled)

Yin, R. K. (2014). Case study research: Design and methods (5th ed.). Thousand Oaks, CA: Sage.

Young, J., Gorumek, F., \& Hamilton, C. (2018). Technology effectiveness in the mathematics classroom: a systematic review of meta-analytic research. Journal of Computers in Education, 5(2), 133-148. 


\section{Appendix A. Interview protocol}

1. Introduction script (The purpose here is to introduce the purpose and expectations, and to establish rapport with the child. This can be flexible, but be clear that you are interested in how the child THINKS about the tasks more than how correct (s)he is.

- What grade are you in? How old are you?

- How do you like your math class?

- I'm interested in how children solve problems by using technology. I'd like to ask you to solve a few problems and I'll also ask you questions about what you are thinking. By doing this, I might be able to help other students learn to solve such problems better. Of course, what you do here with me won't affect your grades in any way. Actually, I'm interested in how you are thinking about the problems and not whether or not you get them correct. It's ok to change your mind, it's ok if you get stuck, and you can ask questions anytime you want.

\section{General Protocol Questions}

- (After reading the prompt on the screen to a child) Do you have anything to ask about this problem?

- Ask clarifying questions about the student's strategy:

- Tell me about your strategy.

- How did you decide to...?

- What does (part of the picture, etc.) represent?

- Ask extending questions about the strategy:

- Can you solve this problem different way?

- Can you express your idea with a form of number sentence?

- At the end of the sessions, if the student has NOT used a unit adjustment: Can you try to solve this task again by using Dynamic Ruler? 


\section{Measurement as Fraction tasks}

Tutorial Task: The use of Dynamic Ruler: moving, adjusting, trimming, and pulling up new ruler

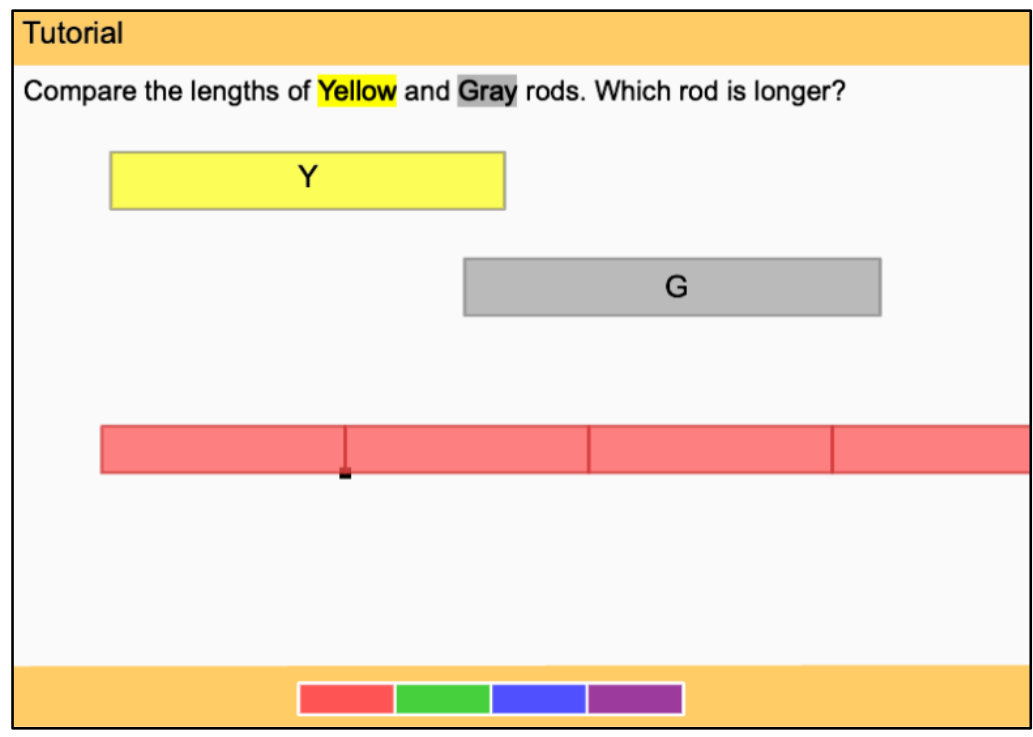

a. [moving] Can you move around the red ruler?

b. [adjusting] What does the black point do on the dynamic ruler?

c. [trimming] What happens if you click the second rod?

d. [pulling up] What happens if you click the bottom green? 


\section{Task Series 1: Using units to measure a length}

[task order: $1 \rightarrow 2(\rightarrow$ optional 3$) \rightarrow 4$ ]

\begin{tabular}{|l|l|l|l|l|}
\hline Task 1-1 & \multicolumn{2}{|l|}{ Task 1-2 } & \\
\hline
\end{tabular}

a. How did you get the number of Yellow rods?

b. (if a child mentioned the number only (e.g., four)) Four of what?

c. One red bar and yellow are the same length? How do you know that?

d. (when adjusted the unit) How did you decide the size of that unit?

e. (If a child did not use Dynamic Ruler) Can you use Dynamic Ruler to solve the task?

f. (1-1) What if you use the Dynamic Ruler twice longer than Yellow rod? How the number can be changed? Why is the number getting smaller?

g. how much of the blue rod fits into the yellow rod?

h. (1-3) How did you choose the red (or green) Dynamic Ruler?

i. (1-3) Both red and green Dynamic Ruler has the same answer? Why? 
Task Series 2: Using partial units to measure a length (below 2)

[task order: $1 \rightarrow 2(\rightarrow$ optional 3$) \rightarrow 4$ ]

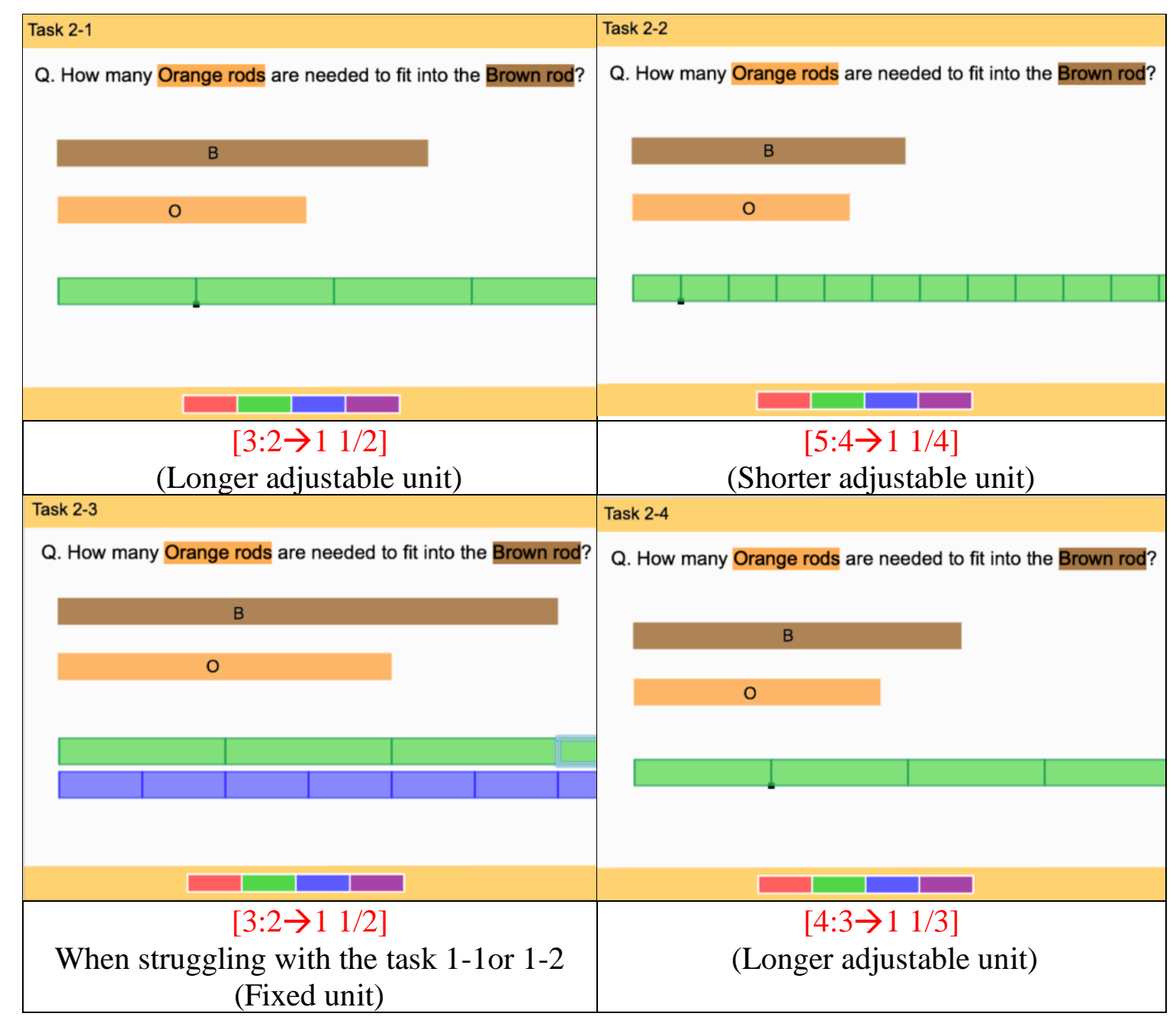

a. How did you get the number of Orange rods?

b. (if a child mentioned the number only (e.g., one and a half)) One and a half of what? half of what?

c. (if a child stated the bigger number than the answer (e.g., two and a half)) Can you show me how two and a half of Orange rods fit into Brown rod?

d. (when adjusted the unit) How did you decide the size of that unit?

e. Why did you put Dynamic Ruler over that place? Is there another way to use the ruler?

f. (If a child did not use Dynamic Ruler) Can you use Dynamic Ruler to solve the task?

g. (Task 2-3) How did you choose the green (or blue) Dynamic Ruler? 


\section{Task Series 3: Using partial units to measure a length (over 2)}

\section{[task order: $1 \rightarrow 2(\rightarrow$ optional 3$) \rightarrow 4$ ]}

\begin{tabular}{|c|c|}
\hline \multirow{2}{*}{$\begin{array}{l}\text { Task 3-1 } \\
\text { Q. How many Gray rods are needed to fit into the Pink rod? }\end{array}$} & \multirow{2}{*}{$\begin{array}{l}\text { Task 3-2 } \\
\text { Q. How many Gray rods are needed to fit into the Pink rod? }\end{array}$} \\
\hline & \\
\hline $\mathrm{P}$ & $\mathrm{P}$ \\
\hline G & G \\
\hline \begin{tabular}{|l|l|l|l|} 
& & & \\
\end{tabular} & 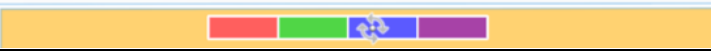 \\
\hline $\begin{array}{c}{[5: 2 \rightarrow 21 / 2]} \\
\text { (Longer adjustable unit) }\end{array}$ & $\begin{array}{c}{[11: 4 \rightarrow 23 / 4]} \\
\text { (Shorter adjustable unit) }\end{array}$ \\
\hline Task 3-3 & Task 3-4 \\
\hline Q. How many Gray rods are needed to fit into the Pink rod? & Q. How many Gray rods are needed to fit into the Pink rod? \\
\hline$P$ & $P$ \\
\hline G & G \\
\hline$\perp$ & \\
\hline 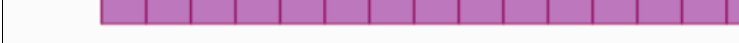 & \\
\hline \begin{tabular}{|l|l|l|l|l|l|l} 
& & & \\
\end{tabular} & \begin{tabular}{|l|l|l|l|l|l|l} 
& & & & \\
\end{tabular} \\
\hline $\begin{array}{l}\qquad[5: 2 \rightarrow 21 / 2] \\
\text { When struggling with the task } 2-1 \text { or } 2-2 \\
\text { (Fixed unit) }\end{array}$ & $\begin{array}{c}{[8: 3 \rightarrow 22 / 3]} \\
\text { (Longer adjustable unit) }\end{array}$ \\
\hline
\end{tabular}

a. How did you get the number of Gray rods?

b. (if a child mentioned the number only (e.g., about three or a half) Three of what? A half of what?

c. (when adjusted the unit of Blue Ruler) How did you decide the size of that unit?

d. (when mentioned a partial unit (e.g., half) with a whole-sized unit of Blue Ruler) Can you show a half with other colored Dynamic Ruler?

e. Why did you put Dynamic Ruler over that place? Is there another way to use the ruler?

f. (Task 3-3) How did you choose the blue (or purple) Dynamic Ruler? 


\section{Appendix B. Initial Coding Scheme}

Initial coding scheme for mathematics words and action by the use of a tool was drawn from literature. The mathematical ideas include "fraction", "unit coordination", "assigning number as measure", "partitioning", "additive reasoning", and "multiplicative reasoning". I will code fractional words such as half and quarter as "fraction". In a length measurement situation, if a student articulates units (e.g., one N, Red segments), a measured result (e.g., four Red segments) and their relationships, those words were categorized as incorporating the ideas of "unit coordination". Specifically, the units might have multiple layers as a composite unit which refers to a containing unit (Steffe, 1992). As the example in Table 1, if a student identified two green segments as a half of the yellow bar $(\mathrm{N})$ then iterated the half as a unit to reproduce the blue bar $(\mathrm{M})$, the student could identify two types of units as Type 2 of unit coordination, employing a composite unit. The units coordination can be identified out of three possible types of units during a series of sessions. When a phrase includes a whole and partitioned parts (e.g., the $\mathrm{N}$ cut into half), such word retained to "partitioning". If a phrase includes additive operations such as addition and subtraction, that will be labeled as "additive" (e.g., "two reds longer"). On the other hand, if it includes skip-counting (e.g., two, four, six), scaling (e.g., times) or multiplicative operations (e.g., multiplication and division), such words will be categorized into "multiplicative". The list of the mathematical ideas was not comprehensive and a word or a phrase can be labeled by different categories.

\section{Table.}

Initial Codes scheme 


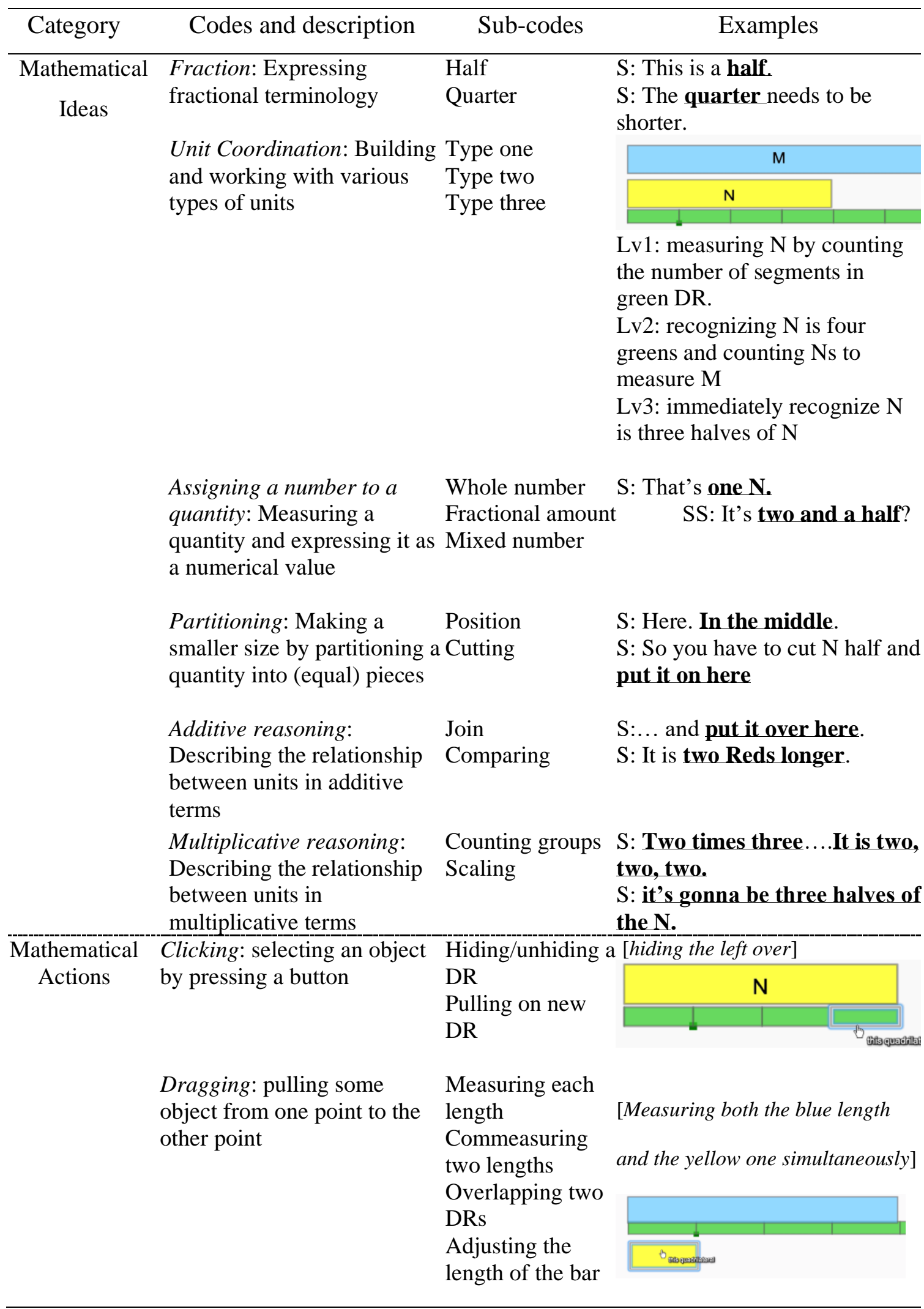




\section{VITA}

Sheunghyun Yeo grew up in Daegu, Korea. He earned a Bachelor of Education degree emphasized on Elementary Mathematics Education from Daegu National University of Education in 2006, a Master of Education in Mathematics Education from Korea National University of Education in 2014. Prior to enrolling in the Ph.D. program at the University of Missouri, Sheunghyun spent ten years in teaching at elementary schools in Deague, Korea. He was awarded a presidential prize in Nationwide Education Materials Exhibition in 2013. Sheunghyun earned a Ph.D. in Learning, Teaching, and Curriculum with a focus on Mathematics Education from the University of Missouri in 2020. He also received a Qualitative Research Graduate Certificate from the University of Missouri in 2020. During the Ph.D. program, Sheunghyun was awarded G. Ellsworth Huggins Graduate Fellowship through the University of Missouri. 